COMMUNICATIONS IN

ANALYSIS AND GEOMETRY

Volume 10, Number 3, 515-573, 2002

\title{
Hopf differentials and the images of harmonic maps
}

\author{
Thomas K.K. AU, LUen-FaI TAM ${ }^{1}$ AND Tom Y.H. WAN ${ }^{2}$
}

In $[\mathrm{Hz}]$, Heinz proved that there is no harmonic diffeomorphism from the unit disk $\mathbb{D}$ onto the complex plane $\mathbb{C}$. The result was generalized by Schoen $[\mathrm{S}]$ and he proved that there is no harmonic diffeomorphism from the unit disk onto a complete surface of nonnegative curvature. Unlike conformal or quasi-conformal maps between Riemann surfaces, the inverse of a harmonic map is not harmonic in general. Hence it is an interesting question whether there is any harmonic diffeomorphism from $\mathbb{C}$ onto $\mathbb{D}$ equipped with the Poincaré metric. In fact a general form of this question was formulated by Schoen $[\mathrm{S}]$ as follows: Is it true that Riemann surfaces which are related by a harmonic diffeomorphism are necessarily quasi-conformally related?

Let us first recall some facts on harmonic maps between surfaces. Let $\Sigma_{1}$ and $\Sigma_{2}$ be two Riemann surfaces with conformal metrics $\rho^{2}(z)|d z|^{2}$ and $\sigma^{2}(h)|d h|^{2}$ respectively. The harmonic map equation for maps from $\Sigma_{1}$ into $\Sigma_{2}$ can be written as

$$
h_{z \bar{z}}+2(\log \sigma)_{h} h_{z} h_{\bar{z}}=0 .
$$

Define $\|\partial h\|=\rho^{-1} \sigma\left|h_{z}\right|$, and $\|\bar{\partial} h\|=\rho^{-1} \sigma\left|h_{\bar{z}}\right|$. Hence $\|\partial h\|$ and $\|\bar{\partial} h\|$ are the norms of the (1,0)-part and (0,1)-part of $d h$. The energy density of $h$ is given by $e(h)=\|\partial h\|^{2}+\|\bar{\partial} h\|^{2}$, and the Jacobian of $h$ is given by $J(h)=$ $\|\partial h\|^{2}-\|\bar{\partial} h\|^{2}$. The Hopf differential of $h$ is defined as $\phi d z^{2}=\sigma^{2}(h) h_{z} \bar{h}_{z} d z^{2}$, which is the $(2,0)$-part of $h^{*}\left(\sigma^{2}(h)|d h|^{2}\right)$. It is well known that if $h$ is harmonic then $\phi d z^{2}$ is a holomorphic quadratic differential defined on $\Sigma_{1}$, see [C-G]. If $h$ is an orientation preserving local diffeomorphism, then $J(u)>0$, which implies that $\|\partial h\|>0$ everywhere, and that

$$
e^{2 w}>|\phi|
$$

where $w=\log \|\partial h\|$.

\footnotetext{
${ }^{1}$ Research was partially supported by a grant from the Research Grants Council of the Hong Kong Special Administrative Region (Project No. CUHK4217/99P)

${ }^{2}$ Research was partially supported by a grant from the Research Grants Council of the Hong Kong Special Administrative Region (Project No. CUHK4199/97P)
} 
In [Wn], the third author proved that orientation preserving harmonic diffeomorphisms on the hyperbolic plane $\mathbb{H}^{2}$ can be parametrized by their Hopf differentials, provided that they can be realized as the Gauss maps of constant mean curvature cuts in the Minkowski space $M^{2,1}$. The last condition is equivalent to the fact that $|\partial h|^{2}|d z|^{2}$ is a complete metric on $\mathbb{D}$, where $|\partial h|$ is the norm of $\partial h$ with respect to the Euclidean metric on the domain and the Poincare metric on the target. The result was generalized to harmonic maps from $\mathbb{C}$ into $\mathbb{H}^{2}$ in $[\mathrm{W}-\mathrm{A}]$ and to more general surfaces in [T-W1].

Hence in order to study the behaviors of harmonic maps from $\mathbb{C}$ or $\mathbb{D}$ into $\mathbb{H}^{2}$ it is useful to study their Hopf differentials. In [Hn], Han proved that if $h$ is a harmonic diffeomorphism from $\mathbb{C}$ into $\mathbb{H}^{2}$ whose Hopf differential is a polynomial, then the closure of $h(\mathbb{C})$ in $\overline{\mathbb{H}^{2}}=\mathbb{H}^{2} \cup \partial \mathbb{H}^{2}$ is the convex hull of a totally disconnected closed set in $\partial \mathbb{H}^{2}$, provided that $|\partial h|^{2}|d z|^{2}$ is complete on $\mathbb{C}$. In particular, $h$ is not surjective. Here $\partial \mathbb{H}^{2}$ is the geometric boundary of $\mathbb{H}^{2}$. Later in [HTTW], it was proved that the closure of the image of an orientation preserving harmonic diffeomorphism $h$ from $\mathbb{C}$ into $\mathbb{H}^{2}$ is an ideal polygon with exactly $m+2$ vertices on $\partial \mathbb{H}^{2}$ if and only if the Hopf differential $\phi d z^{2}$ is a polynomial of degree $m$, i.e. $\phi$ is a polynomial of degree $m$. Note that by [Wn, T-W1], we know that $\phi$ is of degree no greater than $m$ if and only if $h$ is of polynomial growth of degree at most $m / 2+1$.

In higher dimensions, one cannot expect that such a clean statement continues to hold. However, in [L-W1], Li and Wang were able to generalized part of the above result for a much more general class of manifolds. They proved that if $M^{n}$ is a complete manifold with nonnegative Ricci curvature and $N$ is a Cartan-Hadamard manifold with sectional curvature pinched between two negative constants, then the closure in $N \cup \partial N$ of the image of a harmonic map from $M^{n}$ into $N$ with polynomial growth of degree at most $\ell$ is in the convex hull of finitely many points on the geometric boundary $\partial N$ of $N$. Moreover, the number of points is bounded by a constant depending only on $n$ and $\ell$. Actually, they only assumed that $M$ satisfies the so-called weak volume growth condition and weak Poincaré inequality. In [L-W3], they also obtained a sharp estimate for the number of points on the ideal boundary in case $M$ is a complete surface with finite total curvature.

All these results in [Hn, HTTW, L-W1, L-W3] are under the assumption that the harmonic map is of polynomial growth. In this paper, we want to study harmonic maps from $\mathbb{C}$ into $\mathbb{H}^{2}$ which grow faster than polynomial. We will study the images of the harmonic maps by a careful study of their Hopf differentials.

First we prove that if $h$ is an orientation preserving harmonic diffeomor- 
phism with Hopf differential $\phi d z^{2}$ such that $\phi$ is of one of the following forms then $h$ is not surjective:

(1) $\phi=P_{1} \exp \left[P_{2} \exp \left[\cdots \exp \left[P_{k} \exp (Q)\right] \cdots\right]\right]$, where $P_{j}$ and $Q$ are polynomials (Theorem 1.2);

(2) $\phi=P \exp (Q)$ where $Q(z)=z^{n}+\sum_{j=1}^{n} a_{j} z^{n-j}$ is a polynomial of degree $n \geq 1, P$ is entire with order $\rho<n$ and

$$
\Sigma \cap\left\{z|| z \mid>R_{0} \text { and }-\delta<\arg z<\delta\right\}=\emptyset,
$$

for some $\frac{\pi}{n}>\delta>0$ and $R_{0}>0$, where $\Sigma$ is the set of all zeros of $P$ (Theorem 1.1);

(3) $\phi=\left(f^{\prime}\right)^{2}$ where $f$ is entire with no finite asymptotic value in the domain

$$
\mathcal{R}=\left\{z \mid \frac{\pi}{2}-\delta<\arg z<\frac{\pi}{2}+\delta, \text { and }|z|>R\right\}
$$

for some $\delta>0$ and $R>0$ and $f^{\prime}(z) \neq 0$ for all $z$ in $f^{-1}(\mathcal{R})$ (Theorem $1.3)$.

In (2), $\phi$ is of finite order, in (1) $\phi$ is of infinite order and there is no growth condition in (3). Note that if $\phi$ is of finite order then $\phi=P \exp (Q)$ with $Q$ being a polynomial and $P$ is entire.

As mentioned above, if $|\partial h|^{2}|d z|^{2}$ is complete on $\mathbb{C}$ and if $\phi$ is a polynomial, then the image of $h$ is an ideal polygon with finitely many vertices at $\partial \mathbb{H}^{2}$ [HTTW]. If $\phi$ is not a polynomial, then $\overline{h(\mathbb{C})} \cap \partial \mathbb{H}^{2}$ must consist of infinitely many points by [HTTW] again and in this case, the image set is much more difficult to be described. In the second part of this paper, we want to describe the images of harmonic maps under similar assumptions as in (1) or (2) above. We prove that if $\phi=P \exp (Q)$ where $P$ and $Q$ are polynomials, then $\overline{h(\mathbb{C})} \cap \partial \mathbb{H}^{2}$ is countable and consists of exactly $n$ accumulation points, where $n=\operatorname{deg} Q$ (Theorem 3.1). In fact, one can relax the condition that $P$ is polynomial. If we assume that $P$ is entire with order less than $n$ and the zeros of $P$ are well distributed, then the same conclusion holds. Next we consider the case that $\phi(z)=P\left(e^{z}\right)$ where $P(t)=\sum_{k=-m}^{n} a_{k} t^{k}$ and is non-constant. In this case $\phi$ is of order one. It is interesting to know that under this assumption on $\phi, \overline{h(\mathbb{C})} \cap \partial \mathbb{H}^{2}$ has only one accumulation points in some cases and has exactly two accumulation points in other cases (Theorem 4.1). In case $\phi$ has infinite order, then the image set is even more complicated. We are able to prove that if $\phi(z)=\exp ^{(k)}(z) d z^{2}$, for some 
positive integer $k$, where $\exp ^{(k)}(z)$ is defined inductively by $\exp ^{(0)}(z)=1$ and $\exp ^{(j)}(z)=\exp \left(\exp ^{(j-1)}(z)\right)$, then $\overline{h(\mathbb{C})} \cap \partial \mathbb{H}^{2}=\cup_{j=0}^{k} \mathcal{A}_{j}$ such that $A_{j}$ is countable and discrete for each $0 \leq j \leq k-1, \mathcal{A}_{j}$ consists of all isolated accumulation points of $\mathcal{A}_{j-1}$ for $1 \leq j \leq k$, and $\mathcal{A}_{k}$ consists of only one point (Theorem 4.2).

In order to prove these results, we have to study the regions where $|\phi|$ grows very fast and the regions where $|\phi|$ decays or is bounded. In the regions where $|\phi|$ grows very fast, we refine the technique in [Hn, HTTW] which was introduced by Wolf and Minsky [Wf, M]. In order to study the regions where $\phi$ is bounded, we need other tools. We will use the idea of the so-called maximal $\Phi$-radius of a holomorphic quadratic differential $\Phi$, see $\S 2$ for definitions. Let $\Phi$ be a holomorphic quadratic differential on $\mathbb{D}$. It was proved by Anić, Marković and Mateljević [A-M-M] that the norm of $\Phi$ with respect to the Poincaré metric is uniformly bounded if and only if the maximal $\Phi$-radius is uniformly bounded. On the other hand, it was proved in [Wn] that $h$ is a quasi-conformal harmonic diffeomorphism from $\mathbb{H}^{2}$ onto itself if and only if the norm of its Hopf differential is uniformally bounded. Hence we can conclude that $h$ is quasi-conformal if and only if the maximal $\Phi$-radius is uniformly bounded where $\Phi$ is the Hopf differential of $h$. In fact, it was proved in [A-M-M] that if $h$ is quasi-regular harmonic map on $\mathbb{H}^{2}$ then the maximal $\Phi$-radius is uniformly bounded. In this work, we will give a local version of these results. In Theorem 2.1, we will prove that if $h$ is an orientation preserving harmonic diffeomorphism from $\mathbb{D}$ or $\mathbb{C}$ into $\mathbb{H}^{2}$, under certain conditions, $h$ is quasi-conformal on the domains where the maximal $\Phi$-radius is uniformly bounded, where $\Phi$ is the Hopf differential of $h$. In particular, we give another proof of the result in [Wn] mentioned above. Roughly speaking, in the case of harmonic diffeomorphisms from $\mathbb{C}$ into $\mathbb{H}^{2}$, the domains where the $\Phi$-radius is uniformly bounded are the domains where $|\phi|$ is uniformly bounded and decays rapidly at infinity.

In the process of proving Theorem 2.1, we need a refined version of the result in $[\mathrm{A}-\mathrm{M}-\mathrm{M}]$ on the relation between the maximal $\Phi$-radius and the norm of the holomorphic quadratic differential $\Phi$. In particular, we obtain a pointwise lower bound of the maximal $\Phi$-radius (Proposition 2.1). It turns out that the result also has applications to the problem of finding quasiconformal harmonic diffeomorphism on $\mathbb{H}^{2}$ with prescribed quasi-symmetric function on the unit circle $\mathbb{S}^{1}$ which is identified as $\partial \mathbb{H}^{2}$. Let $\operatorname{BQD}\left(\mathbb{H}^{2}\right)$ be the space of holomorphic quadratic differentials $\Phi$ on $\mathbb{H}^{2}$ such that

$$
\||\Phi|\|=\sup _{z \in \mathbb{H}^{2}}\|\Phi\|(z)<\infty
$$


where $\|\Phi\|(z)$ is the norm of $\Phi$ at $z$ with respect to the Poincare metric. In [Wn], a map $\mathfrak{B}$ from $\mathrm{BQD}\left(\mathbb{H}^{2}\right)$ to the universal Teichmüller space $\mathcal{T}$ by sending $\Phi$ to the class of quasi-symmetric homeomorphism containing the boundary value of $h$, which is the quasi-conformal harmonic diffeomorphism on $\mathbb{H}^{2}$ with $\Phi$ as the Hopf differential. The map is injective [L-T3, L-W2] and an open question is whether this map is surjective. This is in fact a conjecture of Schoen [S]. There are partial results for this problem as well as similar problems in higher dimensions [Ak, L-T1, L-T2, L-T3, T-W2, T-W3, $\mathrm{H}-\mathrm{W}, \mathrm{Y}, \mathrm{S}-\mathrm{T}-\mathrm{W}]$. In our case, it is not hard to see that if one can prove that $\mathfrak{B}$ is 'proper', namely, the inverse image of bounded set is bounded, then one can conclude that $\mathfrak{B}$ is onto. Using the pointwise estimate of the maximal $\Phi$-radius and the main inequality of Reich and Strebel [R-S], we obtain sufficient conditions for certain subspaces of $\mathrm{BQD}\left(\mathbb{H}^{2}\right)$ on which $\mathfrak{B}$ is proper. For compact Riemann surfaces or Riemann surfaces of finite type, this kind of phenomena was studied by Wolf [Wf] and Marković-Mateljević $[M-M]$. In $[M-M]$, a generalized version of the inequality in [R-S] was used.

We organize the paper as follows. In $\S 1$, we discuss some non-surjectivity results of harmonic maps from $\mathbb{C}$ into $\mathbb{H}^{2}$. In $\S 2$, we study the relation between maximal $\Phi$-radius of the Hopf differential $\Phi$ of a harmonic map and quasi-conformality. In $\S 3$ and $\S 4$, we study the structures of images of harmonic maps from $\mathbb{C}$ into $\mathbb{H}^{2}$. In $\S 5$, we use the result in $\S 2$ to study quasi-conformal harmonic diffeomorphisms on $\mathbb{H}^{2}$. In the appendix, we use Mathematica to produce figures of horizontal trajectories defined by different types of holomorphic quadratic differentials discussed in this work, so that one may get some feelings about the images of related harmonic maps.

Finally, the authors would like to thank the referee for pointing out a gap in the proof of theorem 1.1, which has been corrected accordingly.

\section{Results on non-surjectivity of harmonic diffeomorphisms.}

In [HTTW], it was proved that a polynomial growth harmonic diffeomorphism from $\mathbb{C}$ into $\mathbb{H}^{2}$ is not surjective. In [L-W1], the result was generalized to higher dimensions for polynomial growth harmonic maps between a more general class of manifolds. Not very many results are known if the map grows faster than polynomial. In this section, we will give results on nonsurjectivity of certain harmonic diffeomorphisms from $\mathbb{C}$ into $\mathbb{H}^{2}$ with fast growth rate. Note that the growth rate of a harmonic diffeomorphism from $\mathbb{C}$ into $\mathbb{H}^{2}$ can be expressed in terms of the growth rate of its Hopf differential, see [T-W1]. In particular, such a map is of polynomial growth if and 
only if its Hopf differential is of the form $P d z^{2}$ with $P$ to be a polynomial.

Lemma 1.1. Let $\Omega$ be a domain in $\mathbb{C}$ which contains every disk $\mathbb{D}(\sqrt{-1} y, R(y))$ with center $\sqrt{-1} y$ and radius $R(y) \geq 2 \sqrt{2}(1+\epsilon) \log y$ for all $y \geq y_{0}>0$, where $y_{0}>0, \epsilon>0$ are constants. Suppose $h$ is an orientation preserving harmonic diffeomorphism from $\Omega$ into $\mathbb{H}^{2}$ with Hopf differential $\Phi=d z^{2}$. Then the length of the image of the half line $\Im z \geq y_{0}, \Re z=0$ under $h$ is bounded by a constant depending only on $\epsilon$ and $y_{0}$.

Proof. Let $\exp (w)=\|\partial h\|$ be the norm of $\partial h$ and let $e$ be the energy density of $h$ with respect to the Euclidean metric in the domain, then the pull-back metric under $h$ is given by

$$
\begin{aligned}
h^{*}\left(d s_{\mathbb{H}^{2}}^{2}\right) & =(e+2) d x^{2}+(e-2) d y^{2} \\
& =2(\cosh (2 w)+1) d x^{2}+2(\cosh (2 w)-1) d y^{2} .
\end{aligned}
$$

As in [Wf, M] and p. 63 in [Hn] we can prove that there is $y_{0}>0$ such that if $y \geq y_{0}$

$$
0<w(\sqrt{-1} y) \leq C_{1} \exp \left(-\frac{R(y)}{2 \sqrt{2}}\right)
$$

where $C_{1}$ is an absolute constant. Hence the length $\ell$ of the image of $\{\Im z \geq$ $\left.y_{0}, \Re z=0\right\}$ under $h$ satisfies:

$$
\begin{aligned}
\ell & =\int_{y_{0}}^{\infty}[2(\cosh (2 w)-1)]^{\frac{1}{2}} d y \\
& \leq C_{3} \int_{y_{0}}^{\infty} \exp \left(-\frac{R(y)}{2 \sqrt{2}}\right) d y \\
& \leq C_{4} \int_{y_{0}}^{\infty} y^{-1-\epsilon} d y \\
& =C_{5}
\end{aligned}
$$

where $C_{3}-C_{5}$ are constants depending only on $\epsilon$ and $y_{0}$, and we have used (1.1), (1.2) and the assumption that $R(y) \geq 2 \sqrt{2}(1+\epsilon) \log y$ if $y \geq y_{0}$. The lemma then follows.

The following lemma basically says that if $Q(z)=\frac{1}{2} z+o(1)$ as $\Re z \rightarrow \infty$, then the behavior of $\int \exp (Q(z)) d z$ is similar to that of $\int \exp \left(\frac{1}{2} z\right) d z$. 
Lemma 1.2. Let $2 \pi \geq A>0$ and let $Q(z)$ be an analytic function on the half strip

$$
\mathcal{S}=\{z \mid \Re z>\alpha>0 \text { and } \theta-A<\Im z<\theta+A\}
$$

where $\theta$ is a constant. Suppose $Q(z)=\frac{1}{2} z+q(z)$ such that $|q(z)| \leq g(\Re z)$ where $g(t) \geq 0$ is a function defined on $\infty>t \geq \alpha$ which satsifies $\lim _{t \rightarrow \infty} g(t)=0$. Then for any $\frac{1}{4} A>\delta>0$, there exists $\mathfrak{a}>0$ depending only on $A, \delta, \alpha$ and the function $g$ such that if $z_{0}=x_{0}+\sqrt{-1} \theta$ with $x_{0}>\mathfrak{a}$ and if

$$
\zeta(z)=\int_{z_{0}}^{z} \exp (Q(\xi)) d \xi
$$

then $\zeta$ maps $\mathcal{S}_{\delta}$ injectively into $\zeta$-plane, and $\zeta\left(\mathcal{S}_{\delta}\right) \supset \mathcal{R}_{2 \delta} \supset \zeta\left(\mathcal{S}_{4 \delta}\right)$. Here

$$
\mathcal{S}_{\delta}=\left\{z \in \mathcal{S} \mid \Re z>x_{0}+\delta, \theta-A+\delta<\Im z<\theta+A-\delta\right\},
$$

$\mathcal{S}_{4 \delta}$ is defined similarly and

$$
\begin{gathered}
\mathcal{R}_{2 \delta}=-2 \exp \left(\frac{1}{2} z_{0}\right)+\left\{\zeta|| \zeta \mid>2 \exp \left(\frac{1}{2}\left(x_{0}+2 \delta\right)\right),\right. \\
\left.\frac{1}{2}(\theta-A+2 \delta)<\arg \zeta<\frac{1}{2}(\theta+A-2 \delta)\right\} .
\end{gathered}
$$

Proof. Since $\lim _{t \rightarrow \infty} g(t)=0$, for any $\epsilon>0$, there is $\mathfrak{a}>\alpha$ depending only on $\alpha$ and $g$ such that if $\Re z>\mathfrak{a}$, then

$$
\left|\exp (Q(z))-\exp \left(\frac{z}{2}\right)\right| \leq \epsilon \exp \left(\frac{1}{2} \Re z\right) .
$$

Let $x_{0}>\mathfrak{a}$ and let $f(z)=2\left(\exp \left(\frac{1}{2} z\right)-\exp \left(\frac{1}{2} z_{0}\right)\right)$ with $z_{0}=x_{0}+i \theta$. Let $z_{1}=x_{1}+\sqrt{-1} y_{1}, z_{2}=x_{2}+\sqrt{-1} y_{2}$ in $\mathcal{S}$ such that $x_{1}, x_{2}$ are larger than $x_{0}$. Suppose $x_{1}>x_{2}$, then by (1.3)

$$
\begin{aligned}
& \left|\zeta\left(z_{1}\right)-\zeta\left(z_{2}\right)-f\left(z_{1}\right)+f\left(z_{2}\right)\right| \\
& =\left|z_{1}-z_{2}\right|\left|\int_{0}^{1}\left(\exp \left(Q\left(t z_{1}+(1-t) z_{2}\right)\right)-\exp \left(\frac{1}{2}\left(t z_{1}+(1-t) z_{2}\right)\right)\right) d t\right| \\
& \leq \epsilon\left(\left|x_{1}-x_{2}\right|+4 \pi\right) \exp \left(\frac{1}{2} x_{2}\right) \int_{0}^{1} \exp \left(\frac{t}{2}\left(x_{1}-x_{2}\right)\right) d t \\
& \leq \epsilon \exp \left(\frac{1}{2} x_{2}\right)\left[2\left(\exp \left(\frac{1}{2}\left(x_{1}-x_{2}\right)\right)-1\right)+4 \pi \exp \left(\frac{1}{2}\left(x_{1}-x_{2}\right)\right)\right] \\
& \leq C_{1} \epsilon \exp \left(\frac{1}{2} x_{1}\right)
\end{aligned}
$$


where $C_{1}$ is an absolute constant. Obviously, the inequality is still true if $x_{1}=x_{2}$. Hence, we have

$$
\left|\zeta\left(z_{1}\right)-\zeta\left(z_{2}\right)-f\left(z_{1}\right)+f\left(z_{2}\right)\right| \leq C_{1} \epsilon \exp \left(\frac{1}{2} \max \left\{\Re z_{1}, \Re z_{2}\right\}\right)
$$

provided $\Re z_{1}, \Re z_{2}>x_{0}$.

On the other hand, for any $0<\delta_{1}<A$ and $z_{1} \neq z_{2} \in \mathcal{S}$, with $\Re z_{1} \geq \Re z_{2}$ and $\left|\Im\left(z_{1}-z_{2}\right)\right| \leq 2 A-2 \delta_{1}$, we have

$$
\begin{aligned}
\left|f\left(z_{1}\right)-f\left(z_{2}\right)\right| & =2 \exp \left(\frac{1}{2} \Re z_{1}\right)\left|1-\exp \left(\frac{1}{2}\left(z_{2}-z_{1}\right)\right)\right| \\
& \geq \tau \exp \left(\frac{1}{2} \Re z_{1}\right)
\end{aligned}
$$

where $\tau>0$ depends only on $A-\delta_{1}$ and the lower bound of $\left|z_{1}-z_{2}\right|$ where we have used the fact that $\left|\Im\left(z_{1}-z_{2}\right)\right| \leq 2 A-2 \delta_{1} \leq 4 \pi-2 \delta_{1}$. Hence for any $z_{1} \neq z_{2} \in \mathcal{S}$,

$$
\left|f\left(z_{1}\right)-f\left(z_{2}\right)\right| \geq \tau \exp \left(\frac{1}{2} \max \left\{\Re z_{1}, \Re z_{2}\right\}\right)
$$

where $\tau>0$ depending only on the lower bound of $\left|z_{1}-z_{2}\right|$.

Let $0<\delta_{1}<A$, for any $a$ in $\mathcal{S}_{\delta_{1}} \cap\{z \mid \Re z<\beta\}$ and $z$ on the boundary of $\mathcal{S}_{\frac{1}{2} \delta_{1}} \cap\left\{z \mid \Re z<\beta+\frac{1}{2} \delta_{1}\right\}$ where $\beta$ is a large number, we have

$$
\begin{aligned}
|f(z)-f(a)| & \geq \tau \exp \left(\frac{1}{2} \Re z\right) \\
& \geq \frac{\tau}{C_{1} \epsilon}|\zeta(z)-f(z)|
\end{aligned}
$$

by (1.4) with $z_{1}=z$ and $z_{2}=z_{0}$, and (1.5), where $\tau>0$ is a constant depending only on $\delta_{1}$. Here we take $z_{1}=z$ and $z_{2}=z_{0}$ in (1.4). Choose $\epsilon$ small enough depending only on $A$ and $\delta_{1}$ such that $\frac{\tau}{C_{1} \epsilon}>1$, we have

$$
|f(z)-f(a)|>|\zeta(z)-f(z)| \text {. }
$$

Apply the Rouché Theorem to the functions $\zeta-f(a), f-f(a)$ on $\mathcal{S}_{\frac{1}{2} \delta_{1}} \cap$ $\left\{z \mid \Re z<\beta+\frac{1}{2} \delta_{1}\right\}$ and then let $\beta \rightarrow \infty$ we conclude that for any $a \in \mathcal{S}_{\delta_{1}}$ there is one and only one $z \in S_{\frac{1}{2} \delta_{1}}$ such that $\zeta(z)=f(a)$.

On the other hand for such an $a$, we have

$$
\begin{aligned}
|\zeta(z)-\zeta(a)| & \geq|f(z)-f(a)|-|\zeta(z)-\zeta(a)-f(z)+f(a)| \\
& \geq \frac{1}{2}|f(z)-f(a)| \\
& >|\zeta(z)-f(z)|
\end{aligned}
$$


provided $\epsilon$ is chosen to be small enough (depending only on $A$ and $\delta_{1}$ ). Hence there is also exactly one $z \in S_{\frac{1}{2} \delta_{1}}$ such that $f(z)=\zeta(a)$. From these the lemma follows by considering the image of $f$.

In the next theorem we will study the surjectivity of those harmonic diffeomorphisms from $\mathbb{C}$ into $\mathbb{H}^{2}$ whose Hopf differentials are of finite order.

Theorem 1.1. Let $h$ be an orientation preserving harmonic diffeomorphism from $\mathbb{C}$ into $\mathbb{H}^{2}$ with Hopf differential $\Phi=P \exp (Q) d z^{2}$ such that

(i) $Q(z)=z^{n}+\sum_{j=1}^{n} a_{j} z^{n-j}$ is a polynomial of degree $n \geq 1$;

(ii) $P$ is entire with order $\rho<n$;

(iii) there exists $\frac{\pi}{n}>\delta>0$ and $R_{0}>0$ such that

$$
\Sigma \cap\left\{z|| z \mid>R_{0} \text { and }-\delta<\arg z<\delta\right\}=\emptyset,
$$

where $\Sigma$ is the set of all zeros of $P$.

Then $h$ is not surjective. In particular, if $P$ is a polynomial then $h$ is not surjective.

Proof. By the Hadamard factorization theorem, $P(z)=z^{m} e^{a(z)} A(z)$, where $m$ is the order of zero of $P$ at $z=0, a(z)$ is a polynomial of degree less than $\rho<n$, and $A(z)$ is a canonical product of order less than or equal to $\rho$ formed by the zeros of $P$. So we can absorb $a(z)$ to the lower order terms of $Q(z)$ and assume $P(z)$ has the form $z^{m} A(z)$.

Let $\zeta_{1}=z^{n}$, which will map $-\delta<\arg z<\delta$ bijectively onto $-n \delta<$ $\arg \zeta_{1}<n \delta$. In the region

$$
\begin{gathered}
\mathcal{R}_{1}=\left\{\left|\zeta_{1}\right|>R_{0}^{n}\right\} \cap\left\{-n \delta<\arg \zeta_{1}<n \delta\right\} . \\
\Phi=n^{-2} \zeta_{1}^{-\frac{2(n-1)}{n}} \tilde{P}\left(\zeta_{1}\right) \exp \left(\zeta_{1}\right) d \zeta_{1}^{2}
\end{gathered}
$$

where $\tilde{P}\left(\zeta_{1}\right)=P\left(z\left(\zeta_{1}\right)\right) \exp \left(\sum_{j=1}^{n} a_{j} \zeta_{1}^{1-j / n}\right)$. By (ii), without loss of generality we may assume that for $\left|\zeta_{1}\right|>R_{0}^{n},\left|\tilde{P}\left(\zeta_{1}\right)\right| \leq \exp \left(\left|\zeta_{1}\right|^{\epsilon}\right)$ for some $\epsilon>0$ which is small enough such that $\epsilon<1$. By (iii) and lemma 2.6.18 in [B], we have for any $\eta>0, \log |A(z)|>-|z|^{\rho+\eta}$ on $\left\{z|| z \mid>R_{0}\right.$ and $-\delta<$ $\arg z<\delta\}$ for a possibly larger $R_{0}$ and a smaller $\delta$. Therefore, we have $|\log | \tilde{P}\left(\zeta_{1}\right) \mid=O\left(\left|\zeta_{1}\right|^{\epsilon}\right)$ as $\zeta_{1} \rightarrow \infty$ for some $\epsilon<1$. From this, we have 
$|\nabla \log | \tilde{P}\left(\zeta_{1}\right)||=o(1)$ on $\tilde{\mathcal{R}}_{1}=\mathcal{R}_{1} \cap\left\{-n \delta+\delta_{1}<\arg \zeta_{1}<n \delta-\delta_{1}\right\}$ for any $\delta_{1}>0$; and hence $\left|\frac{d}{d \zeta_{1}} \log \tilde{P}\right|=o(1)$ as $\zeta_{1} \rightarrow \infty$ and $\zeta_{1} \in \tilde{\mathcal{R}}_{1}$. We conclude that in $\tilde{\mathcal{R}}_{1}$,

$$
\Phi=\exp \left(Q_{1}\left(\zeta_{1}\right)\right) d \zeta_{1}^{2}
$$

where

$$
Q_{1}\left(\zeta_{1}\right)=\zeta_{1}+Q_{2}\left(\zeta_{1}\right)
$$

with $-n \delta+\delta_{1}<\arg \zeta_{1}<n \delta-\delta_{1}$ and $\left|\zeta_{1}\right|>R_{0}^{n}$. Here $Q_{2}\left(\zeta_{1}\right)=o\left(\left|\zeta_{1}\right|\right)$ and $\frac{d}{d \zeta_{1}} Q_{2}\left(\zeta_{1}\right)=o(1)$ as $\zeta_{1} \rightarrow \infty$.

Let $\zeta_{2}=\zeta_{1}+Q_{2}\left(\zeta_{1}\right)$. This will map $\left\{-\pi<\arg \zeta_{1}<\pi\right\} \cap\left\{\left|\zeta_{1}\right|>R_{1}\right\}$ injectively onto its image for some $R_{1}>R_{0}^{n}$. Moreover, there exists $R_{2}>0$ such that $\mathcal{R}_{2}=\left\{-\pi+2 \delta_{1}<\arg \zeta_{2}<\pi-2 \delta_{1}\right\} \cap\left\{\left|\zeta_{2}\right|>R_{2}\right\}$ is in the image of the map $\zeta_{1} \mapsto \zeta_{2}$. In $\mathcal{R}_{2}, \Phi$ can be written in the form

$$
\Phi=\left[1+\frac{d Q_{2}}{d \zeta_{1}}\right]^{-2} \exp \left(\zeta_{2}\right) d \zeta_{2}^{2}=\exp \left(Q_{3}\left(\zeta_{2}\right)\right) d \zeta_{2}^{2}
$$

where $Q_{3}\left(\zeta_{2}\right)=\zeta_{2}+o(1)$ as $\left|\zeta_{2}\right| \rightarrow \infty$. Let $\zeta\left(\zeta_{2}\right)=\int^{\zeta_{2}} \exp \left(Q_{3}(\xi)\right) d \xi$. By Lemma 1.2 , we conclude that $\zeta\left(\zeta_{2}\right)$ will map a subdomain of $\mathcal{R}_{2}$ bijectively onto the region

$$
\mathcal{R}=\left\{\zeta|| \zeta \mid>R \text { and } \frac{1}{2} \pi-\frac{1}{2} \delta_{2}<\arg \zeta<\frac{1}{2} \pi+\frac{1}{2} \delta_{2}\right\}
$$

for some $R>0$ and $\delta_{2}>0$. On $\mathcal{R}, \Phi=d \zeta^{2}$. The map $\zeta \mapsto \zeta_{2} \mapsto \zeta_{1} \mapsto z$ is injective when restricted on $\mathcal{R}$. Hence $h(z(\zeta))$ is an orientation harmonic diffeomorphism from $\mathcal{R}$ into $\mathbb{H}^{2}$. By Lemma 1.1, we conclude that the length of the image of the half line $\Im \zeta>a_{0}, \Re \zeta=0$ under $h$ is finite. Here $a_{0}$ is a large constant. By the definition of $\zeta, \Im \zeta \rightarrow \infty$ with $\Re \zeta=0$ implies that $z \rightarrow \infty$. Hence $h$ cannot be surjective.

Please see the appendix for figures showing the behaviour of the horizontal trajectories for some typical examples of holomorphic quadratic differentials discussed here (figures 1-6). If we refine the method of proof in Theorem 1.1, we can generalize the result to some cases that the Hopf differentials grow very fast (see figure 7 in appendix). First we have the following:

Lemma 1.3. Let $R_{0}>0$ and $\delta>0$ be constants and let $h$ be an orientaion preserving harmonic diffeomorphism from $\Omega_{\delta}=\left\{|z|>R_{0},|\arg z|<\pi-\delta\right\}$ into $\mathbb{H}^{2}$ with Hopf differential of the form

$$
\Phi=\exp \left[g_{1}+\exp \left[g_{2}+\cdots+\exp \left[g_{k}+Q\right] \cdots\right]\right] d z^{2}
$$


where $Q(z)=z^{n}+\sum_{j=1}^{n} a_{j} z^{n-j}$ is a polynomial in $z$ and for each $j=$ $1, \ldots, k,\left|g_{j}(z)\right|=O(\log |z|)$ as $|z| \rightarrow \infty$. Then there exists a path in $\Omega_{\delta}$ diverging to infinity such that its image under $h$ has finite length.

Proof. We will prove by induction on $k$. For $k=1, \Phi=\exp \left[g_{1}+Q\right] d z^{2}$ and we can apply the same proof as in Theorem 1.1 to conclude the existence of such path.

For $k \geq 2$, we consider the $\operatorname{map} \zeta=f(z)=Q(z)+g_{k}(z)$ on a convex subdomain $\mathcal{R}$ in $\Omega_{\delta}$ defined by

$$
\mathcal{R}=\left\{z \in \Omega_{\delta} \mid \Re z \geq R_{1},-\frac{\pi}{2(n-1)}+\epsilon<\arg z<\frac{\pi}{2(n-1)}-\epsilon\right\}
$$

where $R_{1}>R_{0}$ and $\epsilon>0$ will be chosen later. It is clear that for $z=r e^{i \theta} \epsilon$ $\mathcal{R}$ with $r$ sufficiently large,

$$
\Re f^{\prime}(z)=n r^{n-1} \cos [(n-1) \theta]+o\left(r^{n-1}\right)>0 .
$$

Therefore, if we choose $R_{1}$ sufficiently large, $\zeta=f(z)$ maps $\mathcal{R}$ one-one onto its image $f(\mathcal{R})$ as $\mathcal{R}$ is convex (Proposition 1.10 in [P]). Since

$$
f\left(r e^{ \pm i\left(\frac{\pi}{2(n-1)}-\epsilon\right)}\right)=r^{n} e^{ \pm i\left(n \frac{\pi}{2(n-1)}-n \epsilon\right)}+o\left(r^{n}\right),
$$

it is clear that $f(\mathcal{R})$ contains a subset of the form

$$
\left\{|\arg \zeta|<n \pi / 2(n-1)-\epsilon_{1},|\zeta|>R_{2}\right\} .
$$

If we choose $\epsilon<\frac{\pi}{2 n(n-1)}$, then we can choose accordingly an $\epsilon_{1}$ such that $\frac{n \pi}{2(n-1)}-\epsilon_{1}>\pi / 2$. Hence, under this choice of $R_{1}$ and $\epsilon, f(\mathcal{R})$ contains a half-plane $\left\{\Re \zeta>R_{2}\right\}$ for some $R_{2}>0$.

On $f(\mathcal{R})$, in particular on the half-plane $\left\{\Re \zeta>R_{2}\right\}, \Phi$ can be written as

$$
\Phi=\exp \left[\tilde{g}_{1}(\zeta)+\exp \left[\tilde{g}_{2}(\zeta)+\cdots+\exp \left[\tilde{g}_{k-1}(\zeta)+\exp \zeta\right] \cdots\right]\right] d \zeta^{2},
$$

where $\tilde{g}_{1}(\zeta)=g_{1}\left(f^{-1}(\zeta)\right)-2\left(\frac{d}{d z} \log f\right)\left(f^{-1}(\log \xi)\right)$ and $\tilde{g}_{j}(\zeta)=g_{j}\left(f^{-1}(\zeta)\right)$ for $j=2, \ldots, k-1$.

Now, for any small $\delta_{1}>0$, let us consider the half strip

$$
\mathcal{S}=\left\{\Re \zeta>R_{2},|\Im \zeta|<\pi-\delta_{1}\right\} .
$$


The exponential map $\xi=\exp \zeta$ maps $\mathcal{S}$ one-one onto the domain

$$
\Omega_{\delta_{1}}=\left\{|\xi|>R_{3},|\arg \xi|<\pi-\delta_{1}\right\},
$$

where $R_{3}=e^{R_{2}}$. And on $\Omega_{\delta_{1}}, \Phi$ takes the form

$$
\Phi=\exp \left[\tilde{\tilde{g}}_{1}(\xi)+\exp \left[\tilde{\tilde{g}}_{2}(\xi)+\cdots+\exp \left[\tilde{\tilde{g}}_{k-1}(\xi)+\xi\right] \cdots\right]\right] d \xi^{2},
$$

where

$$
\tilde{\tilde{g}}_{1}(\xi)=g_{1}\left(f^{-1}(\log \xi)\right)-2\left(\frac{d}{d z} \log f\right)\left(f^{-1}(\log \xi)\right)-2 \log \xi
$$

and $\tilde{\tilde{g}}_{j}(\xi)=g_{j}\left(f^{-1}(\log \xi)\right)$ for $j=2, \ldots, k-1$.

Since $\left|g_{j}(z)\right|=O(\log |z|)$ and $\zeta=f(z) \sim z^{n}$ as $|z| \rightarrow \infty$, we see that

$$
\left|g_{j}\left(f^{-1}(\log \xi)\right)\right|=O(\log \log |\xi|) \quad \text { for } j=1, \ldots, k-1 .
$$

We also conclude from $\frac{d f}{d z}(z) \sim z^{n-1} \sim \zeta^{(n-1) / n}$ as $|\zeta| \rightarrow \infty$ that

$$
\left|\left(\frac{d}{d z} \log f\right)\left(f^{-1}(\log \xi)\right)\right|=O(\log \log |\xi|) .
$$

All together we have, as $|\xi| \rightarrow \infty$,

$$
\left|\tilde{\tilde{g}}_{j}(\xi)\right|=\tilde{O}(\log |\xi|) .
$$

Therefore, induction hypothesis implies that there exists a divergent path $\xi=\gamma(t)$ in $\Omega_{\delta_{1}}$ such that its image under the harmonic maps $h \circ f^{-1} \circ \log$ has finite length. That is, there exists a path $f^{-1}(\log \gamma(t))$ in $\Omega_{\delta}$ such that its image under $h$ has finite length.

Theorem 1.2. Let $h$ be a harmonic diffeomorphism from $\mathbb{C}$ into $\mathbb{H}^{2}$ with Hopf differential of the form

$$
\Phi=P_{1} \exp \left[P_{2} \exp \left[\cdots \exp \left[P_{k} \exp (Q)\right] \cdots\right]\right] d z^{2},
$$

where $Q(z)=a_{n} z^{n}+\cdots$ and $P_{j}, j=1, \ldots, k$ are polynomials and $k \geq 1$. Then $h$ is not surjective.

Proof. By making a change of parameter of the form $z \rightarrow r_{0} \exp \left(\sqrt{-1} \theta_{0}\right) z$ for some constants $r_{0}$ and $\theta_{0}$, we may assume that $Q=z^{n}+\sum_{j=1}^{n} a_{j} z^{n-j}$. As $P_{j}, j=1, \ldots, k$, are polynomials, there exists $R_{0}>0$ such that there 
is no zeros of any $P_{j}$ in the set $\left\{|z|>R_{0}\right\}$. Then for any $\delta>0$, one can define $g_{j}=\log P_{j}$ on the set $\Omega_{\delta}=\left\{|z|>R_{0},|\arg z|<\pi-\delta\right\}$ and the Hopf differential $\Phi$ can be written in the form required in Lemma 1.3. Therefore, there exists a path diverging to infinity in $\Omega_{\delta}$ such that its image under $h$ has finite length. Hence, $h$ is not surjective.

Let us finish the dicussion of this section by giving a different type of condition for the nonsurjectivity. Recall that a complex number $a$ in the extended complex plane is said to be an asymptotic value of an entire function $f(z)$ if there is a path $z(t), 0 \leq t<1$ such that $\lim _{t \rightarrow 1} z(t)=\infty$ and $\lim _{t \rightarrow 1} f(z(t))=a$. If $a$ is a finite number, then it is called a finite asymptotic value.

Theorem 1.3. Let $f$ be an entire function. Suppose there exist $\delta>0$ and $R>0$ such that

(i) $f$ has no finite asymptotic value in the domain

$$
\mathcal{R}=\left\{\zeta \mid \frac{\pi}{2}-\delta<\arg \zeta<\frac{\pi}{2}+\delta, \text { and }|\zeta|>R\right\} ; \quad \text { and }
$$

(ii) $f^{\prime}(z) \neq 0$ for all $z$ in $f^{-1}(\mathcal{R})$.

Suppose $h$ is an orientation harmonic diffeomorphism from $\mathbb{C}$ into $\mathbb{H}^{2}$ with Hopf differential $\left(f^{\prime}\right)^{2} d z^{2}$, then $h$ is not surjective.

Proof. Let $\Omega$ be a component of $f^{-1}(\mathcal{R})$. By (ii), $f$ is a local diffeomorphism on $\Omega$. By (i), we can conclude that every path in $\mathcal{R}$ begins at $\zeta_{0}$ can be lifted to a path in $\Omega$ which begins at a point $z_{0}$ with $f\left(z_{0}\right)=\zeta_{0}$. Since $\mathcal{R}$ is simply connected, $f$ maps $\Omega$ bijectively to $\mathcal{R}$. Hence $h \circ f^{-1}(\zeta)$ is a harmonic diffeomorphism from $\mathcal{R}$ into $\mathbb{H}^{2}$ with Hopf differential $d \zeta^{2}$. Moreover, $f^{-1}(\zeta) \rightarrow \infty$ if $\zeta \in \mathcal{R}$ and $\zeta \rightarrow \infty$. The result follows from Lemma 1.1.

\section{Maximal $\Phi$-radius and quasi-conformal harmonic maps.}

Let us recall the definition of maximal $\Phi$-radius of a holomorphic quadratic differential $\Phi$ on a domain in $\mathbb{C}$. Let $\Omega$ be a domain in $\mathbb{C}$ and let $\Phi=\phi d z^{2}$ be a holomorphic quadratic differential on $\Omega$. Let $z_{0} \in \Omega$ such that $\phi\left(z_{0}\right) \neq 0$. Choose a branch of $\sqrt{\phi}$ near $z_{0}$, and let

$$
w=f(z)=\int_{z_{0}}^{z} \sqrt{\phi(\zeta)} d \zeta
$$


Let $B(R)=\{w|| w \mid<R\}$ be the maximal disk in the $w$-plane such that $f^{-1}$ is a conformal diffeomorphism from $B(R)$ into $\Omega$. Then $R_{z_{0}, \Omega}=R$ is called the maximal $\Phi$-radius of $\Phi$ at $z_{0}$ with respect to $\Omega$ and $V_{z_{0}, \Omega}=f^{-1}(B(R))$ is called the maximal $\Phi$ disk around $z_{0}$ with respect to $\Omega$. We will drop the subscript $\Omega$ if this will not cause any confusion. Moreover, by convention if $\phi\left(z_{0}\right)=0$ we define $R_{z_{0}}=0$. In [Hn], it was proved that if $h$ is an orientation preserving harmonic diffeomorphism from a domain $\Omega$ in $\mathbb{C}$ into $\mathbb{H}^{2}$ with Hopf differential $\Phi$, and if $z_{n}$ is a sequence in $\Omega$ with $R_{z_{n}} \rightarrow \infty$, then the modulus the complex dilatation of $h$ at $z_{n}$ will tend to 1 . Conversely, one would like to know whether $h$ would be quasi-conformal on a set with bounded maximal $\Phi$-radius. In this section, we will prove that this is the case under certain assumptions. The result will be useful to study images of harmonic diffeomorphisms from $\mathbb{C}$ into $\mathbb{H}^{2}$.

Let $\Omega$ be a hyperbolic domain in $\mathbb{C}$, i.e. its universal cover is conformal to the unit disk. Let $\rho^{2}|d z|^{2}$ be the hyperbolic metric on $\Omega$, i.e. the complete metric with constant Gaussian curvature -1 . Then it is known that [Ah] for any $z \in \Omega$

$$
\rho(z) \leq \frac{2}{d(z, \partial \Omega)}
$$

where $d(z, \partial \Omega)$ is the Euclidean distance from $z$ to $\partial \Omega$. If in addition, we have

$$
\rho(z) \geq \frac{C}{d(z, \partial \Omega)}
$$

for some positive constant $C$ for all $z \in \Omega$, then we say that $\Omega$ is strongly hyperbolic. Please note that our definition is slightly different from that in $[A-M-M]$. It is shown in Theorem 5 of $[A-M-M]$ that if $\Omega$ is bounded hyperbolic and the diameters of the boundary components are uniformly bounded from below by a positive constant then $\Omega$ is strongly hyperbolic. Moreover, being strongly hyperbolic is conformally invariant:

Lemma 2.1. Let $\Omega_{1}$ and $\Omega_{2}$ be conformally equivalent domains. Suppose $\Omega_{1}$ is strongly hyperbolic, so is $\Omega_{2}$.

Proof. Obviously $\Omega_{2}$ is hyperbolic. Let $w=f(z)$ be a conformal diffeomorphism from $\Omega_{1}$ onto $\Omega_{2}$. Let $\rho_{2}^{2}|d w|^{2}$ be the hyperbolic metric on $\Omega_{2}$ and let $\rho_{1}^{2}|d z|^{2}$ be the hyperbolic metric on $\Omega_{1}$. Let $d_{1}(z)=\operatorname{dist}\left(z, \partial \Omega_{1}\right)$ and $d_{2}(w)=\operatorname{dist}\left(w, \partial \Omega_{2}\right)$, where both distances are Euclidean distances. Then by well-known fact [V, p. 147], we have

$$
\frac{1}{4} d_{1}(z)\left|f^{\prime}(z)\right| \leq d_{2}(f(z)) \text {. }
$$


Hence

$$
\begin{aligned}
\rho_{2}(f(z)) & =\left|f^{\prime}(z)\right|^{-1} \rho_{1}(z) \\
& \geq \frac{C}{d_{1}(z)\left|f^{\prime}(z)\right|} \\
& \geq \frac{C}{4} d_{2}(f(z))
\end{aligned}
$$

for some positive contant $C$, where we have used the fact that $\Omega_{1}$ is strongly hyperbolic. Hence $\Omega_{2}$ is strongly hyperbolic.

Let $\Omega$ be a strongly hyperbolic domain and let $\Phi=\phi d z^{2}$ be a holomorphic quadratic differential on $\Omega$ with hyperbolic metric $\rho^{2}|d z|^{2}$. For $z \in \Omega$, let $\|\Phi(z)\|=\rho^{-2}(z)|\phi|(z)$ be the norm of $\Phi$ at $z$ and let $\||\Phi|\|=$ $\sup _{z \in \Omega}\|\Phi\|(z)$. The following is proved in [A-M-M] (the equation (2) and the lemma 1.2)

Theorem (Anić-Marković-Mateljević). With the above notations and with $\Omega$ being the unit disk $\mathbb{D}$, there exists an absolute constant $C>0$ such that for any holomorphic quadratic differential $\Phi$ on $\mathbb{D}$ we have

$$
\|\Phi\|(z) \geq C^{-1} R_{z}^{2}
$$

for all $z \in \mathbb{D}$, and

$$
\||\Phi|\| \leq C R_{\infty}^{2}
$$

where $R_{\infty}=\sup _{z \in \mathbb{D}} R_{z}$.

They actually proved that (2.1) is true for any hyperbolic domain in $\mathbb{C}$. We will obtain a pointwise estimate for strongly hyperbolic domain which implies (2.2). The estimate will be useful in applications.

Proposition 2.1. Let $\Omega$ be a hyperbolic domain and let $\Phi=\phi d z^{2}$ be a holomorphic quadratic differential defined on $\Omega$. Then there exists an absolute positive constant $C$ such that for $z \in \Omega$

$$
R_{z} \leq C\|\Phi\|^{\frac{1}{2}}(z) .
$$

If in addition $\Omega$ is strongly hyperbolic then there is a positive constant $C^{\prime}$ depending only on $\Omega$ such that for $z \in \Omega$ with $\Phi(z) \neq 0$

$$
R_{z} \geq C^{\prime} \frac{\|\Phi\|^{2}(z)}{\|\Phi\|^{\frac{3}{2}}}
$$


We need the following lemmas.

Lemma 2.2. Denote $B(r)$ to be the set of complex numbers with modulus less than $r$. Let $f: B(r) \rightarrow \mathbb{C}$ be an analytic function, such that $f(0)=0$ and $f^{\prime}(0) \neq 0$. Suppose $|f(z)| \leq M$ for all $z$, then

(i) $f$ is one-to-one on $B\left(r_{1}\right)$, where $r_{1}=\frac{r^{2}\left|f^{\prime}(0)\right|}{8 M}$;

(ii) $f\left(B\left(r_{1}\right)\right) \supset B\left(\frac{r^{2}\left|f^{\prime}(0)\right|^{2}}{32 M}\right)$.

Proof. Let us first assume that $r=1$, and $f^{\prime}(0)=1$. Then

$$
f(z)=z+\sum_{n=2}^{\infty} a_{n} z^{n} .
$$

By Cauchy theorem, we have $\left|a_{n}\right| \leq M$, and $1 \leq M$. Suppose $z_{1} \neq z_{2}$ are in $B\left(\frac{1}{8 M}\right)$, and $r=\max \left\{\left|z_{1}\right|,\left|z_{2}\right|\right\}$, then

$$
\begin{aligned}
\left|f\left(z_{1}\right)-f\left(z_{2}\right)\right| & =\left|\left(z_{1}-z_{2}\right)+\sum_{n=2}^{\infty} a_{n}\left(z_{1}^{n}-z_{2}^{n}\right)\right| \\
& \geq\left|z_{1}-z_{2}\right|\left|1-M \sum_{n=2}^{\infty} n r^{n-1}\right| \\
& =\left|z_{1}-z_{2}\right|\left|1-M r \frac{2-r}{(1-r)^{2}}\right| \\
& \geq\left|z_{1}-z_{2}\right|(1-8 M r) \\
& >0
\end{aligned}
$$

where we have used the facts that $M \geq 1$, and $r<\frac{1}{8 M}<\frac{1}{2}$. Hence $f$ is one-to-one on $B\left(\frac{1}{8 M}\right)$. Using the fact that the Koebe's constant is $\frac{1}{4}[\mathrm{~V}$, p. 149], we have $f\left(B\left(\frac{1}{8 M}\right)\right)$ contains $B\left(\frac{1}{32 M}\right)$. In general, if $f$ is defined on $B(r)$ with $f(0)=0$ and with $b=f^{\prime}(0) \neq 0$. Define $\tilde{f}(\zeta)=\frac{f(r \zeta)}{r b}$ for $\zeta \in \mathbb{D}$. Then $\tilde{f}(0)=0$, and $\tilde{f}^{\prime}(0)=1$. Let $M_{1}=\frac{M}{r|b|}$, then $M_{1} \geq|\tilde{f}(\zeta)|$ for all $\zeta$. Hence $\tilde{f}$ is one-to-one on $B\left(\frac{1}{8 M_{1}}\right)$ and $\tilde{f}\left(B\left(\frac{1}{8 M_{1}}\right)\right)$ contains $B\left(\frac{1}{32 M_{1}}\right)$. Therefore $f$ is one-to-one on $B\left(\frac{r}{8 M_{1}}\right)=B\left(\frac{r^{2}\left|f^{\prime}(0)\right|}{8 M}\right)=B\left(r_{1}\right)$, and $f\left(B\left(r_{1}\right)\right)$ contains $B\left(\frac{r|b|}{32 M_{1}}\right)=B\left(\frac{r^{2}\left|f^{\prime}(0)\right|^{2}}{32 M}\right)$.

The following lemma is proved in [A-M-M, see Lemma 1.2]. 
Lemma 2.3. Let $f: B(r) \rightarrow \mathbb{C}$ be analytic, and $M \geq|f(z)|$ for all $z$. Suppose $f(0) \neq 0$, then $f(z) \neq 0$ for all $z \in B\left(\frac{r|f(0)|}{2 M}\right)$.

Proof of Proposition 2.1. (2.3) was proved in the Lemma 2.3 of [A-M-M]. In order to prove (2.4), let $z_{0} \in \Omega$, with $\phi\left(z_{0}\right) \neq 0 . \phi$ is analytic on $B\left(z_{0}, r_{0}\right)$ where $r_{0}=\frac{1}{2} d\left(z_{0}, \partial \Omega\right)$, where $d$ is the Euclidean distance. By Lemma 2.3, $\phi$ is never zero on $B\left(z_{0}, r\right)$, where

$$
r=\frac{r_{0}\left|\phi\left(z_{0}\right)\right|}{2 M_{0}}
$$

and $M_{0}=\sup _{B\left(z_{0}, r_{0}\right)}|\phi|$. Hence we can take a branch of square root of $\phi$ in $B\left(z_{0}, r\right)$. Let $f(z)=\int_{z_{0}}^{z} \sqrt{\phi}(\zeta) d \zeta$, for $z \in B\left(z_{0}, r\right)$, then $f$ is analytic, $f\left(z_{0}\right)=0$ and $\left|f^{\prime}\left(z_{0}\right)\right|=\left|\phi\left(z_{0}\right)\right|^{\frac{1}{2}} \neq 0$. By Lemma $2.2, f$ is one-to-one on $B\left(r_{1}\right)$ where $r_{1}=\frac{r^{2}\left|f^{\prime}\left(z_{0}\right)\right|}{8 M_{1}}$, where $M_{1}=\sup _{B\left(z_{0}, r\right)}|f|$. Moreover, $f\left(B\left(r_{1}\right)\right)$ contains the disk $B(R)=B\left(\frac{r^{2}\left|f^{\prime}\left(z_{0}\right)\right|^{2}}{M_{1}}\right)$. Now $M_{1} \leq r M_{0}^{\frac{1}{2}}$ implies that

$$
R_{z_{0}} \geq R=\frac{r^{2}\left|\phi\left(z_{0}\right)\right|}{32 r M_{0}^{\frac{1}{2}}}=\frac{r_{0}\left|\phi\left(z_{0}\right)\right|^{2}}{64 M_{0}^{\frac{3}{2}}} .
$$

This will imply the proposition because $\Omega$ is strongly hyperbolic.

From the proof of the proposition, we have the following corollary which will be used in $\S 3$ and $\S 4$.

Corollary 2.1. Suppose $\phi$ is analytic on $B_{z_{0}}(R)$ such that $\alpha|\phi|\left(z_{0}\right) \geq|\phi|(z)$ for some constant $\alpha>0$ for all $z \in B_{z_{0}}(R)$. Let $\Phi=\phi d z^{2}$. Then the maximal $\Phi$-radius of $z_{0}$ with respect to $B_{z_{0}}(R)$ is bounded below by $\frac{\left.R|\phi|\right|^{\frac{1}{2}}\left(z_{0}\right)}{64 \alpha^{\frac{3}{2}}}$.

Proof. This is a direct consequence of (2.5).

Lemma 2.4. Let $\Omega$ be a simply connected domain in $\mathbb{C}$ and $\Phi=\phi d z^{2}$ be a holomorphic quadratic differential on $\Omega$. Let $z_{0} \in \Omega$ such that $\phi\left(z_{0}\right) \neq 0$ and let $R$ be the maximal $\Phi$-radius of $z_{0}$ with maximal $\Phi$-disk $V$. Suppose $R<\infty$ and suppose

$$
w=\psi(z)=\int_{z_{0}}^{z} \sqrt{\phi} d \zeta
$$


$z \in V$. Then for any $0<\delta<R$, there exists a point $z \in V$ with $|\psi(z)|=\delta$ such that the $\Phi$-radius of $z$ is exactly $R-\delta$.

Proof. By the definitions of $V$ and $R, \psi^{-1}: \mathbb{D}_{R} \rightarrow V$ is a bijective conformal diffeomorphism, where

$$
\mathbb{D}_{R}=\{w|| w \mid<R\}
$$

It is easy to see that if $z \in V$ with $|\psi(z)|=\delta$ then the $\Phi$-radius of $z$ is at least $R-\delta$. Suppose the lemma is not true, then $R_{z}>R-\delta$ for any $z \in \psi^{-1}(\{w|| w \mid=\delta\})$. Because $R_{z}$ is continuous, there is $\epsilon>0$ such that $R_{z} \geq R-\delta+\epsilon$ for all $z$ with $|\psi(z)|=\delta$. Hence $\psi^{-1}$ can be extended to an analytic function from $\mathbb{D}_{R+\epsilon}$ to $\Omega$ such that it is a local diffeomorphism. In particular, $\phi$ is not zero in $\psi^{-1}\left(\mathbb{D}_{R+\epsilon}\right)$. By the definition of $R$, there exist two sequences $w_{n}$ and $\tilde{w}_{n}$ such that for each $n$ both $w_{n}$ and $\tilde{w}_{n}$ are in $\mathbb{D}_{R+\epsilon / n}, w_{n} \neq \tilde{w}_{n}$ but $\psi^{-1}\left(w_{n}\right)=\psi^{-1}\left(\tilde{w}_{n}\right)$. Without loss of generality, we may assume that $w_{n} \rightarrow a$ and $\tilde{w}_{n} \rightarrow b$, and

$$
\lim _{n \rightarrow \infty} \psi^{-1}\left(w_{n}\right)=\lim _{n \rightarrow \infty} \psi^{-1}\left(\tilde{w}_{n}\right)=c .
$$

Since $\psi^{-1}$ is a local diffeomorphism, $a \neq b$. Note that $a$ and $b$ are in $\overline{\mathbb{D}}_{R}$. Let $\gamma$ be the straight line joining $a$ and $b$ and let $\Gamma=\psi^{-1}(\gamma)$. Then $\Gamma$ is a smooth simple closed curve in $\Omega$ because $\psi^{-1}$ is one-to-one on $\mathbb{D}_{R}$. Let $\theta$ be the interior angle at $c$. Apply the Gauss-Bonnet Theorem for the metric $(|\phi|+\eta)|d z|^{2}$ on $\Omega$ with $\eta>0$, we have

$$
-\frac{1}{2} \int_{\Omega_{1}} \Delta \log (|\phi|+\eta)+\int_{\Gamma} \kappa_{\eta}=\pi+\theta
$$

where $\Omega_{1}$ is the interior of $\Gamma, \kappa_{\eta}$ is the geodesic curvature of $\Gamma$ with respect to the metric $(|\phi|+\eta)|d z|^{2}$ and $\Delta$ is the Euclidean Laplacian. Here we have used the fact that $\Omega$ is simply connected. Let $c_{1}, \ldots, c_{\ell}$ be the zeros of $\phi$ inside $\Omega_{1}$ with multiplicities $k_{1}, \ldots, k_{\ell}$ with $k_{j} \geq 0$. Let $r>0$ be small enough so that $1 \leq j \leq \ell$ the disks $D_{j}$ of radius $r$ and centers at $c_{j}$ are 
disjoint and are inside of $\Omega_{1}$. Then we have

$$
\begin{aligned}
\pi+\theta & =-\frac{1}{2} \sum_{j=1}^{\ell} \int_{D_{j}} \Delta \log (|\phi|+\eta)-\frac{1}{2} \int_{\Omega_{1} \backslash \cup_{j=1}^{\ell} D_{j}} \Delta \log (|\phi|+\eta)+\int_{\Gamma} \kappa_{\eta} \\
& =-\frac{1}{2} \sum_{j=1}^{\ell} \int_{\partial D_{j}} \frac{\partial}{\partial r} \log (|\phi|+\eta)-\frac{1}{2} \int_{\Omega_{1} \backslash \cup_{j=1}^{\ell} D_{j}} \Delta \log (|\phi|+\eta)+\int_{\Gamma} \kappa_{\eta} \\
& \rightarrow-\frac{1}{2} \sum_{j=1}^{\ell} \int_{\partial D_{j}} \frac{\partial}{\partial r} \log |\phi|+\int_{\Gamma} \kappa
\end{aligned}
$$

as $\eta \rightarrow 0$, where $\kappa$ is the geodesic curvature with respect to the metric $|\phi||d z|^{2}$ and we have used the fact that $\log |\phi|$ is harmonic. Since $|\phi||d z|^{2}$ is the pull-back metric under $\psi^{-1}$ of the flat metric in the $\mathbb{D}_{R}$, we have $\kappa=0$ on $\Gamma$. Let $r \rightarrow 0$, we conclude that

$$
\pi+\theta=-\sum_{j=1}^{\ell} k_{j} \pi .
$$

Since $\theta \geq 0$ and $k_{j} \geq 0$ for all $j$, this is impossible.

Theorem 2.1. Let $\Omega$ be a strongly hyperbolic domain in $\mathbb{C}$ with hyperbolic metric $e^{2 v}|d z|^{2}$ and $\Omega_{1} \subset \Omega$. Let $h$ be an orientation preserving harmonic diffeomorphism from $\mathbb{C}$ into $\mathbb{H}^{2}$ and let $w=\log |\partial h|$, where $|\partial h|$ is the norm of $\partial h$ with respect to the Euclidean metric on $\Omega$ and hyperbolic metric on $\mathbb{H}^{2}$. Let $\Phi=\phi d z^{2}$ be the Hopf differential of $h$ and let $R_{z}$ be the maximal $\Phi$-radius of $z$ with respect to $\mathbb{C}$. Suppose $\sup _{z \in \Omega} R_{z}=R<\infty$ and $w \geq v-C$ on $\Omega_{1}$ for some constant $C$ and $\inf _{z \in \mathbb{C} \backslash \Omega_{1}} R_{z}>0$. Then $h$ is quasi-conformal on $\Omega_{1}$.

Proof. Suppose that $h$ is not quasi-conformal on $\Omega_{1}$. Then there exists $z_{n} \in \Omega_{1}$ such that $\phi\left(z_{n}\right) e^{-2 w\left(z_{n}\right)} \rightarrow 1$ as $n \rightarrow \infty$. Since $R_{z_{n}} \leq R$, we may assume that $\lim _{n \rightarrow \infty} R_{z_{n}}=R_{0}$. Suppose $R_{0}>0$. Let $V_{z_{n}}$ be the maximal $\Phi$-disk with image $\mathbb{D}_{R_{z_{n}}}$ and let $\zeta=\int_{z_{n}}^{z} \sqrt{\phi} d z=\psi_{n}(z)$. Let $\tilde{w}_{n}(\zeta)=\left(w-\frac{1}{2} \log |\phi|\right)\left(\psi_{n}^{-1}(\zeta)\right)>0$ which is considered as a function on $\mathbb{D}_{R_{z_{n}}}$. Then $\tilde{w}_{n}>0$ and

$$
\Delta_{\zeta} \tilde{w}_{n}=e^{2 \tilde{w}_{n}}-e^{-2 \tilde{w}_{n}}
$$


Then $\tilde{w}_{n}$ are locally uniformly bounded by proposition 1.5 in [T-W1]. Passing to a subsequence if necessary, $\tilde{w}_{n}$ converges uniformly on compact subsets of $\mathbb{D}_{R_{0}}$. Since $\tilde{w}_{n}(0) \rightarrow 0$, by mean value inequality, $\tilde{w}_{n} \rightarrow 0$ uniformly on compact sets of $\mathbb{D}_{R_{0}}$. By Lemma 2.4, for each $n$ there exists $\zeta_{n}$ with $\left|\zeta_{n}\right|=\frac{1}{2} R_{z_{n}}$ such that the $\Phi$-radius of $z_{n}^{\prime}=\psi_{n}^{-1}\left(\zeta_{n}\right)$ is $\frac{1}{2} R_{z_{n}}$. Moreover, we still have $\phi\left(z_{n}^{\prime}\right) e^{-2 w\left(z_{n}^{\prime}\right)} \rightarrow 1$. Continue in this way and by a diagonal process, if $h$ is not quasi-conformal on $\Omega_{1}$, then we can find $z_{n} \in \mathbb{C}$ such that

$$
\lim _{n \rightarrow \infty}\left|\phi\left(z_{n}\right)\right| e^{-2 w\left(z_{n}\right)}=1
$$

and

$$
\lim _{n \rightarrow \infty} R_{z_{n}}=0 \text {. }
$$

Since $\inf _{z \in \mathbb{C} \backslash \Omega_{1}} R_{z}>0$, we may assume that $z_{n} \in \Omega_{1}$ for all $n$. By Proposition 2.1 , we have

$$
\begin{aligned}
R_{z_{n}} & \geq C_{3} \frac{\|\Phi\|^{2}\left(z_{n}\right)}{\|\left.|\Phi|\right|^{\frac{3}{2}}} \\
& \geq \frac{C_{4}}{R^{3}}\left[\left|\phi\left(z_{n}\right)\right| e^{-2 v\left(z_{n}\right)}\right]^{2} \\
& \geq \frac{C_{5}}{R^{3}}\left[\left|\phi\left(z_{n}\right)\right| e^{-2 w\left(z_{n}\right)}\right]^{2},
\end{aligned}
$$

where the norm of $\Phi$ is taken with respect to the metric $e^{2 v}|d z|^{2}$ on $\Omega$. Here we have used (2.3), (2.4), the fact that the $\Phi$-radius with respect to $\Omega_{1}$ or $\Omega$ is no greater than the $\Phi$-radius with respect to $\mathbb{C}$, the assumption that $R_{z}$ are uniformly bounded by $R$ on $\Omega$ and that $w \geq v-C$ on $\Omega_{1}$. Let $n \rightarrow \infty$ in (2.8), we have a contradiction because of (2.6) and (2.7). This completes the proof of the theorem.

Remark 2.1. In the theorem, we may replace $\mathbb{C}$ by the unit disk. Moreover, suppose $\Omega$ is a subset of $\mathbb{C}$ (respectively $\mathbb{H}^{2}$ ) and $h$ is an orientation preserving harmonic diffeomorphism from $\mathbb{C}$ (respectively $\mathbb{H}^{2}$ ) into $\mathbb{H}^{2}$ such that $|\partial h|^{2}|d z|^{2}$ is complete in $\mathbb{C}$ (respectively $\mathbb{H}^{2}$ ), where the norm is taken with respect to the Euclidean metric in the domain. Then the assumption that $w \geq v-C$ on $\Omega_{1}$ in the theorem can be replaced by $w \geq v-C$ on $\partial \Omega_{1}$ by the comparison principle in [Wn].

By (2.3), which was proved in [A-M-M], and the above remark, we obtain a new proof of the following result in [Wn] as a corollary of Theorem 2.1. 
Corollary 2.2. Let $h$ be an orientation preserving harmonic diffeomorphism on $\mathbb{H}^{2}$ with Hopf differential $\Phi$ such that $|\partial h|^{2}|d z|^{2}$ is complete. Suppose $|\|\Phi \mid\|<\infty$, then $h$ is quasi-conformal. Here the norm of $\partial h$ is taken with respect to the Euclidean metric in the domain and the norm of $\Phi$ is taken with respect to the Poincaré metric while $\||\Phi|||$ is taken with respect to the Poincaré metric.

\section{Image of a harmonic diffeomorphism with Hopf differential $P \exp (Q) d z^{2}$.}

Let $h$ be an orientation preserving harmonic diffeomorphism from $\mathbb{C}$ into $\mathbb{H}^{2}$ with Hopf differential $\Phi=\phi d z^{2}=P \exp (Q) d z^{2}$ where $P$ and $Q$ are polynomials. By the result of $\S 1$, we know that $h$ is not surjective. Assume that $|\partial h|^{2}|d z|^{2}$ is complete on $\mathbb{C}$. In [HTTW], it was proved that if $Q$ is a constant, that is, if $\phi$ is a polynomial of degree $m$, then the closure of the image of $h$ in $\overline{\mathbb{H}^{2}}$ is the convex hull of an ideal polygon with $m+2$ vertices in $\mathbb{H}^{2}$. The result is generalized from $\mathbb{C}$ to surfaces with finite total curvature and in higher dimensions in [L-W1, L-W2]. The assumption that $\phi$ is a polynomial is equivalent to the fact that $h$ is of polynomial growth. Let $A$ be the intersection of the closure of the image of $h$ with the geometric boundary of $\partial \mathbb{H}^{2}$. If $Q$ is not constant, then $A$ will no longer be a finite set. In this section, we will prove that in this case, $A$ is a countable set with exactly $n$ distinct accumulation points where $n$ is the degree of $Q$. In fact, we will prove that the result is true for a larger class of harmonic diffeomorphisms.

First we need a lemma. For $\alpha \geq 0$, let

$$
\mathbb{C}_{\alpha}=\{z \mid \Re z>\alpha,-\infty<\Im z<\infty\} .
$$

Let $h$ be an orientation preserving harmonic diffeomorphism from $\mathbb{C}_{\alpha}$ into $\mathbb{H}^{2}$ with Hopf differential $\Phi=\exp (Q) d z^{2}$ with $Q(z)=z+q(z)$ such that $|q(z)| \leq g(\Re z)$ for some nonnegative function $g$ with $\lim _{t \rightarrow \infty} g(t)=0$.

Lemma 3.1. With the above notations and assumptions, we have the following:

(i) There exist distinct points $p_{k} \in \partial \mathbb{H}^{2}$ with $k=0, \pm 1, \pm 2, \ldots$ such that for any $\pi>\delta>0$ and for any sequence $z_{n} \in \mathbb{C}_{\alpha}$ with $(2 k-1) \pi+\delta<$ $\Im z_{n}<(2 k+1) \pi-\delta$ and $z_{n} \rightarrow \infty$, then $h\left(z_{n}\right) \rightarrow p_{k}$ as $n \rightarrow \infty$. Moreover, the $p_{k}$ 's are monotone in $\mathbb{S}^{1}$. 
(ii) For all $\delta>0$ and $\epsilon>0$, there is a $>0$ such that for any integer $k$, if $2 k \pi+\delta<\Im z<2(k+1) \pi-\delta$, then $d_{\mathbb{H}^{2}}\left(h(z), \gamma_{k}\right) \leq \epsilon$ for all $z \in \mathbb{C}_{\alpha}$ with $\Re z>a$, where $\gamma_{k}$ is the geodesic joining $p_{k}$ and $p_{k+1}$.

(iii) There is $b>0$ such that if $z_{n} \in \mathbb{C}_{\alpha}, \Re z_{n} \geq b$ and $\Im z_{n} \rightarrow+\infty$ (respectively $\left.\Im z_{n} \rightarrow-\infty\right)$, then $\lim _{n \rightarrow \infty} h\left(z_{n}\right)=p_{+}$(respectively $\left.\lim _{n \rightarrow \infty} h\left(z_{n}\right)=p_{-}\right)$, where $p_{+}=\lim _{k \rightarrow \infty} p_{k}$ and $p_{-}=\lim _{k \rightarrow-\infty} p_{k}$.

Proof. For simplicity, we assume $\alpha=0$. To prove the existence of those $p_{k} \in \partial \mathbb{H}^{2}$ in (i), we apply Lemma 1.2 with $\theta=0$ and $A=2 \pi$ to conclude that for any $\pi>\delta>0$, there exists $x_{0}>0$ such that if $z_{0}=x_{0}$ and $\zeta(z)=\int_{z_{0}}^{z} \exp \left(\frac{1}{2} Q(\xi)\right) d \xi+\exp \left(\frac{1}{2} x_{0}\right)$, then $\zeta$ is injective on $\mathcal{S}_{\frac{1}{4} \delta}$, and $\zeta\left(\mathcal{S}_{\frac{1}{4} \delta}\right) \supset$ $\mathcal{R}_{\frac{1}{2} \delta} \supset \zeta\left(\mathcal{S}_{\delta}\right)$ where $\mathcal{S}_{\delta}$ and $\mathcal{R}_{\delta}$ etc. are defined as in Lemma 1.2 (with $A=2 \pi$ and $\theta=0)$. Then $h(z(\zeta))$ is an orientation preserving harmonic diffeomorphism from $\zeta\left(\mathcal{S}_{\frac{1}{4} \delta}\right)$ into $\mathbb{H}^{2}$ with Hopf differential $\Phi=d \zeta^{2}$. Note that the maximal $\Phi$-radius of any point $\zeta=u+\sqrt{-1} v$ in $\mathcal{R}_{\frac{1}{2} \delta}$ is at least $u-C_{1}$ for some constant $C_{1}$ depending only on $\delta$. As in [HTTW, p. 109], we can prove that the image under $h$ of any horizontal half line $\zeta(t)=t+\sqrt{-1} v_{0}$ in $\mathcal{R}_{\frac{1}{2} \delta}$ with $t$ being larger than some constant is asymptotically a geodesic near infinity and tends to a point in $\partial \mathbb{H}^{2}$ as $t \rightarrow \infty$. By the proof of Lemma 1.1 , we can conclude that the image of any vertical line $u=$ constant in $\mathcal{R}_{\frac{1}{2} \delta}$ under $h$ has uniformly bounded length. Hence if $\zeta_{n} \in \mathcal{R}_{\frac{1}{2} \delta}, \zeta_{n} \rightarrow \infty$ then $h\left(z\left(\zeta_{n}\right)\right) \rightarrow p_{0}$ for some $p_{0} \in \partial \mathbb{H}^{2}$. Since $\zeta\left(\mathcal{S}_{\delta}\right) \subset \mathcal{R}_{\frac{1}{2} \delta}$ and $z_{n} \rightarrow \infty$ implies that $\zeta\left(z_{n}\right) \rightarrow \infty$ for $z_{n} \in \mathcal{S}_{\delta}$, we have

$$
\lim _{n \rightarrow \infty} h\left(z_{n}\right)=p_{0}
$$

Similarly, one can prove that for any integer $k$ there exists $p_{k} \in \partial \mathbb{H}^{2}$ such that for any $\delta>0$ and $z_{n}$ with $(2 k-1) \pi+\delta<\Im z_{n}<(2 k+1) \pi-\delta$ such that $z_{n} \rightarrow \infty$, then

$$
\lim _{n \rightarrow \infty} h\left(z_{n}\right)=p_{k}
$$

To prove the remaining of (i) and (ii), we use Lemma 1.2 again to conclude that for all $\delta>0$ small enough, there exist $a_{j}>0, b_{j}>0, j=1,2$ such that for any integer $k$, there is a analytic function $\zeta=\zeta^{(k)}(z)$ which maps

$$
\mathcal{S}_{1}=\left\{z \mid \Re z>a_{1},(2 k-1) \pi+\frac{1}{2} \delta<\Im z<(2 k+3) \pi-\frac{1}{2} \delta\right\}
$$


and

$$
\mathcal{S}_{2}=\left\{z \mid \Re z>a_{2}, 2 k \pi-\delta<\Im z<2(k+1) \pi+\delta\right\}
$$

injectively into $\zeta$-plane. Moreover, $\zeta\left(\mathcal{S}_{1}\right) \supset \mathcal{R}_{1}, \zeta\left(\mathcal{S}_{2}\right) \subset \mathcal{R}_{2}$, for $j=1,2$. Here

$$
\begin{gathered}
\mathcal{R}_{1}=\left\{\zeta|| \zeta \mid>b_{1}, \frac{1}{2}(2 k-1) \pi+\delta<\arg \zeta<\frac{1}{2}(2 k+3) \pi-\delta\right\}, \\
\mathcal{R}_{2}=\left\{\zeta|| \zeta \mid>b_{2}, \frac{1}{2}\left(2 k \pi-\frac{3}{2} \delta\right)<\arg \zeta<\frac{1}{2}\left(2(k+1) \pi+\frac{3}{2} \delta\right)\right\} .
\end{gathered}
$$

Moreover, the Hopf differential $\Phi$ of $h$ in the $\zeta$ coordinates is of the form $d \zeta^{2}$. We will write $h(\zeta)$ instead of $h(z(\zeta))$ if no confusion will arise. Let us consider the case when $k$ is even. The case that $k$ is odd is similar. By the previous result, we know that if $\zeta_{n} \in \mathcal{R}_{1}$ with $\Re \zeta_{n} \rightarrow \infty$ along a half line $\Im \zeta=$ constant, then $h\left(\zeta_{n}\right) \rightarrow p_{k}$, and if $\Re \zeta_{n} \rightarrow-\infty$, then $h\left(\zeta_{n}\right) \rightarrow p_{k+1}$.

In order to prove that $p_{k} \neq p_{k+1}$ and that $p_{k}$ is monotone, we notice that the length of the curve $h(z(\zeta))$ is infinite where $\zeta=u+\sqrt{-1} v_{1}$ with $v_{1}$ to be a constant and $-\infty<u<\infty$. Moreover, by [Wf, M] or [HTTW, p. 109], the geodesic curvature of this curve is bounded by $\epsilon$ provided $v_{1}$ is large. From this, it is easy to see that $p_{k} \neq p_{k+1}$. Since $h$ is an orientation preserving diffeomorphism, we conclude that $p_{k} \neq p_{j}$ if $k \neq j$, and $p_{k}$ is monotone on $\mathbb{S}^{1}$. In particular, $p_{+}=\lim _{k \rightarrow \infty} p_{k}$ and $p_{-}=\lim _{k \rightarrow-\infty} p_{k}$ exist.

To prove (ii), we observe that for any $C>0$ there is $v_{0}>0$ independent of $k$ such that the $\Phi$-radius of $\zeta \in \mathcal{R}_{1}$ is larger than $C$ for all $\zeta$ with $\Im \zeta>v_{0}$. By the argument in [HTTW, p. 102], we conclude that for any $\epsilon>0$, there is $v_{0}>0$ independent of $k$ such that if $\Im \zeta>v_{0}$, then $\left.d_{\mathbb{H}^{2}}(h(\zeta)), \gamma_{k}\right) \leq \epsilon$, where $\gamma_{k}$ is the geodesic joining $p_{k}$ and $p_{k+1}$. From the proof of Lemma 1.2, we see that given $v_{0}$, there exists $a>0$ independent of $k$ such that if $z \in \mathcal{S}_{2}$ and $\Re z>a$, then $\Im \zeta(z)>v_{0}$. From this we can conclude that (ii) is true.

In order to prove (iii), let $\delta>0$ as above but small and let $b=a_{2}$ which is in the definition of $\mathcal{S}_{2}$. Suppose $z_{n} \in \mathbb{C}_{\alpha}$ with $\Re z_{n}>b$. Let $k_{n}$ be such that $2 k_{n} \pi \leq \Im z_{n}<2\left(k_{n}+1\right) \pi$. Then $\lim _{n \rightarrow \infty} k_{n}=\infty$. For each $n$, let $\zeta=\zeta^{\left(k_{n}\right)}$ as above then $\zeta_{n}=\zeta\left(z_{n}\right)$ can be defined and $\zeta_{n} \in \mathcal{R}_{2}$. By Lemma 1.1, for all $\zeta \in \mathcal{R}_{2}$ with $\Im \zeta>0$ and $\Re \zeta=\Re \zeta_{n}, d_{\mathbb{H}^{2}}\left(h\left(\zeta_{n}\right), h(\zeta)\right) \leq C_{2}$ for some constant $C_{2}$ independent of $n$. From (ii), we conclude that $d_{\mathbb{H}^{2}}\left(h\left(\zeta_{n}\right), \gamma_{k_{n}}\right) \leq C_{3}$ for some constant $C_{3}$ independent of $n$. From this, the result follows.

Theorem 3.1. Let $h$ be an orientation preserving harmonic diffeomorphism from $\mathbb{C}$ into $\mathbb{H}^{2}$ with Hopf differential $\Phi=\phi d z^{2}=P \exp (Q) d z^{2}$ such that $|\partial h|^{2}|d z|^{2}$ is complete on $\mathbb{C}$ and such that 
(i) $Q(z)=z^{n}+\sum_{j=1}^{n-1} a_{j} z^{n-j}$ is a polynomial of degree $n \geq 1$;

(ii) $P \not \equiv 0$ is an entire function with order $\rho<n$; and

(iii) there exists $\frac{\pi}{2 n}>\delta>0$ and $R_{0}>0$ such that

$$
\Sigma \cap\left\{z|| z \mid>R_{0} \text { and }\left|\arg z-\frac{2 k \pi}{n}\right|<\frac{\pi}{2 n}+\delta\right\}=\emptyset
$$

for all $0 \leq k \leq n-1$, where $\Sigma$ is the set of all zeros of $P$.

Then the closure of the image of $h$ is the convex hull of a countable set $A$ of $\partial \mathbb{H}^{2}$ with exactly $n$ accumulation points.

Proof. We claim that for any $\epsilon>0$ with $n \epsilon<\frac{\pi}{2}$, there exists a constant $C_{1}>0$ such that the maximal $\Phi$-radius $R_{z}$ of $z$ satisfies

$$
R_{z} \leq C_{1}
$$

for all $z \in W_{k}, 0 \leq k \leq n-1$, where $W_{k}$ is the wedge

$$
W_{k}=\left\{z|| \arg z-\frac{(2 k+1) \pi}{n} \mid<\frac{\pi}{2 n}-\epsilon\right\} .
$$

for $0 \leq k \leq n-1$. To prove the claim, note that there exists $\tau>0$ such that for $z \in W_{k}, \Re\left(z^{n}\right) \leq-\tau|z|^{n}$. By the assumptions (i) and (ii), for any $z \in W_{k}$, let $\gamma$ be the half ray $\gamma(t)=t \exp (\sqrt{-1} \arg z)$ for $t \geq|z|$, then

$$
\begin{aligned}
& \int_{|z|}^{\infty}|\phi|^{\frac{1}{2}}(\gamma(t)) d t \\
& \leq \int_{|z|}^{\infty} \exp \left(-\frac{1}{2} \tau t^{n}+C_{2}\left(1+t^{n-1}+t^{\tilde{\rho}}\right)\right) d t, \text { for any } \rho<\tilde{\rho}<n, \\
& \leq C_{3}
\end{aligned}
$$

where $C_{2}$ and $C_{3}$ are constants independent of $z$. Hence the maximal $\Phi$ radius of $z \in W_{k}$ is uniformly bounded. This proves the claim (3.1).

Next, for each $0 \leq k \leq n-1$, and for $\delta>4 \epsilon>0$, let

$$
V_{k, \epsilon}=\left\{z|| \arg z-\frac{2 k \pi}{n} \mid<\frac{\pi}{2 n}+\epsilon\right\} .
$$

Define $V_{k, 4 \epsilon}$ similarly. By assumption (iii), we can take a branch of $\log P$ in $\left\{z \in V_{k, 4 \epsilon}|| z \mid>R_{0}\right\}$. As in the proof of Theorem 1.1, there exist 
positive constants $R_{2}>R_{1}>R_{0}, T_{2}>T_{1}, \epsilon_{1}>0$ and a conformal map $\zeta_{k}(z)$ which is of polynomial growth as a function of $z$ and which will map $\mathcal{S}_{1}^{(k)}=\left\{z \in V_{k, 4 \epsilon}|| z \mid>R_{1}\right\}$ injectively onto its image. For simplicity, we write $\zeta=\zeta_{k}$. Moreover, if

$$
\begin{gathered}
\mathcal{S}_{2}^{(k)}=\left\{z \in V_{k, \epsilon}|| z \mid>R_{2}\right\} \\
\mathcal{R}_{1}=\left\{\zeta|| \arg \zeta \mid<\frac{\pi}{2}+2 \epsilon_{1} \text { and }|\zeta|>T_{1}\right\}
\end{gathered}
$$

and

$$
\mathcal{R}_{2}=\left\{\zeta|| \arg \zeta \mid<\frac{\pi}{2}+\epsilon_{1} \text { and }|\zeta|>T_{2}\right\}
$$

then $\zeta\left(\mathcal{S}_{1}^{(k)}\right) \supset \mathcal{R}_{1} \supset \zeta\left(\mathcal{S}_{2}^{(k)}\right) \supset \mathcal{R}_{2}$. Also, in $\mathcal{R}_{1}$ the Hopf differential of $h$ is of the form $\Phi=\exp \left(\zeta+Q_{1}(\zeta)\right) d \zeta^{2}$ where $Q_{1}(\zeta) \rightarrow 0$ as $\zeta \rightarrow \infty$. Choose $a>b>T_{2}$. As in the proof of (3.1), we have

$$
R_{z} \leq C_{2}
$$

for some constant $C_{2}$ for all $z \in \mathcal{S}_{2}^{(k)} \cap \zeta^{-1}(\{\Re \zeta \leq a\})$. Moreover, on $\Re \zeta=b,\left|\exp \left(\zeta+Q_{1}(\zeta)\right)\right| \geq C_{3}$ for some positive constant $C_{3}$. Hence if $\tilde{w}=\log \left|\partial_{\zeta} h\right|$ and if $e^{2 \tilde{v}}|d \zeta|^{2}$ is the hyperbolic metric on $\zeta\left(\mathcal{S}^{(k)}\right) \cap\{\Re \zeta>a\}$, then $\tilde{w} \geq \tilde{v}-C_{4}$ for some constant $C_{4}$ because $e^{-2 \tilde{w}(\zeta)}\left|\exp \left(\zeta+Q_{1}(\zeta)\right)\right|<1$ and $\tilde{v} \leq C$ on $\Re z=b$ for some positive constant $C$. Let $\Gamma_{k}=\zeta^{-1}(\{\Re z=a\})$ and $\gamma_{k}=\zeta^{-1}(\{\Re \zeta=b\})$. Note that for fixed $c>T_{2}, \arg \left(\zeta^{-1}(c+\sqrt{-1} t)\right) \rightarrow$ $\left(2 k \pm \frac{1}{2}\right) \pi / n$ as $t \rightarrow \pm \infty$. Let $\Omega$ be the component containing the origin of $\mathbb{C} \backslash \cup_{k=0}^{n-1} \Gamma_{k}$, and let $\Omega_{1}$ be the component containing the origin of $\mathbb{C} \backslash \cup_{k=0}^{n-1} \gamma_{k}$. By (3.1) and (3.2) if we choose $\epsilon>0$ in (3.2) and then choose $\epsilon>0$ in (3.1) small enough then we have $R_{z} \leq C_{1}+C_{2}$ for all $z \in \Omega$, and if $e^{2 v}|d z|^{2}$ is the hyperbolic metric on $\Omega$ then $w=\log \left|\partial_{z} h\right| \geq v-C$ for some constant $C$ for all $z \in \partial \Omega_{1}$. Here we have used the fact that the hyperbolic metric on $\Omega$ is dominated by the hyperbolic metric on its subdomain.

Next we want to show that $\inf _{z \in \mathbb{C} \backslash \Omega_{1}} R_{z}>0$. In fact, if $z \in \mathbb{C} \backslash \Omega_{1}$, then there is $k$ such that $\Re \zeta_{k}(z) \geq b$. Apply Corollary 2.1 on the disk with center $\zeta_{k}$ and radius 1 , we can conclude that on $\Re \zeta_{k} \geq b$ the maximal $\Phi$ radius is bounded below by a positive constant independent of $\zeta_{k}$, because $\Phi=\exp \left(\zeta_{k}+o(1)\right) d \zeta_{k}^{2}$.

Since $\Omega$ is strongly hyperbolic and $|\partial h|^{2}|d z|^{2}$ is complete in $\mathbb{C}, h$ is quasiconformal on $\Omega_{1}$ by Theorem 2.1 and Remark 2.1.

On the other hand, by Lemma 3.1, if we choose $a$ and $b$ large enough, then for each $k$, there exist $p_{j}^{(k)} \in \partial \mathbb{H}^{2}, j \in \mathbb{Z}$, which are monotone in $\mathbb{S}^{1}$ 
such that the intersection of the closure of the image under $h$ of the set $\left\{\zeta \in \mathcal{R}_{2}, \Re \zeta \geq b\right\}$ with $\partial \mathbb{H}^{2}$ is equal to

$$
\mathcal{A}=\bigcup_{k=0}^{n-1}\left\{p_{j}^{(k)} \mid j \in \mathbb{Z}\right\} \cup \bigcup_{k=0}^{n-1}\left\{p_{+}^{(k)}, p_{-}^{(k)}\right\},
$$

where $p_{ \pm}^{(k)}=\lim _{j \rightarrow \pm \infty} p_{j}^{(k)}$. Moreover, if $\zeta_{n} \in \mathcal{R}_{2}$ with $\Re \zeta_{n} \geq b$ and $\Im z_{n} \rightarrow$ $+\infty$ (respectively $\Im z_{n} \rightarrow-\infty$ ) then $h\left(\zeta_{n}\right) \rightarrow p_{+}^{(k)}$ (respectively $h\left(\zeta_{n}\right) \rightarrow$ $\left.p_{-}^{(k)}\right)$.

Since $h$ is at most linear growth in $\mathcal{R}_{2}$ with respect to $\zeta, h$ is of polynomial growth on $V_{k, \epsilon}$, provided $\epsilon>0$ is small enough. It is easy to see that $h$ is at most of linear growth on $W_{k}$. By the definition of $\Omega_{1}$, we see that $h$ is of polynomial growth on $\Omega_{1}$. Namely, there exist positive constants $\ell$ and $C$ such that

$$
d_{\mathbb{H}^{2}}(h(z), o) \leq C\left(d_{\mathbb{C}}(z, 0)+1\right)^{\ell}
$$

for all $z \in \Omega_{1}$, where $o$ is a fixed point in $\mathbb{H}^{2}$ and 0 is the origin of $\mathbb{C}$. We claim that the image of $h$ is the convex hull of $\mathcal{A}$ together with at most finitely many points $q_{j} \in \partial \mathbb{H}^{2}$. By theorem 4.8 in [C-T] and theorem 5 in [Wn], it is sufficient to show that $\overline{h(\mathbb{C})} \cap \partial \mathbb{H}^{2}$ is $\left\{p_{j}^{(k)} \mid j \in \mathbb{Z}\right\} \cup\left\{p_{+}^{(k)}, p_{-}^{(k)}\right\}$ together with at most finitely many points $q_{j}$. Suppose $q_{1}, \ldots, q_{m}$ are distinct points in

$$
\left(\overline{h(\mathbb{C})} \cap \partial \mathbb{H}^{2}\right) \backslash \mathcal{A} .
$$

There exist disjoint neighborhoods $U_{1}, \ldots, U_{m}$ of $q_{1}, \ldots, q_{m}$ respectively in $\overline{\mathbb{H}}^{2}$. We may choose $U_{j}, 1 \leq j \leq m$ small enough so that $h^{-1}\left(U_{j}\right) \subset \Omega_{1}$. For if this is not true, then there exists $q_{j}$ and a sequence of neighborhoods $U_{j, n}$ such that $\bigcap_{n=1}^{\infty} U_{j, n}=\left\{q_{j}\right\}$ and such that $h^{-1}\left(U_{j, n}\right)$ is not contained in $\Omega_{1}$ for each $n$. By choosing a subsequence, we may assume that there is $z_{n} \in U_{j, n}$ such that $\Re \zeta_{k}\left(z_{n}\right) \geq b$ under the map $\zeta_{k}$ described above. Since $h\left(z_{n}\right) \rightarrow q_{j}$ by construction, we conclude that $q_{j}$ must be $p_{l}^{(k)}$ or $p_{ \pm}^{k}$ for some $k$ and $l$. This is a contradiction. Hence we may choose $U_{j}$ such that $h^{-1}\left(U_{j}\right)$ is contained in $\Omega_{1}$. Moreover, we may assume that $U_{j}$ is bounded by a geodesic line in $\mathbb{H}^{2}$. Let $f_{j}(z)=d_{\mathbb{H}^{2}}\left(u(z), \mathbb{H}^{2} \backslash U_{j}\right)$, then $f_{j}$ subharmonic because $d_{\mathbb{H}^{2}}\left(\cdot, \mathbb{H}^{2} \backslash U_{j}\right)$ is convex by [B-O]. Note that $f_{j}$ is smooth in $h^{-1}\left(U_{j}\right)$, $f_{j}(z)=0$ for $z \in \mathbb{C} \backslash h^{-1}\left(U_{j}\right)$ and there exists a constant $C_{3}$

$$
f_{j}(z) \leq C_{3}\left(d_{\mathbb{C}}(z)+1\right)^{\ell}
$$


for all $z$ and for all $1 \leq j \leq m$ by (3.3). Since $h^{-1}\left(U_{j}\right), 1 \leq j \leq m$, are disjoint and nonempty, $m$ is bounded from above by a constant depending only on $\ell$ by Theorem 3.4 in [L-W1]. This proves the claim.

Observe that each $q_{j}$ must lie between $p_{+}^{(k)}$ and $p_{-}^{(k+1)}$ for some $k$. Here we use the convention that $p_{+}^{(n)}=p_{-}^{(0)}$. Since if $\gamma_{k}(t)=\zeta^{-1}(b+\sqrt{-1} t)$, then $\lim _{t \rightarrow \pm \infty} h\left(\gamma_{k}(t)\right)=p_{k, \pm}$, we conclude that $h\left(\Omega_{1}\right)$ is bounded by $h\left(\gamma_{k}\right)$ and the geodesics joining consecutive points of $p_{+}^{k}, q_{j}$ and $p_{-}^{(k+1)}$, with $q_{j}$ between $p_{+}^{k}$ and $p_{-}^{(k+1)}$, and they are oriented positively. Since $h$ is quasi-conformal on $\Omega_{1}$, for each $k$ if $\zeta_{n} \in \mathcal{R}_{1}$ with $\Re \zeta_{n} \leq b$ and $\Im z_{n} \rightarrow+\infty$ (respectively $\left.\Im z_{n} \rightarrow-\infty\right)$ then $h\left(\zeta_{n}\right) \rightarrow p_{+}^{(k)}$ (respectively $h\left(\zeta_{n}\right) \rightarrow p_{-}^{(k)}$ ). Again, using the fact that $h$ is quasi-conformal on $W_{k}$, we conclude that for $z \in W_{k}$, and if $z \rightarrow \infty$ then $h(z)$ will converge to a point $q_{k}$ in $\overline{\mathbb{H}^{2}}$. But $q_{k}$ must be equal to $p_{+}^{(k)}$ and $p_{-}^{(k+1)}$ at the same time. Hence the closure of the image of $h$ is the convex hull of the set $A$ consisting of $p_{j}^{(k)}, q_{k}$ which is countable and has exactly $n$ accumulation points.

It is clear that the theorem is true for any polynomial $Q$ without requiring the leading coefficient to be 1 as long as the zeros of the entire function $P$ are distributed in the corresponding sections. For instance, we conclude immediately from the theorem the following.

Corollary 3.1. Let $h$ be an orientation preserving harmonic diffeomorphism from $\mathbb{C}$ into $\mathbb{H}^{2}$ with Hopf differential $\Phi=P \exp (Q) d z^{2}$ where $P$ and $Q$ are polynomials with $\operatorname{deg} Q=n$. Suppose $|\partial h|^{2}|d z|^{2}$ is complete in $\mathbb{C}$. Then the image of $h$ is the convex hull of a countable set $A$ of $\partial \mathbb{H}^{2}$ with exactly $n$ accumulation points.

Figures 1, 5, and 6 show the horizontal trajectories structures of holomorphic quadratic differentials which are included in the corollary 3.1. Figure 1 also shows the image of the harmonic map corresponding to $e^{z} d z^{2}$ which is the basis of all the discussion in this paper.

\section{Images of harmonic diffeomorphisms with Hopf differentials $f\left(e^{z}\right) d z^{2}$.}

In this section, we will study the images of certain harmonic diffeomorphisms from $\mathbb{C}$ into $\mathbb{H}^{2}$ with Hopf differentials of the form $f\left(e^{z}\right) d z^{2}$. As before for $\alpha \geq 0$, let $\mathbb{C}_{\alpha}=\{z=x+\sqrt{-1} y \mid x>\alpha\}$. 
Lemma 4.1. Let $h: \mathbb{C}_{0} \rightarrow \mathbb{H}^{2}$ be an orientation preserving harmonic diffeomorphic injection with Hopf differential $\Phi=d z^{2}$. Suppose that for some $x_{0}>0, \lim _{y \rightarrow+\infty} h\left(x_{0}+\sqrt{-1} y\right)=p_{1}$ and $\lim _{y \rightarrow-\infty} h\left(x_{0}+\sqrt{-1} y\right)=p_{2}$ for some $p_{1}, p_{2}$ in $\partial \mathbb{H}^{2}$. Then $p_{1}=p_{2}=p$, and for all $x_{0}>0$

$$
\lim _{\substack{|z| \rightarrow \infty \\ \Re z \geq x_{0}}} h(z)=p .
$$

Proof. By proposition 1.5 in [T-W1], see also [Wn], the energy density of $h$ in the half-plane $0<\Re z<\infty$ is bounded. Since for some $x_{0}>0$, $\lim _{y \rightarrow+\infty} h\left(x_{0}+\sqrt{-1} y\right)=p_{1}$ and $\lim _{y \rightarrow-\infty} h\left(x_{0}+\sqrt{-1} y\right)=p_{2}$, where $p_{1}, p_{2} \in \partial \mathbb{H}^{2}$, we conclude that for all $x_{1}>x_{0}>0$,

$$
\lim _{\substack{\Im z \rightarrow+\infty \\ x_{0}<\Re z<x_{1}}} h(z)=p_{1}
$$

and

$$
\lim _{\substack{\Im z \rightarrow-\infty \\ x_{0}<\Re z<x_{1}}} h(z)=p_{2} .
$$

Identify $\overline{\mathbb{H}^{2}}=\mathbb{H}^{2} \cup \partial \mathbb{H}^{2}$ with the unit disk. We claim that for any $x_{0}>0$, the closure of $h\left(\mathbb{C}_{x_{0}}\right)$ in $\overline{\mathbb{H}^{2}}$ is $\bar{\Omega}$ where $\Omega$ is the domain bounded by the curve $h\left(x_{0}+\sqrt{-1} y\right),-\infty<y<\infty$ and one of the arc on $\mathbb{S}^{1}$ with end points $p_{1}$ and $p_{2}$. Obviously, $h\left(\mathbb{C}_{x_{0}}\right)$ is contained in such an $\Omega$ because $h$ is injective. Suppose that the claim is not true, then there is $q$ on the boundary of $h\left(\mathbb{C}_{x_{0}}\right)$ such that $q \in \mathbb{H}^{2}$ and there is a geodesic arc $\gamma$ in $h\left(\mathbb{C}_{x_{0}}\right) \subset \mathbb{H}^{2}$ from a point $q_{1}$ in $h\left(\mathbb{C}_{x_{0}}\right)$ to $q$ with $\gamma(\ell)=q$, where $\ell$ is the length of $\gamma$ and is finite. Without loss of generality, we may assume that $\gamma([0, \ell)) \subset h\left(\mathbb{C}_{x_{0}}\right)$. Let $\beta=h^{-1}(\gamma)$. Then $\beta$ is a path in $\mathbb{C}_{x_{0}}$ such that $\beta(t) \rightarrow \infty$ as $t \rightarrow \ell$ because $q$ is on the boundary of $h\left(\mathbb{C}_{x_{0}}\right)$. Moreover, $\Re \beta(t) \rightarrow+\infty$. Otherwise, we would have $\beta(t) \rightarrow p_{1}$ or $p_{2}$. However, the pull-back metric under $h$ is given by $(e+2) d x^{2}+(e-2) d y^{2}$, where $e$ is the energy density of $h$, and $e>2$. We then have

$$
\begin{aligned}
\ell & =\int_{0}^{\ell}\left[(e+2)\left(\frac{d x}{d t}\right)^{2}+(e-2)\left(\frac{d y}{d t}\right)^{2}\right]^{\frac{1}{2}} d t \\
& \geq \sqrt{2}\left(\lim _{t \rightarrow \ell} x(\ell)-x(0)\right) \\
& =\infty
\end{aligned}
$$


which is a contradiction. Hence $h\left(\mathbb{C}_{x_{0}}\right)=\Omega$. Suppose $p_{1} \neq p_{2}$, then $\overline{h\left(\mathbb{C}_{x_{0}}\right)}$ contains a nontrivial arc on $\mathbb{S}^{1}$. However, for $x_{0}>0, h$ is of at most linear growth. By theorem 3.4 in [L-W1], we conclude that $\overline{h\left(\mathbb{C}_{x_{0}}\right)} \cap \partial \mathbb{H}^{2}$ consists of only finitely many points. Hence we must have $p_{1}=p_{2}=p$. Since $h$ is a diffeomorphism, we must have

$$
\lim _{\substack{|z| \rightarrow \infty \\ \Re z \geq x_{0}}} h(z)=p
$$

Lemma 4.2. Let $0<\beta \leq \pi$ and let $h: e^{\sqrt{-1} \beta} \mathbb{C}_{0} \rightarrow \mathbb{H}^{2}$ be an orientation preserving harmonic diffeomorphic injection with Hopf differential $\Phi=d z^{2}$. Suppose that for some $x_{0}>0$,

$$
\begin{aligned}
& \lim _{y \rightarrow \infty} h\left(e^{\sqrt{-1} \beta}\left(x_{0}+\sqrt{-1} y\right)\right)=p_{1} \quad \text { and } \\
& \lim _{y \rightarrow-\infty} h\left(e^{\sqrt{-1} \beta}\left(x_{0}+\sqrt{-1} y\right)\right)=p_{2}
\end{aligned}
$$

for some $p_{1}, p_{2}$ in $\partial \mathbb{H}^{2}$. Then $p_{1} \neq p_{2}$ and for all $x_{0}>0, \overline{h\left(e^{\sqrt{-1} \beta} \mathbb{C}_{x_{0}}\right)} \cap$ $\partial \mathbb{H}^{2}=\left\{p_{1}, p_{2}\right\}$.

Proof. As in the proof of Lemma 4.1, we conclude that for any $x_{0}>0$,

$$
\lim _{y \rightarrow \infty} h\left(e^{\sqrt{-1} \beta}\left(x_{0}+\sqrt{-1} y\right)\right)=p_{1},
$$

and

$$
\lim _{y \rightarrow-\infty} h\left(e^{\sqrt{-1} \beta}\left(x_{0}+\sqrt{-1} y\right)\right)=p_{2} .
$$

Let $x_{0}>0$. Since $0<\beta \leq \pi$, by Lemma 1.1, suppose $z_{n} \in e^{\sqrt{-1} \beta} \mathbb{C}_{x_{0}}$, if $\Re z_{n} \rightarrow-\infty$, then $\lim _{n \rightarrow \infty} h\left(z_{n}\right)=p_{1}$; and if $\Re z_{n} \rightarrow \infty$, then $\lim _{n \rightarrow \infty} h\left(z_{n}\right)=p_{2}$. If $z_{n} \rightarrow \infty$ and for all $n, x_{0} \leq \Re z_{n}<x_{1}$ for some $x_{1}$, then $h\left(z_{n}\right)$ are uniformly bounded. Hence $\overline{h\left(e^{\sqrt{-1}} \mathbb{C}_{x_{0}}\right)} \cap \partial_{\infty} \mathbb{H}^{2}=\left\{p_{1}, p_{2}\right\}$.

To prove that $p_{1} \neq p_{2}$. Note that $\sqrt{-1} n \in e^{\sqrt{-1} \beta}$ for any positive integer $n$. Moreover, it is easy to see that $z_{n}=e^{\sqrt{-1} \frac{\beta}{3}} \sqrt{-1} n=-n \sin \frac{\beta}{3}+$ $\sqrt{-1} n \cos \frac{\beta}{3}$ and $\tilde{z}_{n}=n+\sqrt{-1} n \cos \frac{\beta}{3}$ are in $e^{\sqrt{-1} \beta} C_{x_{0}}$ if $n$ is large. Let $L_{n}$ be the horizontal line joining $z_{n}$ and $\tilde{z}_{n}$. By the arguments in section 3 of [HTTW], we conclude that $h\left(L_{n}\right)$ is of uniformly bounded distance from the geodesic passing through $h\left(z_{n}\right)$ and $h\left(\tilde{z}_{n}\right)$. Since $h\left(z_{n}\right) \rightarrow p_{1}$ and 
$h\left(\tilde{z}_{n}\right) \rightarrow p_{2}$, if $p_{1}=p_{2}=p$ then $h\left(L_{n}\right) \rightarrow p$ as $n \rightarrow \infty$. On the other hand, $h\left(\sqrt{-1} n \cos \frac{\beta}{3}\right)$ are uniformly bounded. This is a contradiction. Therefore, $p_{1} \neq p_{2}$.

Theorem 4.1. Let $m, n$ be nonnegative intergers and let $P(t)$ be a nonconstant rational function of the form

$$
P(t)=\sum_{k=-m}^{n} a_{k} t^{k},
$$

with $a_{-m} \neq 0 \neq a_{n}$. Suppose $h$ is an orientation preserving harmonic diffeomorphism from $\mathbb{C}$ into $\mathbb{H}^{2}$ with Hopf differential given by

$$
\Phi=P\left(e^{z}\right) d z^{2}
$$

such that $|\partial h|^{2}|d z|^{2}$ is a complete metric. Then $\mathcal{A}=\overline{h(\mathbb{C})} \cap \partial \mathbb{H}^{2}$ is countable which has exactly one accumulation point if $m$ or $n=0$, and $a_{0} \geq 0$; and has two accumulation points otherwise. Moreover $\overline{h(\mathbb{C})}$ is the convex hull of $\mathcal{A}$.

Remark. Figures 1 and 2 in the appendix show horizontal trajectories structures for the case that $m$ or $n=0$, and $a_{0} \geq 0$. In fact, in both figures, $m=0$, and $a_{0}=0$ and 1 respectively. The other case are showed by the Figures 3 and 4 . In Figure $3, m=0$ but $a_{0}=-1$. The image of the corresponding harmonic map has 2 accumulations both are limits from one side. In Figure 4, both $m$ and $n$ are not zero and the image of the corresponding harmonic map has 2 accumulations both are limits from two sides.

Proof. Suppose that $m>0$ and $n>0$. By the proof of Lemma 3.1, we can conclude that there exist $p_{k}, k \in \mathbb{Z}$ such that $\overline{h(\{z \mid \Re z \geq 0\}} \cap \partial \mathbb{H}^{2}$ is equal to $\overline{\left\{p_{k}\right\}_{k \in \mathbb{Z}}}$ and the $p_{k}$ are monotone on $\mathbb{S}^{1}$. Moreover, if $p_{k} \rightarrow p_{ \pm}$as

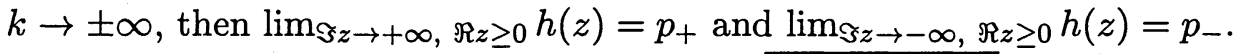

Similarly, there exist $q_{k}, k \in \mathbb{Z}$ such that $\overline{h(\{z \mid \Re z \leq 0\}} \cap \partial \mathbb{H}^{2}$ is equal to $\overline{\left\{q_{k}\right\}_{k \in \mathbb{Z}}}$ and the $q_{k}$ are monotone on $\mathbb{S}^{1}$, and if $q_{k} \rightarrow q_{ \pm}$as $k \rightarrow \pm \infty$, then $\lim _{\Im z \rightarrow+\infty} \Re, \Re_{z \leq 0} h(z)=q_{+}$and $\lim _{\Im z \rightarrow-\infty}, \Re z \leq 0 h(z)=q_{-}$. Hence $q_{+}=p_{+}$and $q_{-}=p_{-}$. Since $h$ is a diffeomorphism, $p_{+} \neq p_{-}$and $\mathcal{A}=$ $\left\{p_{k}, q_{k}\right\}_{k \in \mathbb{Z}} \cup\left\{p_{+}, p_{-}\right\}$, which has two accumulation points.

Next, let us consider the case that $m$ or $n=0$. Without loss of generality, we may assume that $m=0$. As before, there exist $p_{k}, k \in \mathbb{Z}$ such that 
$\overline{h(\{z \mid \Re z \geq 0\}} \cap \partial \mathbb{H}^{2}$ is equal to $\overline{\left\{p_{k}\right\}_{k \in \mathbb{Z}}}$ and the $p_{k}$ are monotone on $\mathbb{S}^{1}$. Let $p_{+}$and $p_{-}$defined as above.

Suppose $a_{0}=0$. Then we can conclude as in the proof of Theorem 3.1 that $p_{+}=p_{-}=p$ and $\mathcal{A}=\left\{p_{k}\right\}_{k \in \mathbb{Z}} \cup\{p\}$ which has only one accumulation point.

Suppose $m=0$ and $a_{0} \neq 0$, let $a_{0}=\rho^{2} e^{2 \sqrt{-1} \beta}$ with $0 \leq \beta<\pi, \rho>0$. There exists $\delta>0$ such that if $|t|<\delta$, we can take a branch of the square root of $P(t)$ and

$$
\sqrt{P(t)}=\rho e^{\sqrt{-1} \beta}+t g(t)
$$

where $g(t)$ is analytic and

$$
|g(t)| \leq C_{1}
$$

for some constant $C_{1}$ for $|t|<\delta$. Let $\tilde{g}(t)$ be such that $\tilde{g}^{\prime}=g$ on $|t|<\delta$ and $\tilde{g}(0)=0$. Let $x_{0}<0$ be small enough so that $\left|e^{z}\right|<\delta$ on $\Re z \leq x_{0}$. Define

$$
\begin{aligned}
\zeta(z) & =\int_{x_{0}}^{z} \sqrt{P\left(e^{\xi}\right)} d \xi \\
& =\rho e^{\sqrt{-1} \beta}\left(z-x_{0}\right)+\int_{x_{0}}^{z} e^{\xi} g\left(e^{\xi}\right) d \xi \\
& =\rho e^{\sqrt{-1} \beta} z+\tilde{g}\left(e^{z}\right)+\zeta_{0}
\end{aligned}
$$

for all $z$ with $\Re z \leq x_{0}$, where $\zeta_{0}$ is a constant. Here the integration is along the straight line from $x_{0}$ to $z$. Then $\zeta$ is analytic. By (4.1), if we choose $x_{0}$ small enough, then $\zeta$ is injective. Since $\left|\tilde{g}\left(e^{z}\right)\right| \leq C_{2}\left|e^{z}\right|$ for some constant $C_{2}$, if we choose $x_{0}$ small enough, then the analytic map $z \rightarrow \zeta_{1}=-\left(\zeta-\zeta_{0}\right)$ will map $\left\{\Re z \leq x_{0}\right\}$ injectively onto its image $\mathcal{R}$. Moreover

$$
e^{\sqrt{-1} \beta}\left\{\zeta_{1} \mid \Re z_{1} \geq \rho x_{0}+1\right\} \subset \mathcal{R} \subset e^{\sqrt{-1} \beta}\left\{\zeta_{1} \mid \Re z_{1} \geq \rho x_{0}-1\right\} .
$$

The Hopf differential of $h$ in the $\zeta_{1}$ plane is given by $d \zeta_{1}^{2}$. As before, we have

$$
\lim _{y \rightarrow \pm \infty} h\left(x_{0}+\sqrt{-1} y\right)=p_{ \pm} \text {. }
$$

Hence if $\beta=0$, we have $p_{+}=p_{-}=p$ by Lemma 4.1 and $\mathcal{A}$ is countable with only one accumulation point. If $\beta>0$, we have $p_{+} \neq p_{-}$by Lemma 4.2 and $\mathcal{A}$ is countable with exactly two accumulation points. The last statement of the theorem follows from Theorem 4.8 in [C-T] and Theorem 5 in [Wn].

As an application, we use Theorem 4.1 to study harmonic diffeomorphic injection from a flat cylinder to a hyperbolic cylinder. Let $N$ be a hyperbolic 
cylinder and let $\mathbb{C}^{*}=\mathbb{C} \backslash\{0\}$. Let $\Phi\left(\mathbb{C}^{*}, N\right)$ be the set of all Hopf differentials of orientation preserving harmonic diffeomorphic injections $h$ from $\mathbb{C}^{*}$ to $N$ such that $|\partial h|^{2}|d z|^{2}$ is complete on $\mathbb{C}^{*}$. Let $\mathcal{P}(N)$ be the set of holomorphic quadratic differentials on $\mathbb{C}^{*}$ defined by

$$
\begin{gathered}
\mathcal{P}(N)=\left\{\frac{P(z)}{z^{2}} d z^{2} \mid P(z)=\sum_{k=-m}^{n} a_{k} z^{k}\right. \\
\text { for some } \left.0 \leq m, n \in \mathbb{Z}, \text { and } P \neq a_{0}\right\}, \\
\mathcal{P}_{1}(N)=\left\{\frac{P(z)}{z^{2}} d z^{2} \in \mathcal{P}(N) \mid P(z)=\sum_{k=-m}^{n} a_{k} z^{k}\right. \\
\text { with } \left.m \text { or } n=0, \text { and } a_{0} \geq 0\right\}
\end{gathered}
$$

and $\mathcal{P}_{2}(N)=\mathcal{P}(N) \backslash \mathcal{P}_{1}(N)$.

Corollary 4.1. With the above notations we have $\Phi\left(\mathbb{C}^{*}, N\right) \cap \mathcal{P}(N)$ is either a subset of $\mathcal{P}_{1}(N)$ or a subset of $\mathcal{P}_{2}(N)$. Moreover, if $\Phi\left(\mathbb{C}^{*}, N\right) \cap \mathcal{P}(N) \neq \emptyset$, then it is a subset of $\mathcal{P}_{1}(N)$ if and only if $N$ has a cusp.

Proof. Let $z^{-2} P(z) d z^{2} \in \Phi\left(C^{*}, N\right)$ be the Hopf differential of an orientation preserving harmonic diffeomorphic injections $h$ from $\mathbb{C}^{*}$ into $N$. Lifting $h$ to the universal coverings, we have an orientation preserving harmonic diffeomorphic injection, denoted by $h$ again, from $\mathbb{C}$ into $\mathbb{H}^{2}$, with Hopf differential given by

$$
P\left(e^{z}\right) d z^{2}
$$

and an element $\rho$ of the Möbius group which generates $\pi_{1}(N)$ such that

$$
h(z+2 \pi i)=\rho(h(z)) .
$$

Note that $|\partial h|^{2}|d z|^{2}$ is complete on $\mathbb{C}$. Let $\mathcal{A}=\overline{h(\mathbb{C})} \cap \partial_{\infty} \mathbb{H}^{2}$. Since $h$ is equivariant, $\mathcal{A}$ is invariant under $\rho$. This implies that the set of fixed points of $\rho$ is exactly the set of accumulation points of $A$. The corollary then follows easily from Theorem 4.1 .

Remark 4.1. It was proved in [Wn, W-A, T-W1] that given a holomorphic quadratic differential $\Phi$ on $\mathbb{C}$ or on $\mathbb{H}^{2}$ there exists an orientation preserving harmonic diffeomorphic injection from $\mathbb{C}$ or $\mathbb{H}^{2}$ to $\mathbb{H}^{2}$ whose Hopf differential 
is the given $\Phi$. Corollary 4.1 shows that the prescribed Hopf differential problem is not alway solvable from $\mathbb{C}^{*}$ into $N$ where $N$ is a hyperbolic cylinder.

Our next result is to consider the image of a harmonic map with Hopf differential of infinite order.

Theorem 4.2. Let $h$ be an orientation preserving harmonic diffeomorphic injection from $\mathbb{C}$ into $\mathbb{H}^{2}$ such that $|\partial h|^{2}|d z|^{2}$ is complete. Suppose that the Hopf differential of $h$ is given by

$$
\Phi=\exp ^{(k)}(z) d z^{2}
$$

for some positive integer $k$, where $\exp ^{(k)}(z)$ is defined inductively by $\exp ^{(0)}(z)=1$ and $\exp ^{(j)}(z)=\exp \left(\exp ^{(j-1)}(z)\right)$. Let $\mathcal{A}=\overline{h(\mathbb{C})} \cap \partial \mathbb{H}^{2}$. Then $\mathcal{A}=\cup_{j=0}^{k} \mathcal{A}_{j}$ such that

- $A_{j}$ is countable and discrete for each $0 \leq j \leq k-1$;

- $\mathcal{A}_{j}$ consists of all isolated accumulation points of $\mathcal{A}_{j-1}$ for $1 \leq j \leq k$;

- $\mathcal{A}_{k}$ consists of only one point.

Please see Figure 7 in the appendix for the horizontal trajectories structure of $e^{e^{z}} d z^{2}$ and the corresponding image of the harmonic map.

Proof. We may assume that $k \geq 2$ because $k=1$ is a special case of Theorem 3.1. First of all, we want to find out the domains such that $\Phi$ can be written in the form of Lemma 3.1. Given any $\alpha \in \mathbb{R}, 1 \leq l \leq k-1$ and $\left(n_{1}, n_{2}, \ldots, n_{l}\right) \in \mathbb{Z}^{l}$, we define the open subsets $\mathcal{S}_{\left(n_{1}, \ldots, n_{l}\right)}$ inductively by

$$
\mathcal{S}_{\left(n_{1}\right)}=\left\{z \in \mathbb{C}|\Re z>\alpha,| \Im z-2 n_{1} \pi \mid<\pi\right\}
$$

and

$$
\mathcal{S}_{\left(n_{1}, \ldots, n_{l}\right)}=\left\{z \in \mathcal{S}_{\left(n_{1}, \ldots, n_{l-1}\right)}\left|\Re \zeta_{l-1}>\exp ^{(l-1)}(\alpha),\right| \Im \zeta_{l-1}-2 n_{l} \pi \mid<\pi\right\},
$$

where $\zeta_{l-1}=\exp ^{(l-1)}(z)$. Then $\zeta_{l}=e^{\zeta_{l-1}}=\exp ^{(l)}(z)$ maps $\mathcal{S}_{\left(n_{1}, \ldots, n_{l}\right)}$ oneone onto the open set

$$
\Omega_{l}=\mathbb{C} \backslash\left(\left\{\zeta_{l} \in \mathbb{R} \mid \zeta_{l} \leq 0\right\} \cup\left\{\zeta_{l}|| \zeta_{l} \mid \leq \exp ^{(l)}(\alpha)\right\}\right),
$$


and in terms of $\zeta_{l}$

$$
\Phi=\frac{\exp ^{(k-l)}\left(\zeta_{l}\right)}{\prod_{j=0}^{l-1}\left(\log ^{(j)} \zeta_{l}\right)^{2}} d \zeta_{l}^{2}
$$

In particular, for $l=k-1$,

$$
\begin{aligned}
\Phi & =\frac{\exp \left(\zeta_{k-1}\right)}{\prod_{j=0}^{k-2}\left(\log ^{(j)} \zeta_{k-1}\right)^{2}} d \zeta_{k-1}^{2} \\
& =\exp \left(\zeta_{k-1}-2 \sum_{j=1}^{k-1} \log ^{(j)} \zeta_{k-1}\right) d \zeta_{k-1}^{2}
\end{aligned}
$$

on

$$
\Omega_{k-1}=\mathbb{C} \backslash\left(\left\{\zeta_{k-1} \in \mathbb{R} \mid \zeta_{k-1} \leq 0\right\} \cup\left\{\zeta_{k-1}|| \zeta_{k-1} \mid \leq \exp ^{(k-1)}(\alpha)\right\}\right) .
$$

A further transformation

$$
\eta=\zeta_{k-1}-2 \sum_{j=1}^{k-1} \log ^{(j)} \zeta_{k-1}
$$

will put the Hopf differential into the form of Lemma 3.1 and we can conclude on the boundary behaviour of the harmonic map $h$. However, to ensure that there are no other ideal boundary point, we need to show that $h$ is quasiconformal in certain domain.

In order to do so, given any $\beta \gg 1$, we define $E_{\beta}=\left\{\zeta_{k-1} \in\right.$ $\left.\Omega_{k-1} \mid \Re \eta\left(\zeta_{k-1}\right)>\beta\right\}$ and claim that for any $\alpha \in \mathbb{R}$, there are simplyconnected domains $V_{0} \subset \tilde{V}_{0} \subset\{z \in \mathbb{C} \mid \Re z>\alpha-1\}$ such that

$$
\mathbb{C} \backslash \tilde{V}_{0}=\{\Re z<\alpha-1\} \cup\left(\bigcup_{\left(n_{1} \ldots, n_{k-1}\right)} \tilde{T}_{\left(n_{1} \ldots, n_{k-1}\right)}\right)
$$

and

$$
\mathbb{C} \backslash V_{0}=\{\Re z<\alpha\} \cup\left(\bigcup_{\left(n_{1} \ldots, n_{k-1}\right)} T_{\left(n_{1} \ldots, n_{k-1}\right)}\right),
$$

where $\tilde{T}_{\left(n_{1} \ldots, n_{k-1}\right)}$ (respectively $\left.T_{\left(n_{1} \ldots, n_{k-1}\right)}\right)$ is the component of the preimage of $E_{\beta+1}$ (respectively $E_{\beta}$ ), under the map $\exp ^{(k-1)}(z)$ corresponding to the 
branch of $\log$ given by $\left(n_{1}, \ldots, n_{k-1}\right)$. Moreover, there are constants $C_{0}$, $M_{0}, \delta$ with $C_{0}$ and $\delta>0$ such that

$$
\sup _{\tilde{V}_{0}} R_{z} \leq C_{0}, \quad \inf _{\mathbb{C} \backslash V_{0}} R_{z} \geq \delta, \quad \text { and } \quad \inf _{\partial V_{0}}(w-v)(z) \geq M_{0},
$$

where $R_{z}$ is the maximal $\Phi$-radius at $z, w=\log |\partial h|$ and $e^{2 v}|d z|^{2}$ is the Poincaré metric on $\tilde{V}_{0}$. If the claim is true, then the last inequality of (4.4) implies that $w \geq v-M_{0}$ for all $z \in V_{0}$, and hence, by Theorem 2.1, one can conclude that $h$ is quasiconformal on $V_{0}$.

To prove the claim, we note that for $z \in T_{\left(n_{1}, \ldots, n_{k-1}\right)}$ the image of $z$

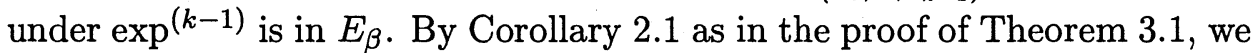
conclude that $R_{z} \geq \delta>0$ for some $\delta>0$ independent of $\left(n_{1}, \ldots, n_{k-1}\right)$. On the other hand, since $e^{(k)}(z) \rightarrow e^{(k-1)}(0)$ if $\Re z \rightarrow-\infty$, we also have $R_{z} \geq \delta$ by Corollary 2.1 if $\Re z \leq \alpha$ and by choosing a possible smaller $\delta$. The second inequality of (4.4) is proved.

Let $z \in \partial V_{0}$, then either $\Re z=\alpha$ or the image of $z$ under $\exp ^{(k-1)}$ is on the boundary of $E_{\beta}$. In the first case, $e^{2 w(z)} \geq\left|e^{(k-1)}(z)\right| \geq C$ for some constant $C>0$ independent of $z$. Hence it is easy to see that $w(z)-v(z) \geq M_{0}$ for some constant $M_{0}$ because $\tilde{V}_{0}$ is strongly hyperbolic. In the second case, we can proceed as in the proof of Theorem 3.1 and obtain the third inequality in (4.4).

To prove the first inequality in (4.4), we let

$$
V_{k-1}=\Omega_{k-1} \backslash E_{\beta} \subset \tilde{V}_{k-1}=\Omega_{k-1} \backslash E_{\beta+1} .
$$

Then it is easy to see that $V_{k-1} \subset \tilde{V}_{k-1}$ are simply-connected domains in $\Omega_{k-1}$ and there is $C_{k-1}$ such that

$$
\sup _{\tilde{V}_{k-1}} R_{z} \leq C_{k-1} .
$$

In fact, for all $z \in \tilde{V}_{k-1}$, there is a divergent path $\gamma$ in $\tilde{V}_{k-1}$ such that

$$
L_{\Phi}(\gamma)<C_{k-1} .
$$

Now, for $l=k-2$, we consider subsets in $\Omega_{k-2}$ containing the preimage of $V_{k-1}$ and $\tilde{V}_{k-1}$ under the exponential map $\zeta_{k-1}=\exp \left(\zeta_{k-2}\right)$. It is clear from the property of the exponential map that

$$
\begin{aligned}
& V_{k-2}=\exp ^{-1}\left(V_{k-1}\right) \cup\left[\left(\overline{V_{k-2}^{+}} \cup \overline{V_{k-2}^{-}}\right) \cap \Omega_{k-2}\right] \\
& \tilde{V}_{k-2}=\exp ^{-1}\left(\tilde{V}_{k-1}\right) \cup\left[\left(\overline{V_{k-2}^{+}} \cup \overline{V_{k-2}^{-}}\right) \cap \Omega_{k-2}\right]
\end{aligned}
$$


are simply-connected domains in $\Omega_{k-2}$ such that

$$
V_{k-2}^{ \pm} \subset V_{k-2} \subset \tilde{V}_{k-2}
$$

where, for $l=1, \ldots, k-1$,

$$
\begin{aligned}
& V_{l-1}^{+}=\left\{z \in \mathcal{S}_{\left(n_{1}, \ldots, n_{l-1}\right)} \mid \Re \zeta_{l-1}<\exp ^{(l-1)}(\alpha), \Im \zeta_{l-1}>0\right\} \\
& V_{l-1}^{-}=\left\{z \in \mathcal{S}_{\left(n_{1}, \ldots, n_{l-1}\right)} \mid \Re \zeta_{l-1}<\exp ^{(l-1)}(\alpha), \Im \zeta_{l-1}<0\right\} .
\end{aligned}
$$

We note that, for $l=1, \ldots, k-1$,

$$
\mathcal{S}_{\left(n_{1}, \ldots, n_{l-1}\right)}=V_{l-1}^{+} \cup V_{l-1}^{-} \cup\left[\cup_{n_{l} \in \mathbb{Z}}\left(\overline{\mathcal{S}_{\left(n_{1}, \ldots, n_{l-1}, n_{l}\right)}} \cap \mathcal{S}_{\left(n_{1}, \ldots, n_{l-1}\right)}\right)\right] .
$$

We want to show that there exists $C_{k-2}^{\prime}>0$ such that for all $z \in \tilde{V}_{k-2}$, there is a divergent path $\gamma$ in $\tilde{V}_{k-2}$ with $L_{\Phi}(\gamma)<C_{k-2}^{\prime}$. This will immediately implies that

$$
\sup _{\tilde{V}_{k-2}} R_{z} \leq C_{k-2}
$$

for some $C_{k-2}>0$. To prove this, we note that for all $r_{0}>\exp ^{(k-2)}(\alpha)$,

$$
\begin{aligned}
\int_{r=r_{0}, 0<\theta<\pi}|\Phi|\left|d \zeta_{k-2}\right| & \leq C \int_{r=r_{0}, 0<\theta<\pi} \frac{r d \theta}{\left|\zeta_{k-2}\right|\left|\log \zeta_{k-2}\right| \cdots\left|\log { }^{(k-2)} \zeta_{k-2}\right|} \\
& \leq \frac{C}{\log r_{0} \cdots \log ^{(k-2)} r_{0}} \\
& \rightarrow 0 \text { as } r_{0} \rightarrow+\infty .
\end{aligned}
$$

Then for all point $\zeta_{k-2} \in V_{k-2}^{ \pm}$, it can be connected to a point on the vertical line $\left\{\Re \zeta_{k-2}=\exp ^{(k-2)}(\alpha)\right\}$ by a circular arc with uniformly bounded $\Phi$ length. By using $\zeta_{k-1}=\exp \left(\zeta_{k-2}\right)$ to map a point on $\partial \Omega_{k-1}$, we can find a divergent path in $\tilde{V}_{k-1}$ with $\Phi$-length bounded by $C_{k-1}$. Lifting this path to $\tilde{V}_{k-2}$ and together with the circular arc, we find a path starting from any point in $V_{k-2}^{ \pm}$a divergent path in $\tilde{V}_{k-2}$. The same is obviously true for other $z \in \tilde{V}_{k-2}$ since they belong to $\exp ^{-1}\left(\tilde{V}_{k-1}\right)$. This proves our assertion that

$$
\sup _{\tilde{V}-2} R_{z} \leq C_{k-2}
$$

for some $C_{k-2}>0$.

Continue in this way, for all $l \leq k-1$ we can define $V_{l}, \tilde{V}_{l}$ and $V_{l}^{ \pm}$, such that

$$
V_{0}=\exp ^{-1}\left(V_{1}\right) \quad \text { and } \tilde{V}_{0}=\exp ^{-1}\left(\tilde{V}_{1}\right) \cup\{z \mid \alpha-1<\Re z<\alpha\} .
$$


Moreover, we can prove inductively that

$$
\sup _{\tilde{V}_{l}} R_{z} \leq C_{l}
$$

for some constant $C_{l}$. Finally, it is easy to see that $R_{z} \leq C$ for some contant $C$ if $\alpha-1<\Re z<\alpha$ because such a point can be joined by a line with bounded $|\phi|$-length to a point in $\exp ^{-1}\left(\tilde{V}_{1}\right)$. This completes the proof of the first inequality in (4.4).

Now we can study the structure of the boundary points of $h(\mathbb{C})$. Firstly, for any $\left(n_{1}, \ldots, n_{k-1}\right) \in \mathbb{Z}^{k-1}$, using Lemma 3.1 , we can argue as before to conclude that there exists monotone sequence $p_{\left(n_{1}, \ldots, n_{k-1}\right) ; j_{0}}, j_{0} \in \mathbb{Z}$ and $p_{\left(n_{1}, \ldots, n_{k-1}\right) ;+}, p_{\left(n_{1}, \ldots, n_{k-1}\right) ;-}$ in $\partial \mathbb{H}^{2}$ such that, for $\beta$ sufficiently large,

$$
\begin{aligned}
& \overline{h\left(\mathcal{S}_{\left(n_{1}, \ldots, n_{k-1}\right)} \cap\{z \mid \Re \eta(z) \geq \beta\}\right)} \cap \partial \mathbb{H}^{2} \\
& =\left\{p_{\left(n_{1}, \ldots, n_{k-1}\right) ; j_{0}}\right\}_{j_{0} \in \mathbb{Z}} \cup\left\{p_{\left(n_{1}, \ldots, n_{k-1}\right) ;+}, p_{\left(n_{1}, \ldots, n_{k-1}\right) ;-}\right\} \\
& \lim _{j_{0} \rightarrow \pm \infty} p_{\left(n_{1}, \ldots, n_{k-1}\right) ; j_{0}}=p_{\left(n_{1}, \ldots, n_{k-1}\right) ;} \cdot
\end{aligned}
$$

Moreover, if $z_{n} \in \mathcal{S}_{\left(n_{1}, \ldots, n_{k-1}\right)} \cap\{z \mid \Re \eta(z) \geq \beta\}$ and $z_{n} \rightarrow \infty$ then

$$
h\left(z_{n}\right) \rightarrow \begin{cases}p_{\left(n_{1}, \ldots, n_{k-1}\right) ;+}, & \text { if } \Im \eta\left(z_{n}\right) \rightarrow \infty \\ p_{\left(n_{1}, \ldots, n_{k-1}\right) ;-}, & \text { if } \Im \eta\left(z_{n}\right) \rightarrow-\infty .\end{cases}
$$

In fact, we conclude by (4.3) that the energy density of $h$ is bounded on the set $z \in \mathcal{S}_{\left(n_{1}, \ldots, n_{k-1}\right)}$ such that $a<\Re \eta(z)<b$ and $|\Im \eta(z)| \geq R$ for any $a, b$ and $R$ provided $R$ is large enough. Hence we still have $h\left(z_{n}\right) \rightarrow p_{\left(n_{1}, \ldots, n_{k-1}\right) ; \pm}$ if $\Im \eta\left(z_{n}\right) \rightarrow \pm \infty, z_{n} \in \mathcal{S}_{\left(n_{1}, \ldots, n_{k-1}\right)}$ and $a<\Re \eta\left(z_{n}\right)<b$.

Secondly, for any $n_{1}, \ldots, n_{k-2} \in \mathbb{Z}^{k-2}$ and for any $j_{1} \in \mathbb{Z}$, the map $\zeta_{k-1}=\exp \left(\zeta_{k-2}\right)$ will map

$$
\left\{z \in \mathcal{S}_{\left(n_{1}, \ldots, n_{k-2}\right)}\left|\Re \zeta_{k-2}(z)>\exp ^{(k-1)}(\alpha),\right| \Im \zeta_{k-2}(z)-\left(2 j_{1}+1\right) \pi \mid<\pi\right\}
$$

one-one onto

$$
\tilde{\Omega}_{k-1}=\mathbb{C} \backslash\left(\left\{\zeta_{k-1} \in \mathbb{R} \mid \zeta_{k-1} \geq 0\right\} \cup\left\{\zeta_{k-1}|| \zeta_{k-1} \mid \leq \exp ^{(k-1)}(\alpha)\right\}\right) .
$$

The corresponding curves given by $\Re \eta=\beta$ in $\mathcal{S}_{\left(n_{1}, \ldots, n_{k-2}, j_{1}\right)}$ and $\mathcal{S}_{\left(n_{1}, \ldots, n_{k-2}, j_{1}-1\right)}$ give us two branches of curve $\gamma_{+}$and $\gamma_{-}$satisfying $\Re \eta=\beta$ on $\tilde{\Omega}_{k-1} \cap\left\{\Im \zeta_{k-1}>0\right\}$ and $\tilde{\Omega}_{k-1} \cap\left\{\Im \zeta_{k-1}<0\right\}$ respectively. Joining the two branches of curve by a compact curve $\gamma$ in $\tilde{\Omega}_{k-1}$, for instance a circular 
arc with sufficiently large radius centered at the origin, gives a subset $U$ with $\partial U=\gamma_{+} \cup \gamma_{-} \cup \gamma$ on which $h$ is quasi-conformal. By (4.6), $h$ will maps $\partial U$ to a curve in $\mathbb{H}^{2}$ such that if $\Im \eta \rightarrow \infty$ the image under $h$ will tends to the point $p_{\left(n_{1}, \ldots, n_{k-2}, j_{1}+1\right) ;-}$, and if $\Im \eta \rightarrow-\infty$ the image under $h$ will tends to the point $p_{\left(n_{1}, \ldots, n_{k-2}, j_{1}\right) ;+}$ As in the proof of Theorem 3.1, we see that $p_{\left(n_{1}, \ldots, n_{k-2}, j_{1}+1\right) ;-}=p_{\left(n_{1}, \ldots, n_{k-2}, j_{1}\right) ;+}$ which will be denoted by $p_{\left(n_{1}, \ldots, n_{k-2}\right) ; j_{1}}$. It is then not hard to see that

$\overline{h\left(S_{\left(n_{1}, \ldots, n_{k-1}\right)}\right)} \cap \partial \mathbb{H}^{2}=\left\{p_{\left(n_{1}, \ldots, n_{k-1}\right) ; j_{0}}\right\}_{j_{0} \in \mathbb{Z}} \cup\left\{p_{\left(n_{1}, \ldots, n_{k-1}\right) ;+}, p_{\left(n_{1}, \ldots, n_{k-1}\right) ;-}\right\}$, and

$$
\mathcal{A}_{0}=\left\{p_{\left(n_{1}, \ldots, n_{k-2}, n_{k-1}\right) ; j_{0}}\right\}_{\left(n_{1}, \ldots, n_{k-1}\right) \in \mathbb{Z}^{(k-1)}, j_{0} \in \mathbb{Z}}
$$

is countable and discrete. Now for each $\left(n_{1}, \ldots, n_{k-2}\right) \in \mathbb{Z}^{(k-1)}$ the set

$$
\left\{p_{\left(n_{1}, \ldots, n_{k-2}\right) ; j_{1}}\right\}_{j_{1} \in \mathbb{Z}}
$$

is monotone in $j_{1}$ and we denote $p_{\left(n_{1}, \ldots, n_{k-2}\right) ; \pm}=\lim _{j_{1} \rightarrow \pm \infty} p_{\left(n_{1}, \ldots, n_{k-2}\right) ; j_{1}}$. Since the Hopf differential on $\mathcal{S}_{\left(n_{1}, \ldots, n_{k-1}\right)}$ is of the same form (4.3), by the proof of Lemma 3.1, for each $\left(n_{1}, \ldots, n_{k-1}\right)$, there is a point $z_{\left(n_{1}, \ldots, n_{k-1}\right)} \in$ $\mathcal{S}_{\left(n_{1}, \ldots, n_{k-1}\right)}$ and two consecutive points in $\left\{p_{\left(n_{1}, \ldots, n_{k-1} ; j_{0}\right)}\right\}_{j_{0} \in \mathbb{Z}}$ such that

$$
\begin{gathered}
\Re \eta\left(z_{\left(n_{1}, \ldots, n_{k-1}\right)}\right)=\beta \\
\left|\Im \eta\left(z_{\left(n_{1}, \ldots, n_{k-1}\right)}\right)\right| \leq \pi,
\end{gathered}
$$

and that the distance from $h\left(z_{\left(n_{1}, \ldots, n_{k-1}\right)}\right)$ to the geodesic joining these two consecutive points is bounded by $C_{1}$ for some constant $C_{1}>0$ which is independent of $\left(n_{1}, \ldots, n_{k-1}\right)$. From (4.7) and (4.8) we have

$$
\lim _{j_{1} \rightarrow \pm \infty} h\left(z_{\left(n_{1}, \ldots, n_{k-2}, j_{1}\right)}\right)=p_{\left(n_{1}, \ldots, n_{(k-2)}\right) ; \pm} \cdot
$$

Using (4.2) and (4.9), we can argue as before to conclude that

$$
p_{\left(n_{1}, \ldots, n_{k-3}, j_{2}+1\right) ;-}=p_{\left(n_{1}, \ldots, n_{k-3}, j_{2}\right) ;+}
$$

which will be denoted by $p_{\left(n_{1}, \ldots, n_{k-3}\right) ; j_{2}}$, and

$$
\overline{h\left(S_{\left(n_{1}, \ldots, n_{k-2}\right)}\right)} \cap \partial \mathbb{H}^{2}=\mathcal{A}_{0} \cup\left\{p_{\left(n_{1}, \ldots, n_{k-3}, n_{k-2}\right) ;+}, p_{\left(n_{1}, \ldots, n_{k-3}, n_{k-2}\right) ;-}\right\} .
$$

Let

$$
\mathcal{A}_{1}=\left\{p_{\left(n_{1}, \ldots, n_{k-2}\right) ; j_{1}}\right\}_{\left(n_{1}, \ldots, n_{k-2}\right) \in \mathbb{Z}^{k-2}, j_{1} \in \mathbb{Z}}
$$


Then $\mathcal{A}_{1}$ is countable and each point in $\mathcal{A}_{1}$ is an isolated accumulation point of $\mathcal{A}_{0}$. The accumulation points of $\mathcal{A}_{1}$ are $p_{\left(n_{1}, \ldots, n_{k-3}\right) ; j_{2}},\left(n_{1}, \ldots, n_{k-3}\right) \in$ $\mathbb{Z}^{(k-2)}$ and $j_{2} \in \mathbb{Z}$. Continue in this way, we can find $\mathcal{A}_{j} \subset \partial \mathbb{H}^{2}, 0 \leq j \leq k$ such that each $\mathcal{A}_{j}$ is countable and discrete for $0 \leq j \leq k-1$ and $\mathcal{A}_{j}$ consists of all isolated accumulation points of $\mathcal{A}_{j-1}$ for $1 \leq j \leq k$. Moreover,

$$
\overline{h(\mathbb{C})} \cap \partial \mathbb{H}^{2}=\cup_{j=0}^{k} \mathcal{A}_{j} .
$$

Finally, We want to prove that $\mathcal{A}_{k}$ consists of only one point. From the proof, we can see that $\mathcal{A}_{k}$ consists of at most two points $p$ and $q$ satisfying

$$
\lim _{y \rightarrow \infty} h(\sqrt{-1} y)=p
$$

and

$$
\lim _{y \rightarrow-\infty} h(\sqrt{-1} y)=q
$$

Since

$$
\exp ^{(k-1)}(t)=\exp ^{(k-1)}(0)+t g(t)
$$

on $|t| \leq 1$, where $g(t)$ is analytic, one can proceed as in the proof of Theorem 4.1 to show that $p=q$ and

$$
\overline{h(\{z \mid \Re z \leq 0\}} \cap \partial \mathbb{H}^{2}=\{p\} .
$$

Hence $\mathcal{A}_{k}$ is a singleton and this completes the proof of the theorem.

Remark 4.2. The Theorem 4.1 is also true for the Hopf differential

$$
\exp ^{(k-1) *}\left(e^{z} d z^{2}\right)=\exp ^{(k)}(z) \prod_{j=1}^{k-1}\left[\exp ^{(j)}(z)\right]^{2} d z^{2}
$$

In fact, the proof is much easier and can be done by induction since the form of the Hopf differential is not change under the $\operatorname{map} \zeta=e^{z}$.

Remark 4.3. The Theorem 4.1 is not necessary true in general. In fact, it becomes very complicated for the general form as in Theorem 1.2. Even for $\Phi=P(z) \exp ^{(k)}(z) d z^{2}$, the Theorem 4.1 need modification. For instance, if $P(z)=\sqrt{-1}$, then the same argument as in the proof of Theorem 4.1 and using Lemma 4.2 instead of Lemma 4.1 on the region $\{z<\alpha\}$, we see that the set $\mathcal{A}_{k}$ consists of two points whether than one. So the best to hope for is that $\mathcal{A}_{k}$ has at most two points for the general form in Theorem 1.2. 


\section{Harmonic diffeomorphisms on hyperbolic plane.}

The result in $\S 2$, in particular Proposition 2.1, can be applied to study a conjecture of Schoen, which says that any quasi-symmetric homeomorphism on $\mathbb{S}^{1}$ can be extended to a unique quasi-conformal harmonic diffeomorphism on $\mathbb{H}^{2}$. The existence part of the conjecture is still open, but there are many partial results, see [Ak, L-T1, L-T2, L-T3, T-W2, S-T-W, H-W, Y]. Schoen's conjecture can be reformulated as follows. Let $\operatorname{BQD}\left(\mathbb{H}^{2}\right)$ be the space of holomorphic quadratic differentials $\Phi$ on $\mathbb{H}^{2}$ such that

$$
|||\Phi|||=\sup _{z \in \mathbb{H}^{2}} \| \Phi||(z)<\infty
$$

where $\|\Phi\|(z)$ is the norm of $\Phi$ at $z$ with respect to the Poincaré metric. In [Wn], the third author proved that for any $\Phi \in \mathrm{BQD}\left(\mathbb{H}^{2}\right)$, there is a unique quasi-conformal harmonic diffeomorphism $u$ on $\mathbb{H}^{2}$ with $\Phi$ as Hopf differential. This defines a map $\mathfrak{B}$ from $\operatorname{BQD}\left(\mathbb{H}^{2}\right)$ to the universal Teichmüller space $\mathcal{T}$ by sending $\Phi$ to the class of quasi-symmetric homeomorphism containing the boundary value of $u$. The existence part of the conjecture of Schoen is equivalent to the surjectivity of the map $\mathfrak{B}$. Let $\mathcal{F}$ be a subset of $\mathrm{BQD}\left(\mathbb{H}^{2}\right)$, we say that $\mathfrak{B}$ is proper on $\mathcal{F}$ if for any $\Phi_{n} \in \mathcal{F}$ with $\left\|\left|\Phi_{n} \|\right| \rightarrow \infty\right.$, we have $\left.d_{\mathcal{T}}\left(\mathfrak{B}\left(\Phi_{n}\right), 0\right)\right) \rightarrow \infty$, where $d_{\mathcal{T}}$ is the Teichmüller metric on $\mathcal{T}$. It is not hard to see that $\mathfrak{B}$ is surjective if $\mathfrak{B}$ is proper on $\operatorname{BQD}\left(\mathbb{H}^{2}\right)$. It is also not hard to see that if $\mathfrak{B}$ is proper on the set of $\Phi \in \mathrm{BQD}\left(\mathbb{H}^{2}\right)$ with $\int_{\mathbb{H}^{2}}\|\Phi\| d v_{\mathbb{H}^{2}}<\infty$ or even on the set $\Phi=\phi d z^{2}$ with $\phi$ to be a polynomial, then $\mathfrak{B}$ is proper on $\mathrm{BQD}\left(\mathbb{H}^{2}\right)$. Here, we identify $\mathbb{H}^{2}$ with $\mathbb{D}$ with the Poincaré metric. For the sake of completeness, we give a proof of this fact below. Denote

$$
\mathcal{F}=\left\{\Phi \in \mathrm{BQD}\left(\mathbb{H}^{2}\right) \mid \int_{\mathbb{H}^{2}}\|\Phi\| d v_{\mathbb{H}^{2}}<\infty\right\}
$$

Note that $\Phi=\phi d z^{2}$, then $\int_{\mathbb{H}^{2}}\|\Phi\| d v_{\mathbb{H}^{2}}=\int_{\mathbb{D}}|\phi| d x d y$.

Proposition 5.1. Let

$$
\mathcal{G}=\left\{\Phi \in \mathcal{F} \mid \Phi=\phi d z^{2}, \phi \text { is a polynomial }\right\} .
$$

Then

(i) If $\mathfrak{B}$ is proper on $\mathcal{G}$, then $\mathfrak{B}$ is proper on $\mathcal{F}$.

(ii) If $\mathfrak{B}$ is proper on $\mathcal{F}$, then $\mathfrak{B}$ is proper on $\mathrm{BQD}\left(\mathbb{H}^{2}\right)$. 
(iii) If $\mathfrak{B}$ is proper on $\mathrm{BQD}\left(\mathbb{H}^{2}\right)$, then $\mathfrak{B}$ is surjective.

In particular, if $\mathfrak{B}$ is proper on $\mathcal{G}$, then $\mathfrak{B}$ is surjective.

Proof. (i) First we prove that if $\mathfrak{B}$ is proper on $\mathcal{G}$, then $\mathfrak{B}$ is proper on $\mathcal{F}$. Let $\Phi_{n} \in \mathcal{F}$ such that $\left\|\mid \Phi_{n}\right\| \| \rightarrow \infty$. Suppose that there is a constant $C_{1}$ such that $d_{\mathcal{T}}\left(\mathfrak{B}\left(\Phi_{n}\right), 0\right) \leq C_{1}$ for all $n$. Since $\mathfrak{B}$ is continuous, there exist $\delta_{n}>0$ such that if $\left\|\left|\Phi_{n}-\Psi \|\right| \leq \delta_{n}\right.$, then $d_{\mathcal{T}}(\mathfrak{B}(\Psi), 0) \leq C_{1}+1$. Hence it is sufficient to prove that $\mathcal{G}$ is dense in $\mathcal{F}$. Let $\Phi=\phi d z^{2} \in \mathcal{F}$, then

$$
\int_{\mathbb{D}}|\phi| d x d y=\int_{\mathbb{H}^{2}}\|\Phi\| d v_{\mathbb{H}^{2}}<\infty .
$$

Apply the mean value inequality on the disk $\mathbb{D}_{z, r}$ with center at $z$ and radius $r=\frac{1}{2}(1-|z|)$, we can conclude that $\| \Phi||(z) \rightarrow 0$ uniformly as $|z| \rightarrow 1$. For $0<R<1$, let $\Phi_{R}(z)=\Phi(R z)$. For any $\epsilon>0$, we can find $1>\delta>0$ such that if $1-\delta \leq|z|<1$ then $\|\Phi\|(z) \leq \frac{1}{2} \epsilon$. Then for $1-\frac{1}{2} \delta \leq|z|<1$ and for $R$ large enough, so that $R|z| \geq 1-\delta$

$$
\left\|\Phi_{R}(z)\right\|=\frac{\left(1-|z|^{2}\right)^{2}}{\left(1-|R z|^{2}\right)^{2}}\|\Phi(R z)\| \leq \frac{1}{2} \epsilon .
$$

On the other hand, for $|z| \leq 1-\frac{1}{2} \delta, \phi_{R}(z) \rightarrow \phi(z)$ uniformly, às $R \rightarrow 1$. Hence we can find $R$ large enough, so that

$$
\left\|\Phi_{R}(z)-\Phi(z)\right\| \leq \epsilon
$$

for all $z \in \mathbb{D}$. Hence ||$\left|\Phi_{R}-\Phi\right| \|_{B Q D} \leq \epsilon$. But $\Phi_{R}$ is analytic on $|z|<\frac{1}{R}$ which is large than 1 . So it can be approximated uniformly on $\mathbb{D}$ by polynomials. This completes the proof of (i)

(ii) We will prove that if $\mathfrak{B}$ is proper on $\mathcal{F}$, then $\mathfrak{B}$ is proper on $\mathrm{BQD}\left(\mathbb{H}^{2}\right)$. Let $\Phi \in \mathcal{F}$ and let $\mathfrak{B}(\Phi)=[f]$ where $f$ is a quasi-symmetric homeomorphism of $\mathbb{S}^{1}$ fixing $1, i,-i$. Let $d_{\mathcal{T}}([f], 0)=C_{1}$. Then there exist diffeomorphisms of $\mathbb{S}^{1} g_{k}$ fixing $1, i,-i$ such that $g_{k} \rightarrow f$ in $C^{\alpha}$ norm for some $1>\alpha>0$ and such that $d_{\mathcal{T}}\left(\left[g_{k}\right], 0\right) \leq C_{2}$ which depends only on $C_{1}$. Moreover, $C_{1} \rightarrow \infty$ if and only if $C_{2} \rightarrow \infty$. These follow from theorem 2 and remark (1) in [D-E]. By theorem 6.4 in [L-T3], see also [T-W2], for each $k$ there exists a unique $\Psi_{k} \in \mathrm{BQD}\left(\mathbb{H}^{2}\right)$ such that $\mathfrak{B}\left(\Psi_{k}\right)=\left[g_{k}\right]$ with $\Psi_{k} \in \mathcal{F}$. By the assumption, we have $\left\|\left|\Psi_{k} \|\right| \leq C_{3}\right.$ for all $k$, where $C_{3}$ depends only on $C_{1}$. Note that $\Psi_{k}$ is the Hopf differential of quasi-conformal harmonic diffeomorphism on $\mathbb{H}^{2}$ with boundary value $g_{k}$. Hence $\Psi_{k}(z) \rightarrow \Phi(z)$ for all $z \in \mathbb{D}$ and so

$$
\limsup _{k \rightarrow \infty}\left\|\left|\Psi_{k}\||\geq\||\Phi|\| \text {. }\right.\right.
$$


From this, it is easy to see that $\mathfrak{B}$ is proper on $\operatorname{BQD}\left(\mathbb{H}^{2}\right)$.

(iii) We will prove that if $\mathfrak{B}$ is proper on $\mathrm{BQD}\left(\mathbb{H}^{2}\right)$, then $\mathfrak{B}$ is surjective. Let $[f]$ be a class of quasi-symmetric homeomorphism on $\mathbb{S}^{1}$ such that $[f]$ is in the closure of $\mathfrak{B}\left(\mathrm{BQD}\left(\mathbb{H}^{2}\right)\right)$. Then there exists $f_{n}$ quasi-symmetric homeomorphisms on $\mathbb{S}^{1}$ fixing $1, i,-i$ such that $f_{n} \rightarrow f$ uniformly, and $\left[f_{n}\right]=\mathfrak{B}\left(\Phi_{n}\right)$. Since $\left[f_{n}\right]$ are uniformly bounded on $\mathcal{T}, \Phi_{n}$ are uniformly bounded in $\operatorname{BQD}\left(\mathbb{H}^{2}\right)$. By theorem 13 in [Wn], the quasi-conformal harmonic diffeomorphisms $u_{n}$ with Hopf differentials $\Phi_{n}$ has complex dilatation $\mu_{n}$ satisfying $\left|\mu_{n}\right| \leq \mu<1$ for some constant $\mu$ independent of $n$. Passing to a subsequence if necessary, $u_{n}$ converges uniformly on $\overline{\mathbb{D}}$ to a quasi-conformal harmonic diffeomorphism on $\mathbb{H}^{2}$ with boundary value $f$. Hence $[f]$ is in $\mathfrak{B}\left(\operatorname{BQD}\left(\mathbb{H}^{2}\right)\right)$. Combine with the theorem 4.1 in [T-W2], we conclude that $\mathfrak{B}$ is surjective.

Proposition 5.2. Let $\Phi_{n} \in \mathrm{BQD}\left(\mathbb{H}^{2}\right)$ satisfying $\int_{\mathbb{H}^{2}}\left\|\Phi_{n}\right\|<\infty$ and $\left\||| \Phi_{n}\right\| \mid \rightarrow \infty$. Suppose for all $k>0$,

$$
\lim _{n \rightarrow \infty} \frac{\int_{U_{n}}\left\|\Phi_{n}\right\| d v_{\mathbb{H}^{2}}}{\int_{\mathbb{H}^{2}}\left\|\Phi_{n}\right\| d v_{\mathbb{H}^{2}}}=0
$$

where

$$
U_{n}=\left\{z \in \mathbb { H } ^ { 2 } \left|\left\|\Phi_{n}\right\|(z) \leq k\left\|\left|\Phi_{n} \|\right|^{\frac{3}{4}}\right\} .\right.\right.
$$

Then $d_{\mathcal{T}}\left(\mathfrak{B}\left(\Phi_{n}\right), 0\right) \rightarrow \infty$.

Proof. Identify $\mathbb{H}^{2}$ with the unit disk $\mathbb{D}$ equipped with the Poincaré metric. Let $\Phi_{n}=\phi_{n} d z^{2}$. Let $\mu_{n, 0}$ be the supremum of the modulus of the complex dilatation of $\mathfrak{B}\left(\Phi_{n}\right)$, then

$$
d_{\mathcal{T}}\left(\mathfrak{B}\left(\Phi_{n}\right), 0\right)=\frac{1}{2} \log \frac{1+\mu_{n, 0}}{1-\mu_{n, 0}} .
$$

Since

$$
\int_{\mathbb{D}}\left|\phi_{n}\right| d x d y=\int_{\mathbb{H}^{2}}\left\|\Phi_{n}\right\| d v_{n}<\infty,
$$

we can apply the main inequality in [R-S] and conclude that

$$
\frac{1}{1+\mu_{n, 0}} \int_{\mathbb{H}^{2}}\left\|\Phi_{n}\right\| d v_{\mathbb{H}^{2}} \leq \int_{\mathbb{H}^{2}} \frac{1}{1+\left|\mu_{n}\right|}\left\|\Phi_{n}\right\| d v_{\mathbb{H}^{2}}
$$

where $\mu_{n}$ is the complex dilatation of the quasi-conformal harmonic diffeomorphism with Hopf differential $\Phi_{n}$. Let $1>\delta>0$ and $k>1$ be fixed 
numbers. Define

$$
\begin{aligned}
& D_{n}=\left\{z \in \mathbb{H}^{2} \mid\left\|\Phi_{n}\right\|(z) \geq k\left\|\Phi_{n}\right\|_{B Q D}^{\frac{3}{4}}\right\}, \\
& U_{n}=\left\{z \in \mathbb{H}^{2} \mid\left\|\Phi_{n}\right\|(z)<k\left\|\Phi_{n}\right\|_{B Q D}^{\frac{3}{4}}\right\} .
\end{aligned}
$$

We have

$$
\int_{\mathbb{H}^{2}} \frac{1}{1+\left|\mu_{n}\right|}\left\|\Phi_{n}\right\| d v_{\mathbb{H}^{2}}=\left(\int_{D_{n}}+\int_{U_{n}}\right) \frac{1}{1+\left|\mu_{n}\right|}\left\|\Phi_{n}\right\| d v_{\mathbb{H}^{2}} .
$$

By Proposition 2.1, for any $z \in D_{n}$, the maximal $\Phi_{n}$-radius $R_{n, z}$ satisfies $R_{n, z} \geq C_{1} k$ for some absolute constant $C_{1}>0$. By the result on page 63 of [Hn], we have $\left|\mu_{n}\right| \geq \eta(k)$ for some constant $\eta(k)$ such that $\eta(k) \rightarrow 1$ as $k \rightarrow \infty$. Hence we have

$$
\int_{D_{n}} \frac{1}{1+\left|\mu_{n}\right|}\left\|\Phi_{n}\right\| d v_{\mathbb{H}^{2}} \leq \frac{1}{1+\eta(k)} \int_{D_{n}}\left\|\Phi_{n}\right\| d v_{\mathbb{H}^{2}}
$$

For any $0<\delta<1$, there exists $n_{0}$ such that if $n \geq n_{0}$ then

$$
\int_{U_{n}}\left\|\Phi_{n}\right\| d v_{\mathbb{H}^{2}} \leq \delta \int_{\mathbb{H}^{2}}\left\|\Phi_{n}\right\| d v_{\mathbb{H}^{2}}
$$

Combine this with (5.1)-(5.3), we have for $n \geq n_{0}$,

$$
\frac{1}{1+\mu_{n, 0}} \int_{\mathbb{H}^{2}}\left\|\Phi_{n}\right\| d v_{\mathbb{H}^{2}} \leq \frac{1}{1+\eta(k)} \int_{D_{n}}\left\|\Phi_{n}\right\| d v_{\mathbb{H}^{2}}+\delta \int_{\mathbb{H}^{2}}\left\|\Phi_{n}\right\| d v_{\mathbb{H}^{2}}
$$

Hence

$$
\frac{1}{1+\mu_{n, 0}} \leq \frac{1}{1+\eta(k)}+\delta
$$

Let $k \rightarrow \infty$, and then let $\delta \rightarrow 0$, we have

$$
\limsup _{n \rightarrow \infty} \frac{1}{1+\mu_{n, 0}} \leq \frac{1}{2}
$$

Since $0 \leq \mu_{n, 0}<1$ for all $n$, we have $\lim _{n \rightarrow \infty} \mu_{n, 0}=1$. From this, it is easy to see that $d_{\mathcal{T}}\left(\mathfrak{B}\left(\Phi_{n}\right), 0\right) \rightarrow \infty$.

Corollary 5.1. Let $\mathcal{F}$ be as in Proposition 5.1. Let $\Phi_{n}=\phi_{n} d z^{2} \in \mathcal{F}$. Suppose $\left\|\left|\Phi_{n}\right|\right\|=1$. Let $A_{n}=\int_{\mathbb{H}^{2}}\left\|\Phi_{n}\right\| d v_{\mathbb{H}^{2}}=\int_{\mathbb{D}}\left|\phi_{n}\right| d x d y$. Suppose $\lim _{n \rightarrow \infty} \phi_{n} / A_{n}=\psi$ such that $\int_{\mathbb{D}}|\psi| d x d y=1$, then $\mathfrak{B}\left(t_{n} \Phi_{n}\right) \rightarrow \infty$ for any $t_{n} \rightarrow \infty$. In particular $\mathfrak{B}$ is proper on any finite dimensional subspace of $\mathcal{F}$. 
Proof. For any $1>\delta>0$, we can find $1>r_{0}>0$, such that

$$
\int_{\mathbb{D}_{r_{0}}}|\psi| d x d y=1-\delta
$$

where $\mathbb{D}_{r_{0}}=\left\{|z|<r_{0}\right\}$. We have

$$
\int_{\mathbb{D}_{r_{0}}} \frac{\left|\phi_{n}\right|}{A_{n}} \geq 1-2 \delta
$$

provided $n$ is large enough. For any $k>0$, let $\epsilon_{n}=k t_{n}^{-\frac{1}{4}}$, where $t_{n} \rightarrow \infty$. Let

$$
U_{n}=\left\{z \in \mathbb{H}^{2}\left|\left\|t_{n} \Phi_{n}\right\|(z) \leq k\right| \| t_{n} \Phi_{n}||^{\frac{3}{4}}\right\}=\left\{z \in \mathbb{H}^{2} \mid\left\|\Phi_{n}\right\|(z) \leq \epsilon_{n}\right\} .
$$

Note that if $\Phi_{n}=\phi_{n} d z^{2}$, then for $z \in U_{n} \cap \mathbb{D}_{r_{0}}$

$$
\left|\phi_{n}\right|(z) \leq C_{1}\left(1-r_{0}\right)^{-2}|| \Phi_{n} \|(z) \leq \epsilon_{n}\left(1-r_{0}\right)^{-2}
$$

for some absolute constant $C_{1}$. Hence by (5.4),

$$
\begin{aligned}
\int_{U_{n}}\left\|\Phi_{n}\right\| d v_{\mathbb{H}^{2}} & \leq \int_{U_{n} \cap \mathbb{D}_{r_{0}}}\left|\phi_{n}\right| d x d y+\int_{\mathbb{D} \mid \mathbb{D}_{r_{0}}}\left|\phi_{n}\right| d x d y \\
& \leq \frac{\pi C_{1} \epsilon_{n}}{\left(1-r_{0}\right)^{2}}+2 \delta A_{n} .
\end{aligned}
$$

Since $\|\left|\Phi_{n}\right|||=1$, by applying the mean value inequality to $\left|\phi_{n}\right|(z)$ at a point $z_{n}$ with $\left\|\Phi_{n}\right\|\left(z_{n}\right) \geq \frac{1}{2}$, we conclude that $A_{n} \geq C_{2}$ for some abolute constant $C_{2}>0$. By the definition of $\epsilon_{n}$, we have $\epsilon_{n} \rightarrow 0$ as $n \rightarrow \infty$. Hence (5.5) implies that we have

$$
\begin{aligned}
\limsup _{n \rightarrow \infty} \frac{\int_{U_{n}}\left\|t_{n} \Phi_{n}\right\| d v_{\mathbb{H}^{2}}}{\int_{\mathbb{H}^{2}} \| t_{n} \Phi_{n} \mid d v_{\mathbb{H}^{2}}} & =\underset{n \rightarrow \infty}{\limsup } \frac{\int_{U_{n}}\left\|\Phi_{n}\right\| d v_{\mathbb{H}^{2}}}{A_{n}} \\
& \leq 2 \delta .
\end{aligned}
$$

Since $\delta$ is arbitrary, $t_{n} \Phi_{n}$ satisfies the conditions in the Proposition 5.2. Hence the first part of corollary is proved.

To prove the second part of the corollary, let $\mathcal{H}$ be a finite dimensional subspace of $\mathcal{F}$ with basis $\Psi_{1}, \ldots, \Psi_{k}$. If $\Phi_{n} \in \mathcal{H}$ is such that $\left\|\mid \Phi_{n}\right\| \rightarrow \infty$, then $\Phi_{n}=t_{n} \tilde{\Phi}_{n}$ such that $\left\|\tilde{\Phi}_{n}\right\| \|=1$ and $\tilde{\Phi}_{n}=\sum_{j=1}^{k} a_{n, j} \Psi_{j}$ with $\sum_{j=1}^{k} a_{n, j}^{2}$ being uniformly bounded. From this, it is easy to see that the second part of the corollary follows. 
Let $\Phi=\phi d z^{2} \in \mathcal{F}$. Define

$$
|\Phi|_{\infty}=\inf _{a \in \mathbb{D}} \sup _{z \in \mathbb{D}}|\phi(z)| \frac{|1-\bar{a} z|^{4}}{\left(\left.|1-| a\right|^{2}\right)^{2}}=\inf _{a \in \mathbb{D}} \sup _{\zeta \in \mathbb{D}}\left|\tilde{\phi}_{a}(\zeta)\right|,
$$

where $\tilde{\phi}_{a} d \zeta^{2}=\Phi$ and $\zeta=\frac{z-a}{1-\bar{a} z}$.

$$
|\Phi|_{L^{1}}=\int_{\mathbb{D}}|\phi| d x d y=\int_{\mathbb{H}^{2}}\|\Phi\| d v_{H^{2}} .
$$

Note that

$$
\int_{\mathbb{D}}|\phi| d x d y=\int_{\mathbb{D}}\left|\tilde{\phi}_{a}\right| d x d y
$$

if $\phi$ and $\tilde{\phi}_{a}$ are related as above.

Corollary 5.2. Let $\mathcal{F}_{1}=\left\{\left.\Phi \in B Q D|\||\Phi|\|=1,| \Phi\right|_{\infty}<\infty\right\}$. Let $\Phi_{n} \in \mathcal{F}_{1}$, and $t_{n} \rightarrow \infty$ be a sequence such that $\left|\Phi_{n}\right|_{\infty}\left|\Phi_{n}\right|_{L^{1}}^{-2}=o\left(t_{n}^{\frac{1}{4}}\right)$, then $d_{\mathcal{T}}\left(\mathfrak{B}\left(t_{n} \Phi_{n}\right), 0\right) \rightarrow \infty$. In particular, if $\left|\Phi_{n}\right|_{\infty}\left|\Phi_{n}\right|_{L^{1}}^{-2} \leq C$ for some constant $C$ independent of $n$, then $d_{\mathcal{T}}\left(\mathfrak{B}\left(t_{n} \Phi_{n}\right), 0\right) \rightarrow \infty$, for any $t_{n} \rightarrow \infty$.

Proof. For each $n$, by the definition, by a linear fractional transformation of $\mathbb{D}$ if necessary, we may assume that $\Phi_{n}=\phi_{n} d z^{2}$, with $\left(\sup _{z \in \mathbb{D}}\left|\phi_{n}\right|(z)\right)\left|\phi_{n}\right|_{L^{1}}^{-2}=$ $o\left(t_{n}^{\frac{1}{4}}\right)$. Let $M_{n}=\sup _{z \in \mathbb{D}}\left|\phi_{n}\right|(z)$ and $I_{n}=\left|\phi_{n}\right|_{L^{1}}$. We claim that

$$
I_{n}^{2} \leq C_{1} M_{n}
$$

for all $n$ and for some absolute constant $C_{1}$. Fix $n$, take $r_{0}$ such that

$$
\int_{\mathbb{D}\left(r_{0}\right)}\left|\phi_{n}\right| d x d y=\frac{1}{2} I_{n}
$$

where $\mathbb{D}\left(r_{0}\right)=\left\{z|| z \mid<r_{0}\right\}$. Since $\left\|\left|\Phi_{n} \|\right|=1\right.$, we have

$$
\frac{1}{2} I_{n}=\int_{\mathbb{D}\left(r_{0}\right)}\left|\phi_{n}\right| d x d y \leq C_{2} \int_{0}^{r_{0}}(1-r)^{-2} \leq \frac{C_{2}}{1-r_{0}}
$$

where $C_{2}$ is an absolute constant. On the other hand

$$
\frac{1}{2} I_{n}=\int_{\mathbb{D} \backslash \mathbb{D}\left(r_{0}\right)}\left|\phi_{n}\right| d x d y \leq M_{n} \cdot 2 \pi\left(1-r_{0}\right) .
$$


Hence

$$
\begin{aligned}
\frac{1}{2} I_{n} & \leq \frac{C_{2}}{1-r_{0}} \\
& \leq \frac{2 \pi M_{n} C_{2}}{\frac{1}{2} I_{n}} .
\end{aligned}
$$

From this (5.6) follows.

Now for any $k>0$, let

$$
\begin{aligned}
U_{n} & =\left\{z \in \mathbb{H}^{2}\left|\left\|t_{n} \Phi_{n}\right\|(z) \leq k\left\||| t_{n} \Phi_{n}\right\| \|^{3 / 4}\right\}\right. \\
& =\left\{z \in \mathbb{H}^{2}\left|\left\|\Phi_{n}\right\|(z) \leq k \epsilon_{n}\left\|\mid \Phi_{n}\right\| \|^{3 / 4}\right\}\right.
\end{aligned}
$$

where $\epsilon_{n}=t_{n}^{-\frac{1}{4}}$. Let $\delta_{n}=\left(\frac{k \epsilon_{n}}{M_{n} I_{n}^{-2}}\right)^{\frac{1}{2}}$. By (5.6) and the fact that $\epsilon_{n} \rightarrow 0$, we have $\delta_{n} \rightarrow 0$ as $n \rightarrow \infty$. As in the proof of Corollary 5.1, $I_{n} \geq C_{3}$ for some absolute constant $C_{3}$. Hence $\delta_{n} I_{n}^{-1} \rightarrow 0$ as $n \rightarrow \infty$. If $n$ is large enough so that $\delta_{n} I_{n}^{-1}<1$, then we take $r_{n}$ such that $1-r_{n}=\delta I_{n}^{-1}$. Denote $\mathbb{D}\left(r_{n}\right)=\left\{z|| z \mid<r_{n}\right\}$. Then

$$
\begin{aligned}
\int_{\left(\mathbb{D} \backslash \mathbb{D}_{n}\right) \cap U_{n}}\left|\phi_{n}\right| d x d y & \leq 2 \pi M_{n}\left(1-r_{n}^{2}\right) \\
& \leq 4 \pi\left(M_{n} I_{n}^{-2}\right) \delta_{n} I_{n} .
\end{aligned}
$$

On the other hand, as in the proof of (5.7), we have

$$
\begin{aligned}
\int_{\mathbb{D}\left(r_{n}\right) \cap U_{n}}\left|\phi_{n}\right| d x d y & \leq C_{4} k \epsilon_{n}\left(1-r_{n}\right)^{-1} \\
& =C_{4} k \epsilon_{n} \delta_{n}^{-1} I_{n}
\end{aligned}
$$

for some constant $C_{4}$ independent of $n$. Hence

$$
\begin{aligned}
\int_{U_{n}}\left|\phi_{n}\right| d x d y & \leq C_{5}\left\{M_{n} I_{n}^{-2} \delta_{n}+k \epsilon_{n} \delta_{n}^{-1}\right\} I_{n} \\
& =2 C_{5}\left\{k \epsilon_{n} M_{n} I_{n}^{-2}\right\}^{\frac{1}{2}} I_{n}
\end{aligned}
$$

for some constant $C_{5}$ independent of $n$. Hence if $n$ is large enough,

$$
\frac{\int_{U_{n}}\left|\phi_{n}\right| d x d y}{\int_{\mathbb{D}}\left|\phi_{n}\right| d x d y} \leq 2 C_{5}\left\{k \epsilon_{n} M_{n} I_{n}^{-2}\right\}^{\frac{1}{2}}
$$

By the assumption, the right side of the above inequality tends to zero as $n \rightarrow 0$ and the corollary follows. 
Example 1. Let $\Phi_{n}=\phi_{n} d z^{2}=c_{n} n^{2} z^{n} d z^{2}$, where $c_{n}$ is chosen so that $\left\|\left|\Phi_{n} \|\right|=1\right.$. Direct computations show that $C^{-1} \leq c_{n} \leq C$ for some positive constant $C$ independent of $n$. Then $\sup _{z \in \mathbb{D}}\left|\phi_{n}\right|(z)=c_{n} n^{2}$, and $\int_{\mathbb{D}}\left|\phi_{n}\right| d x d y=\frac{2 \pi n^{2} c_{n}}{n+2}$. Hence the $\left|\Phi_{n}\right|_{\infty}\left|\Phi_{n}\right|_{L_{1}}^{-2} \leq C^{\prime}$ for some constant $C^{\prime}$ independent of $n$. Hence by Corollary 5.2, for any subsequence $n_{k}$ and for any $t_{k} \rightarrow \infty$ we have $d_{\mathcal{T}}\left(\mathfrak{B}\left(t_{k} \Phi_{n_{k}}\right), 0\right) \rightarrow \infty$.

Example 2. Consider $(z-1)^{n} d z^{2}$. Then direct computation shows

$$
\sup _{z \in \mathbb{D}}(1-|z|)^{2}|z-1|^{n}=c_{n} n^{-2} 2^{n}
$$

where $C^{-1} \leq c_{n} \leq C$ for some constant $C>0$ independent of $n$. Let $\Phi_{n}=$ $c_{n}^{\prime} n^{2} 2^{-n}(z-1)^{n} d z^{2}=\phi_{n}(z) d z^{2}$, where $c_{n}^{\prime}$ is chosen so that $\left\|\left|\Phi_{n} \|\right|=1\right.$. Note that $\tilde{C}^{-1} \leq c_{n}^{\prime} \leq \tilde{C}$ for some $\tilde{C}>0$ independent of $n$. Let $a=-\frac{n+8-4 \sqrt{n+4}}{n}$, note that $-1<a<0$.

$$
\begin{aligned}
\sup _{z \in \mathbb{D}}\left|\phi_{n}(z)\right| \frac{|1-a z|^{4}}{\left(1-a^{2}\right)^{2}} & =c_{n}^{\prime} n^{2} 2^{-n} \sup _{0 \leq \theta \leq 2 \pi}\left|e^{i \theta}-1\right|^{n}\left|1-a e^{i \theta}\right|^{4}\left(1-a^{2}\right)^{-2} \\
& =c_{n}^{\prime} n^{2} \sup _{0 \leq \theta \leq 2 \pi} \sin ^{n} \frac{\theta}{2}\left[(1-a)^{2}+4 a \sin ^{2} \frac{\theta}{2}\right]^{2}\left(1-a^{2}\right)^{-2} \\
& =c_{n}^{\prime} n^{2} \sup _{0 \leq t \leq 1} t^{n}\left[(1-a)^{2}+4 a t^{2}\right]^{2}\left(1-a^{2}\right)^{-2}
\end{aligned}
$$

Let $f(t)=t^{n}\left[(1-a)^{2}+4 a t^{2}\right]^{2}$, then $f(0)=0$. Suppose $f(t), 0 \leq t \leq 1$ attains its maximum at $t_{0} \in(0,1)$, then $f^{\prime}\left(t_{0}\right)=0$, and

$$
n t_{0}^{n-1}\left[(1-a)^{2}+4 a t_{0}^{2}\right]^{2}+t_{0}^{n} \cdot 2\left[(1-a)^{2}+4 a t_{0}^{2}\right] \cdot 8 a t_{0}=0 .
$$

Hence $t_{0}^{2}=-\frac{n\left(1-a^{2}\right)}{4 a(n+4)}=1$ by the choice of $a$, which is impossible. Hence, for $0 \leq t \leq 1, f(t)$ attains its maximum at $t=1$. By (5.9), we have

$$
\begin{aligned}
\left|\Phi_{n}\right|_{\infty} & \leq c_{n}^{\prime} n^{2}\left[(1-a)^{2}+4 a\right]^{2}\left(1-a^{2}\right)^{-2} \\
& =c_{n}^{\prime} n^{2}(1+a)^{2}(1-a)^{-2} .
\end{aligned}
$$

Since $a<0,1-a>1$. Also

$$
1+a=1-\frac{n+8-4 \sqrt{n+4}}{n}=\frac{-8+4 \sqrt{n+4}}{n} .
$$


Hence

$$
\left|\Phi_{n}\right|_{\infty} \leq C_{1} n
$$

for some constant $C_{1}$ independent of $n$. On the other hand

$$
\begin{aligned}
\int_{\mathbb{D}}|z-1|^{n} d x d y & =\int_{2 \pi}^{0} \int_{0}^{1}\left|r^{2}+1-2 r \cos \theta\right|^{\frac{n}{2}} r d r d \theta \\
& =\int_{-\pi}^{\pi} \int_{0}^{1}\left|r^{2}+1+2 r \cos \theta\right|^{\frac{n}{2}} r d r d \theta \\
& \geq \int_{0}^{\frac{1}{\sqrt{n}}} \int_{0}^{1}(r+1)^{n}\left|1-\frac{r \theta^{2}}{(1+r)^{2}}\right|^{\frac{n}{2}} r d r d \theta \\
& \geq \frac{C_{2}}{\sqrt{n}} \int_{0}^{1}(r+1)^{n}\left(1-\frac{n}{2} \frac{r}{n(1+r)^{2}}\right) r d r \\
& \geq C_{3} 2^{n} n^{-\frac{3}{2}} \\
& \geq C_{4} n^{\frac{1}{2}}
\end{aligned}
$$

for some constants $C_{2}-C_{4}$ independent of $n$. By Corollary 5.2, we also have $\mathfrak{B}\left(t_{k} \Phi_{n_{k}}\right) \rightarrow \infty$ for all subsequence $n_{k}$ and for all $t_{k} \rightarrow \infty$.

In the last section of [Wn], it was proved that $\mathfrak{B}$ is continuous, and in section 4 of [T-W2], it was proved that the image of $\mathfrak{B}$ is open and $\mathfrak{B}$ is a diffeomorphism from $\mathrm{BQD}\left(\mathbb{H}^{2}\right)$ into $\mathcal{T}$. From the proof of the proposition 14 in [Wn], $\mathfrak{B}$ is in fact uniformly continuous on bounded subsets of $\mathrm{BQD}\left(\mathbb{H}^{2}\right)$. On the other hand, we have the following:

Proposition 5.3. Let $R>0$ and let

$$
B(R)=\left\{\Phi \in \mathrm{BQD}\left(\mathbb{H}^{2}\right)|||| \Phi \mid \| \leq R\right\} .
$$

Let $\mathcal{T}(R)=\mathfrak{B}(B(R))$. Then $\mathfrak{B}^{-1}$ is uniformly continuous on $\mathcal{T}(R)$.

Proof. For any complex measurable function $\mu$ on $\mathbb{D}$ such that $\|\mu\|_{\infty}<1$, denote $F^{\mu}$ to be the unique quasi-conformal map on $\mathbb{D}$ with boundary value $f^{\mu}$ which fixes $1, i,-i$. Suppose $f^{\mu}$ can be extended to a quasi-conformal harmonic diffeomorphism, then the harmonic map will be denoted by $\tilde{F}^{\mu}$ and its complex dilatation is denoted by $\tilde{\mu}$. By theorem 13 in [Wn], there exists $0<k<1$ such that if $[f] \in \mathcal{T}(R)$ then $\|\mu\|_{\infty} \leq k$, where $\mu$ is the 
complex dilatation of an extremal quasi-confomal map with boundary value in $[f]$.

$$
\mathcal{T}^{*}(R)=\left\{\mu \mid \mu \text { is measurable, }\|\mu\|_{\infty} \leq k \text { and }\left[f^{\mu}\right] \in \mathcal{T}(R)\right\} .
$$

Note that if $\mu \in \mathcal{T}^{*}(R)$, then $f^{\mu}$ can be extended to a quasi-conformal harmonic diffeomorphism with Hopf differential in $B(R)$. We claim that for any $\epsilon>0$, there is $\delta>0$ such that if $\mu, \nu$ in $\mathcal{T}^{*}(R)$ and $\|\mu-\nu\|_{\infty} \leq \delta$ then $\|\tilde{\mu}-\tilde{\nu}\|_{\infty} \leq \epsilon$. If the claim is true, then by the definition of $d_{\mathcal{T}}$ and by the method as in the proof of proposition 14 in [Wn], one can conclude that $\mathfrak{B}^{-1}$ is uniformly continuous on $\mathcal{T}(R)$.

First we prove the following, given $\epsilon>0$, there is $\delta>0$ such that if $\mu$ and $\nu$ are in $\mathcal{T}^{*}(R)$, then $|\tilde{\mu}(0)-\tilde{\nu}(0)| \leq \epsilon$. Suppose not, then there is $\epsilon>0$ and two sequences $\mu_{n}, \nu_{n}$ in $\mathcal{T}^{*}(R)$ such that $\left\|\mu_{n}-\nu_{n}\right\|_{\infty} \rightarrow 0$, but $\left|\tilde{\mu}_{n}(0)-\tilde{\nu}_{n}(0)\right| \geq \epsilon$. Since $\left\|\tilde{\mu}_{n}\right\|_{\infty} \leq k_{1}$ and $\left\|\tilde{\nu}_{n}\right\|_{\infty} \leq k_{1}$ for some $0<$ $k_{1}<1$ by [Wn], passing to subsequences if necessary, $\tilde{F}^{\mu_{n}}$ and $\tilde{F}^{\nu_{n}}$ converge uniformly on $\overline{\mathbb{D}}$ to normalized quasi-conformal harmonic diffeomorphisms $H_{1}$ and $H_{2}$ respectively. Since $\left\|\mu_{n}-\nu_{n}\right\|_{\infty} \rightarrow 0$ and $\mu_{n}, \nu_{n}$ are in $\mathcal{T}^{*}(R)$, $H_{1}$ and $H_{2}$ must have the same boundary value and so $H_{1}=H_{2}$ by [L-T3]. It then follows that $\left|\tilde{\mu}_{n}(0)-\tilde{\nu}_{n}(0)\right| \rightarrow 0$, which is a contradiction.

Now, for any $\epsilon>0$, let $\delta>0$ be as above. Let $\mu, \nu$ be in $\mathcal{T}^{*}(R)$ such that $\|\mu-\nu\|_{\infty} \leq \delta$. Let $a \in \mathbb{D}$ and $\phi(z)=(z-a) /(1-\bar{a} z)$. Define $\mu_{1}$ and $\nu_{1}$ by

$$
\mu_{1}(\phi(z))=\mu(z)\left(\frac{\phi^{\prime}(z)}{\left|\phi^{\prime}(z)\right|}\right)^{2}
$$

and

$$
\nu_{1}(\phi(z))=\nu(z)\left(\frac{\phi^{\prime}(z)}{\left|\phi^{\prime}(z)\right|}\right)^{2} .
$$

Then $f^{\mu_{1}}=h^{\mu} \circ f^{\mu} \circ \phi^{-1}$, and $f^{\nu_{1}}=h^{\nu} \circ f^{\nu} \circ \phi^{-1}$ where $h^{\mu}$ and $h^{\nu}$ are the linear fractional transformations which map $\mathbb{D}$ onto itself and are chosen so that $f^{\mu_{1}}$ and $f^{\nu_{1}}$ fix $1, i,-i$ respectively. Obviously $f^{\mu_{1}}$ and $f^{\nu_{1}}$ have quasi-conformal representatives $\tilde{F}^{\mu_{1}}$ and $\tilde{F}^{\nu_{1}}$. In fact,

$$
\tilde{F}^{\mu_{1}}=h^{\mu} \circ \tilde{F}^{\mu} \circ \phi^{-1}
$$

and

$$
\tilde{F}^{\nu_{1}}=h^{\mu} \circ \tilde{F}^{\nu} \circ \phi^{-1} .
$$

Moreover, the Hopf differentials of $\tilde{F}^{\mu_{1}}$ and $\tilde{F}^{\nu_{1}}$ are in $B(R)$. Hence $\mu_{1}, \nu_{1}$ are in $\mathcal{T}^{*}(R)$ becasue $\left\|\tilde{\mu}_{1}\right\|_{\infty}=\|\mu\|_{\infty} \leq k$ and $\left\|\tilde{\nu}_{1}\right\|_{\infty}=\|\nu\|_{\infty} \leq k$. 
We also have

$$
\tilde{\mu}_{1}(\phi(z))=\tilde{\mu}(z)\left(\frac{\phi^{\prime}(z)}{\left|\phi^{\prime}(z)\right|}\right)^{2}
$$

and

$$
\tilde{\nu}_{1}(\phi(z))=\tilde{\nu}(z)\left(\frac{\phi^{\prime}(z)}{\left|\phi^{\prime}(z)\right|}\right)^{2}
$$

Note that

$$
\left\|\mu_{1}-\nu_{1}\right\|_{\infty}=\|\mu-\nu\|_{\infty} \leq \delta
$$

Therefore

$$
|\mu(a)-\nu(a)|=\left|\mu_{1}(0)-\nu_{1}(0)\right| \leq \epsilon .
$$

Since $a$ is any point in $\mathbb{D}$, the claim follows.

\section{Appendix: Trajectories and Image Accumulation.}

In this appendix, seven figures of horizontal trajectories of holomorphic quadratic differentials are shown. These pictures of trajectories are produced by programming in Mathematica. Some trajectories may be broken due to slow convergence of the algorithm. In fact it should be smoothly defined for all time. Nevertheless, the qualitative behavior of the trajectory patterns is shown clearly. In some figures, the correponding harmonic map produces an image which has a good accumulation structure on the boundary. This structure is also shown on the unit disk. 


\section{Finitely Many Accumulations.}

Figure 1. $\Phi=e^{z} d z^{2}$.

(See Theorem 3.1 and Corollary 3.1.)

This example is the basis of all others. The trajectories have a $2 \pi i$ periodicity. The image of the corresponding harmonic map has an accumulation point at -1 .

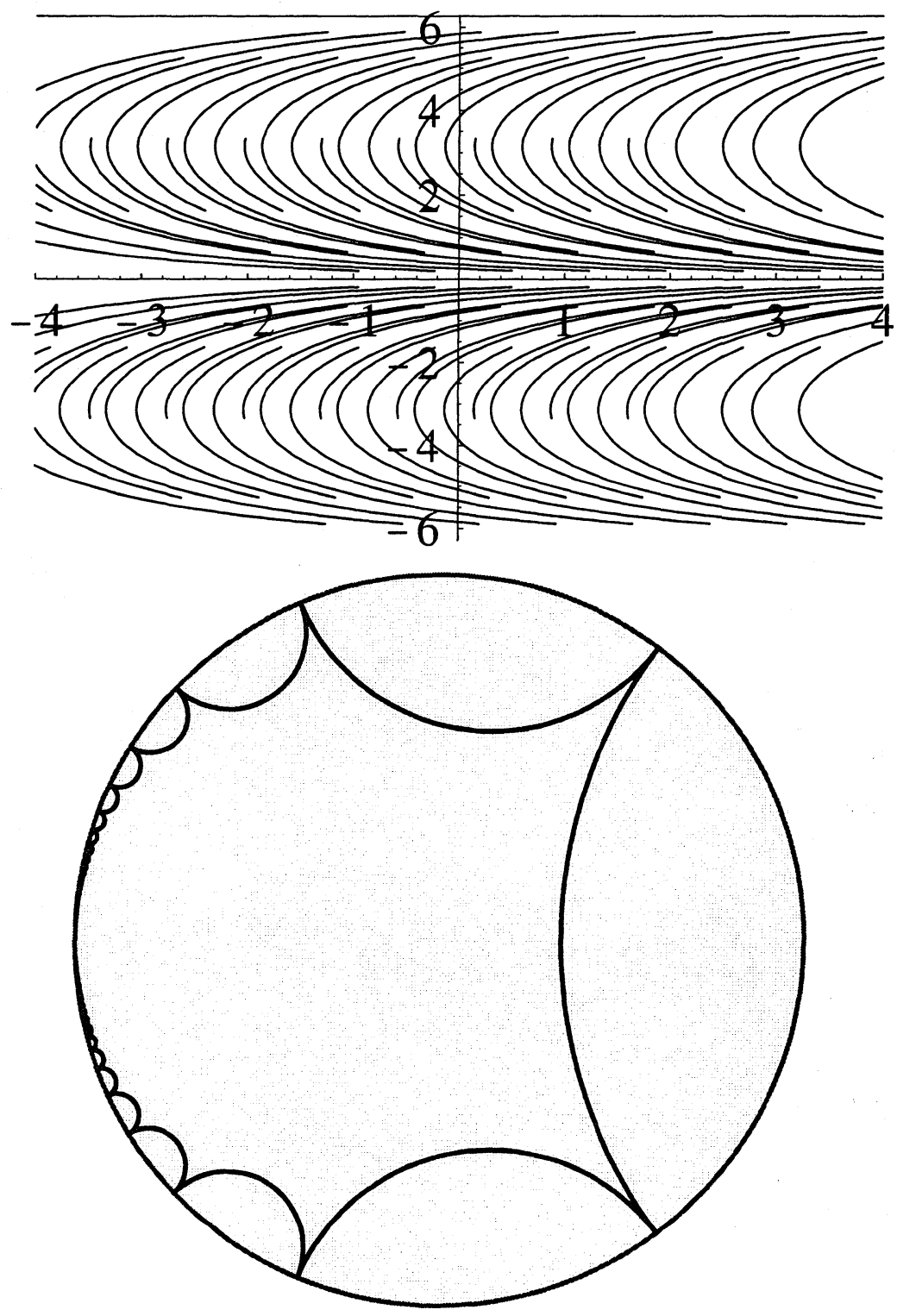


Figure 2. $\Phi=\left(e^{z}+1\right) d z^{2}$. (See Theorem 4.1.)

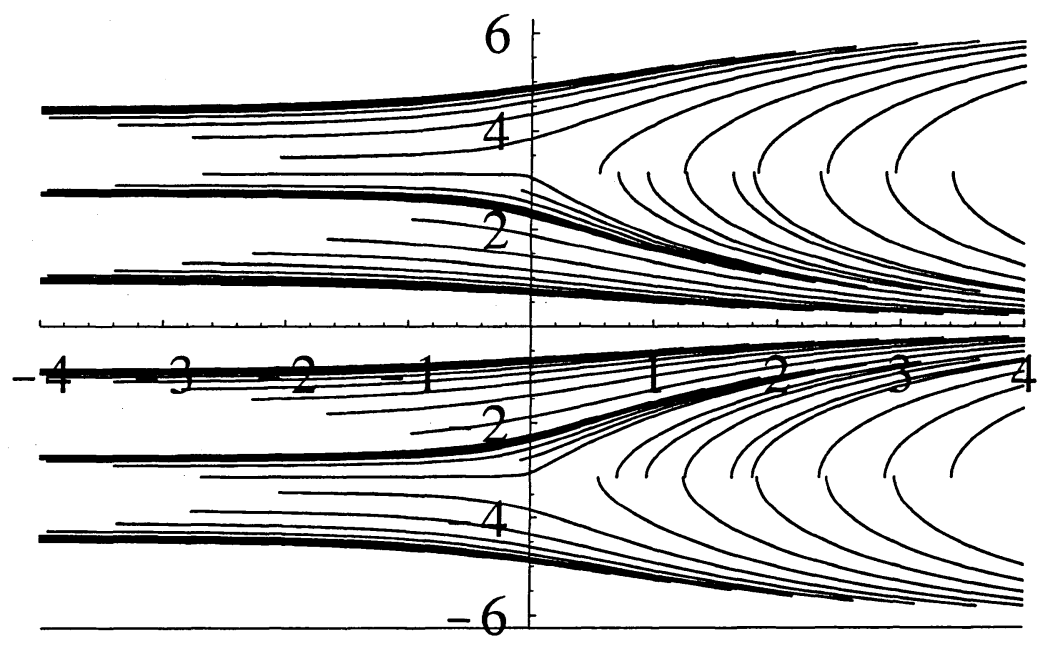


Figure 3. $\Phi=\left(e^{z}-1\right) d z^{2}$.

(See Theorem 4.1.)

From this and the previous one, a lower order term may significantly change the behavior of the harmonic map. This one has two accumulation points at \pm 1 while the previous one has only one.
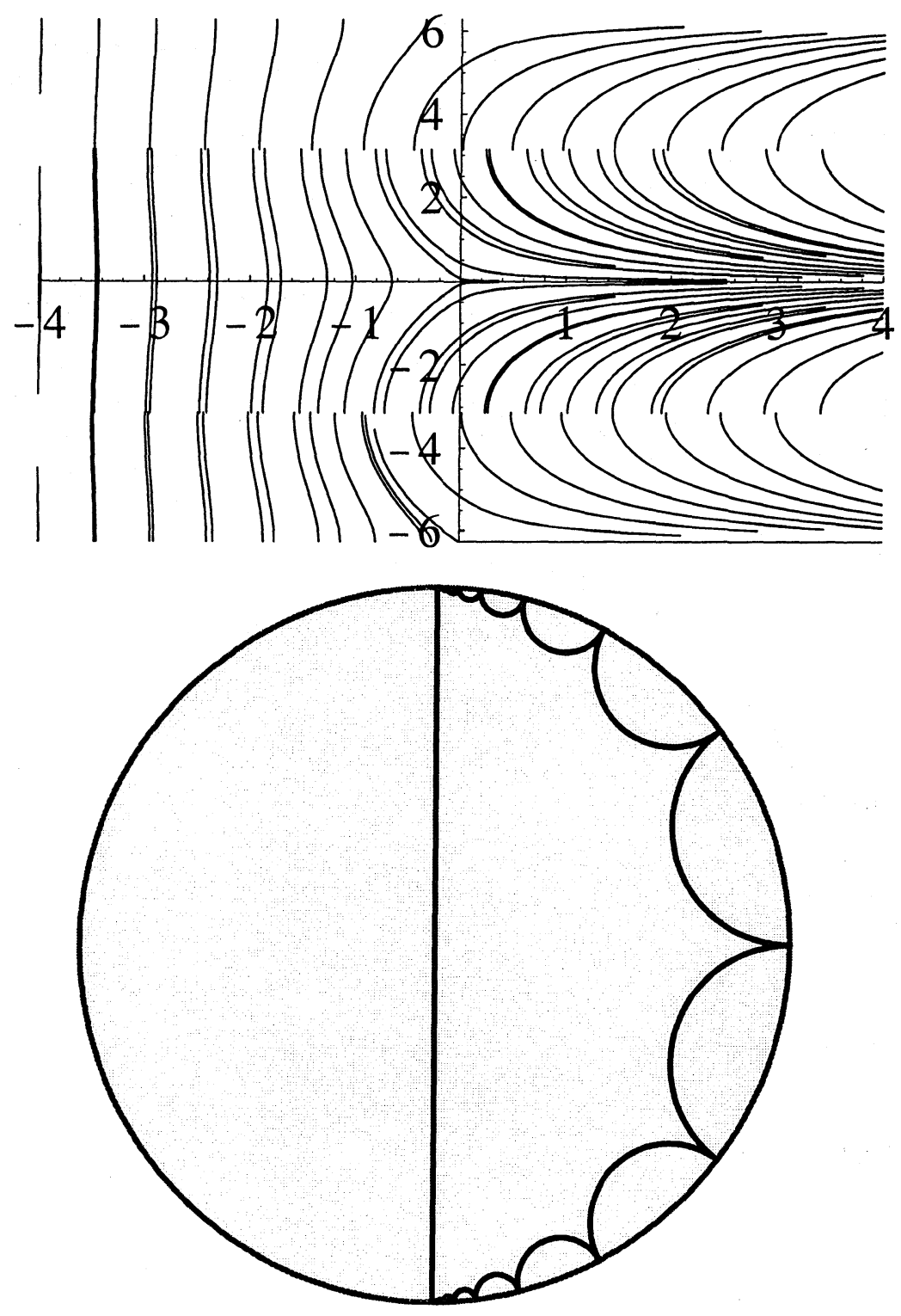
568

T.K.K. Au, L.-F. Tam \& T.Y.H. Wan

Figure 4. $\Phi=\sinh ^{2} z d z^{2}$.

(See Theorem 4.1)

This is another example that the fundamental region is different while there are also two accumulation points.
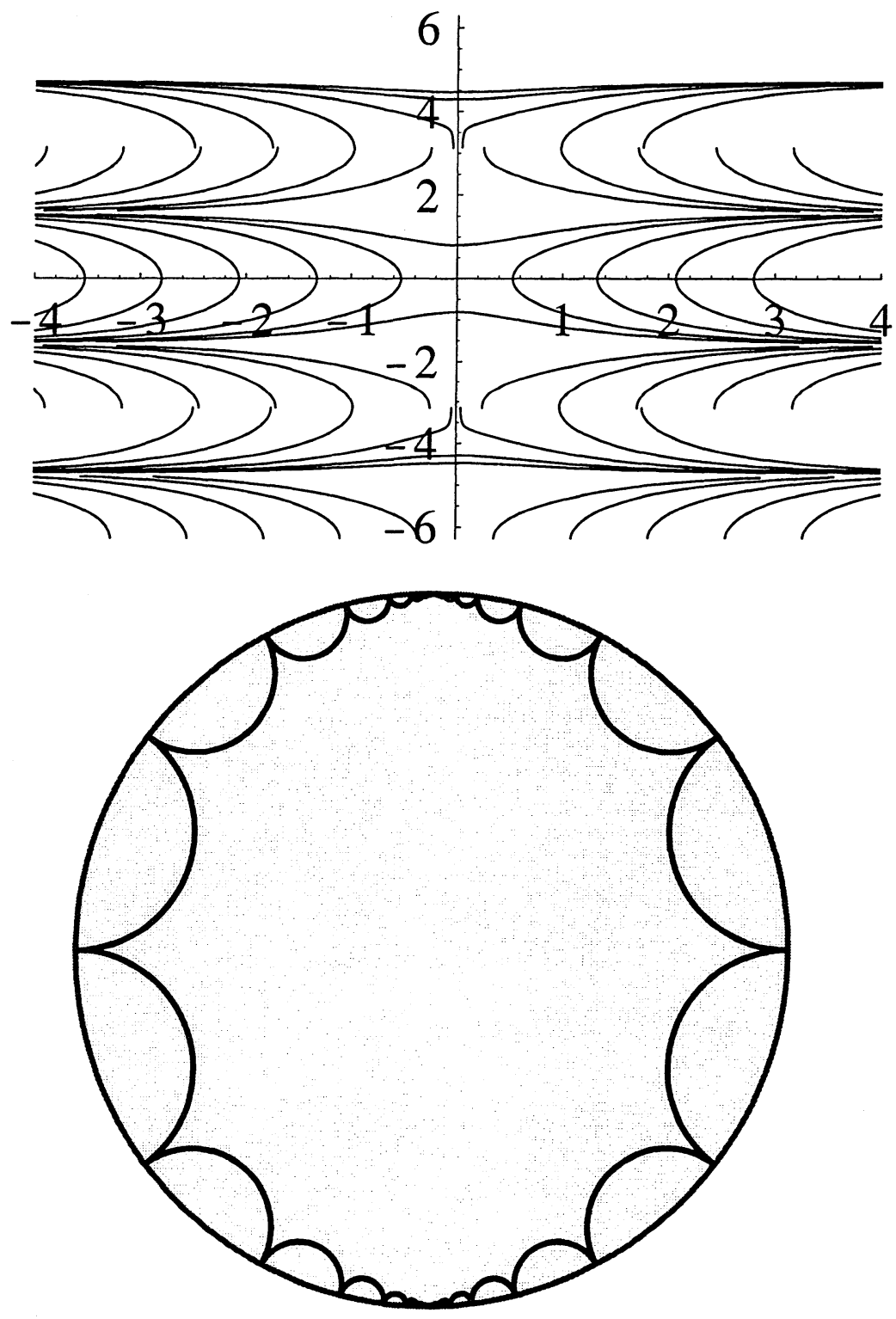
Figure 5. $\Phi=\left(z^{2}-1\right) e^{z^{2}} d z^{2}$.

(See Theorem 3.1 and Corollary 3.1.)

The image of this example should have 2 accumulation points.

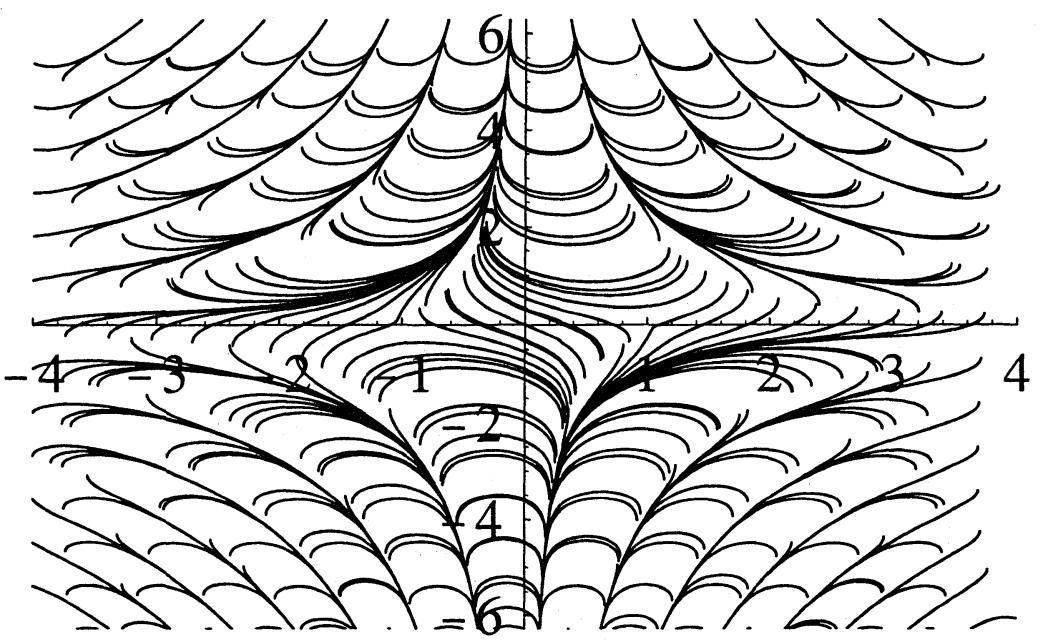

Figure 6. $\Phi=e^{z^{3}+z^{2}} d z^{2}$.

(See Theorem 3.1 and Corollary 3.1.)

The image of this example should have 3 accumulation points.

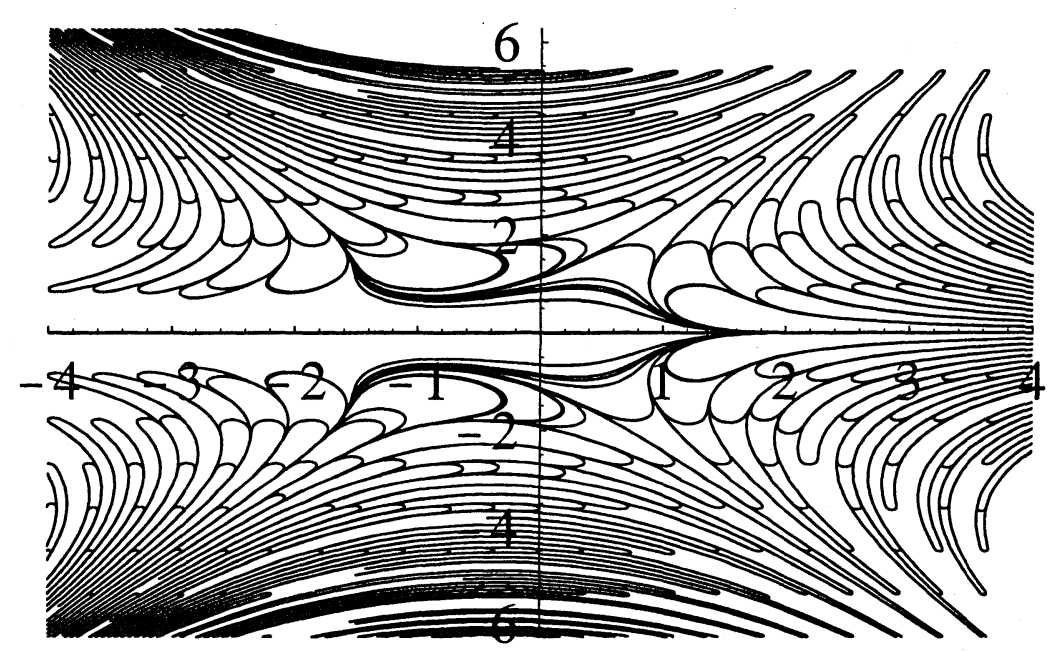




\section{Accumulation of accumulating points.}

Figure 7. $\Phi=e^{e^{z}} d z^{2}$.

(See Theorem 4.2)

Finally, this is an example about accumulation of accumulations. The image has infinitely many accumulation points marked by dots outside the unit circle, which in turns accumulate at -1 .

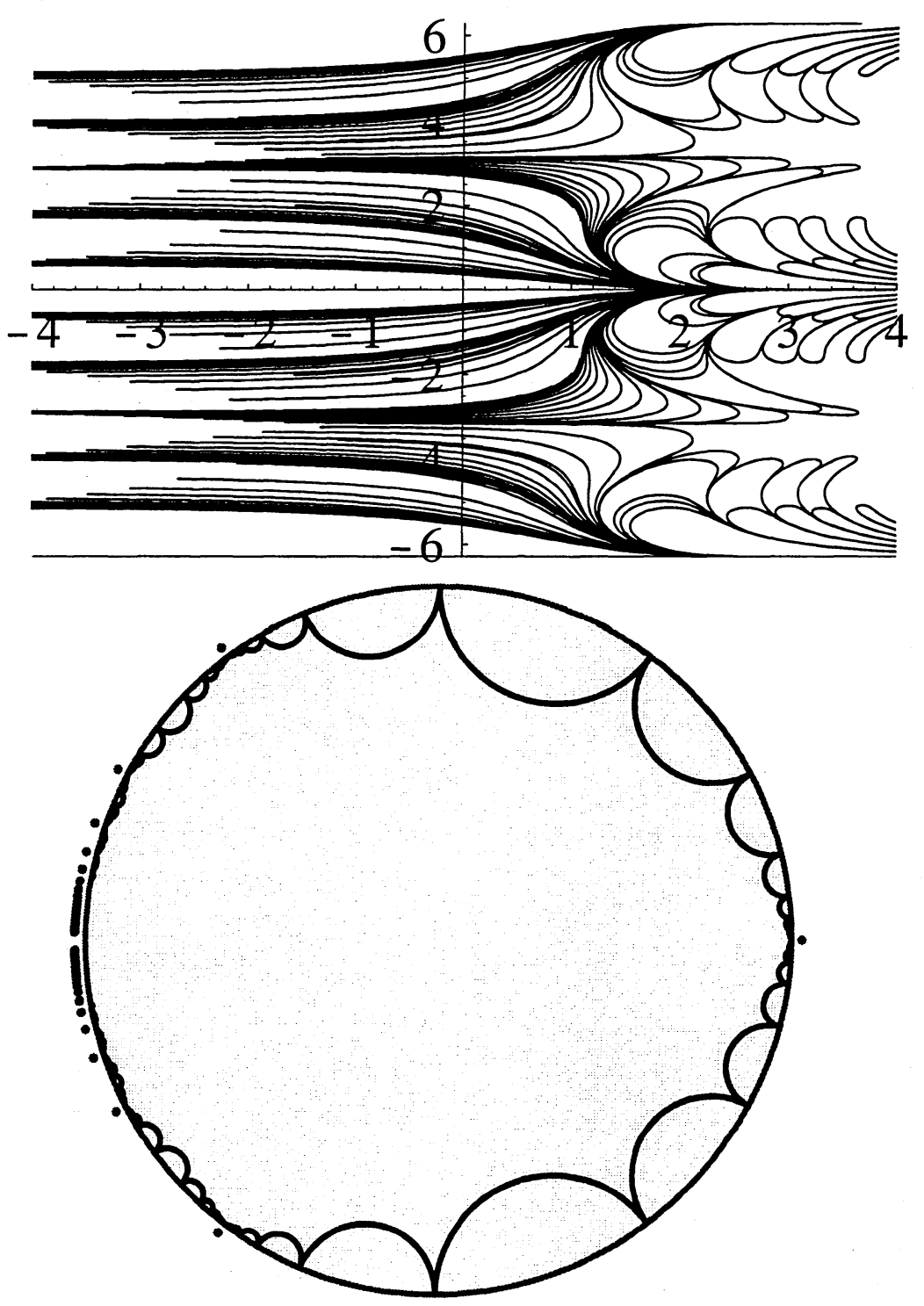




\section{References.}

[Ah] L.V. Ahlfors, Lectures on quasiconformal mapings, Van Nostrand, 1966.

[Ak] K. Akutagawa, Harmonic diffeomorphisms of the hyperbolic plane, Trans. Amer. Math. Soc., 342 (1994), 325-342.

[A-M-M] I. Anić, V. Marković, and M. Mateljević, Uniformly bounded maximal $\phi$-disks, Bers space and harmonic maps, Proc. Amer. Math. Soc., 128 (2000), 2947-2956; article electronically published on April 7, 2000.

[B] R.P. Boas, Entire functions, Academic Press, 1954.

[B-O] R.L. Bishop and B. O'Neill, Manifolds of negative curvature, Trans. Amer. Math. Soc., 145 (1969), 1-49.

[C-G] S.S. Chern and S. Goldberg, On the volume-decreasing property of a class of real harmonic mappings, Amer. J. Math., 97 (1975), 133-147.

[C-T] H.I. Choi and A. Treibergs, Gauss map of spacelike constant mean curvature hypersurface of Minkowski Space, J. Differential Geom., 32 (1990), 775-817.

[D-E] A. Douady and C.J. Earle, Conformally natural extension of homeomorphisms of the circle, Acta Math., 157 (1986), 23-48.

[Hn] Z.C. Han, Some Remarks Concerning the Geometric Behavior of Harmonic Maps between Surfaces, in 'Elliptic and Parabolic Methods in Geometry,' Ed. B.Chow, R. Gulliver, S. Levy, \& J. Sullivan, 1996, A K Peters, (1994), 57-66.

[HTTW] Z. Han, L.F. Tam, A. Treibergs, and T. Wan, Harmonic maps from the complex plane into surfaces with nonpositive curvature, Comm. Anal. Geom., 3 (1995), 85-114.

[H-W] R. Hardt and M. Wolf, Harmonic extensions of quasiconformal maps to hyperbolic space, Indiana U. Math., 46 (1997), 155-163.

[Hz] E. Heinz, Über die Losungen der Minimalflächengleichung, Nach. Akad. Wiss., Göttingen Math. Phys. Kl. II (1952), 51-56. 
[L-T1] P. Li and L.F. Tam, The heat equation and harmonic maps of complete manifolds, Invent. Math., 105 (1991), 1-46.

[L-T2] P. Li and L.F. Tam, Uniqueness and regularity of proper harmonic maps, Ann. Math., 136 (1992), 169-203.

[L-T3] P. Li and L.F. Tam, Uniqueness and regularity of proper harmonic maps II, Indiana U. Math. J., 42 (1993), 593-635.

[L-W1] P. Li and J.-P. Wang, Convex hull properties of harmonic maps, J. Differential Geom., 48 (1998), 497-530.

[L-W2] P. Li and J.-P. Wang, Harmonic rough isometries into Hadamard space, Asian J. Math., (1998), 419-442.

[L-W3] P. Li and J.-P. Wang, Counting massive sets and dimensions of harmonic functions, J. Differential Geom., 53 (1999), 237-278.

[M-M] V. Marković and M. Mateljević, New versions of Grötzsch principle and Reich-Strebel inequality, in '4th Symposium on Mathematical Analysis and Its Applications, Arandelovac, 1997,' Mat. Vesnik, 49 (1997), 235-239.

[M] Y. Minsky, Harmonic maps, length, and energy in Teichmüller space, J. Differential Geom., 35 (1992), 151-217.

[P] Ch. Pommerenke, Boundary behaviour of conformal maps, Springer-Verlag, 1992.

[R-S] E. Reich and K. Strebel, On quasiconformal mappings which keep the boundary points fixed, Trans. Amer. Math. Soc., 136 (1969), 211-222.

[S] R. Schoen, The role of harmonic mappings in rigidity and deformation problems, in 'Collection: Complex Geometry (Osaka, 1990),' Lecture Notes in Pure and Applied Mathematics, 143, Dekker, New York, (1993), 179-200.

[S-Y] R. Schoen \& S.-T. Yau, On Univalent Harmonic Maps between Surfaces, Invent. Math., 44 (1978), 265-278.

[S-T-W] Y.-G. Shi, L.-F. Tam, and T.Y.-H. Wan, Harmonic Maps on Hyperbolic Spaces with Singular Boundary Value, J. Differential Geom., 51 (1999), 551-600. 
[T-W1] L.F. Tam and T.Y.-H. Wan, Harmonic diffeomorphisms into Cartan-Hadamard surfaces with prescribed Hopf differentials, Comm. Anal. Geom., 2 (1995), 593-625.f

[T-W2] L.F. Tam and T.Y.-H. Wan, Quasi-conformal harmonic diffeomorphism and the universal Teichmüller space, J. Differential Geom., 42 (1995), 368-410.

[T-W3] L.F. Tam and T.Y.-H. Wan, On quasiconformal harmonic maps, Pacific J. Math., 182 (1998), 359-383.

[V] W.A. Veech, Second course in complex analysis, W. A. Benjamin, Inc., 1967.

[Wn] T.Y.-H. Wan, Constant mean curvature surface, harmonic maps and universal Teichmüller space, J. Differential Geom., 35 (1991), 643-657.

[W-A] T.Y.-H. Wan and T. Au, Parabolic constant mean curvature spacelike surfaces, Proc. Amer. Math. Soc., 120 (1992), 559-564.

[Wf] M. Wolf, High energy degeneration of harmonic maps between surfaces and rays in Teichmüller space, Topology, 30 (1991), 517540 .

[Y] D. Yang, Deforming a map into a harmonic map, preprint.

DEPARTMENT OF Mathematics

The Chinese University of Hong Kong

Shatin, Hong Kong

E-mail address: thomasaumath.cuhk.edu.hk

E-mail address: Iftammath.cuhk.edu.hk

E-mail address: tomwanmath.cuhk.edu.hk

ReCeived June 15, 2000. 